\title{
AN ARBITRARY LAGRANGIAN-EULERIAN FINITE ELEMENT METHOD FOR LARGE DEFORMATION ANALYSIS OF ELASTIC-VISCOPLASTIC SOLIDS
}

\author{
Somnath GHOSH \\ Department of Engineering Mechanics, The University of Alabama, Tuscaloosa, AL 35487, USA \\ Noboru KIKUCHI \\ Department of Mechanical Engineering and Applied Mechanics, The University of Michigan, \\ Ann Arbor, MI 48109, USA
}

Received 5 Jily 1988

Revised manuscript received 17 August 1989

\begin{abstract}
Analysis of large deformation of elastic-viscoplastic materials has been performed in this paper using the finite element method with the arbitrary Lagrangian-Eulerian description. An overstress type viscoplastic model using the internal variable approach in a rotated stress-strain space characterizes the material. Stable and efficient integration techniques for the viscoplastic relations are discussed. A linearized form in the ALE description is presented which is to be solved using iteration techniques. In particular the quasi-Newton methods have been used in this analysis. Several test problems which have been considered illustrate the effectiveness of the entire solution algorithm.
\end{abstract}

\section{Introduction}

Traditionally speaking, finite deformation problems in solid mechanics have been solved using a Lagrangian description for the finite element mesh. In this description, the finite element mesh is embedded in, and moves with the material constituting the continuum. The pure Lagrangian approach has the advantage of having to satisfy less complex governing equations, compared to the pure Eulerian approach. This may be attributed to the absence of the convection terms, and also simple updating techniques for path and history dependent materials in the former approach. However, a significant limitation of this description is encountered when the solid deformation becomes large. Lack of control over the mesh movement results in distorted (sometimes entangled) meshes with large changes in element dimensions, which adversely affects the accuracy of the solution. Secondly, problems involving certain contact boundaries, especially those with sharp edges or corners, may not be represented precisely in this description. This is due to the fact that the boundary condition has to be specified on a material point which might move itself. Situations of this kind are frequently encountered in the numerical simulation of metal forming processes, e.g. extrusion, drawing etc., where the punch or die faces may have acute edges or abrupt surface discontinuities. Despite the introduction of sophisticated remeshing schemes [1] to circumvent the problems associated with excessive mesh distortion in the Lagrangian description, an 
accurate contact boundary manifestation is often questionable. Also, in most cases, remeshing after every time step becomes very expensive. Resorting to a pure Eulerian approach in which the mesh remains stationary, on the other hand, introduces other difficulties like appropriate representation of the free boundary. In spite of its capability to represent internal deformation effectively, the Eulerian mesh is less suited for domains whose boundaries or interfaces move substantially. The shortcomings of each of the above descriptions encountered in large deformation analysis, calls for a description which can combine the advantages of both the above approaches into a single description. Such a description, in which the finite element mesh need not adhere to the material but may be in general motion relative to the material is known as the arbitrary Lagrangian-Eulerian description. The ALE description reserves the potential to represent a Lagrangian or Eulerian description as limiting cases at points, where such descriptions are desired. Thus, it is evident that an ALE description is ideally suited (sometimes necessary) for solving a variety of complex problems in solid mechanics, especially those dealing with large deformation metal forming and fracture.

The arbitrary Lagrangian-Eulerian description has been used in fluid mechanics [2] with a finite difference mesh and more recently in the finite element analysis of solid-fluid interaction problems $[3,4]$ for modeling the fluid. Argyris and his coworkers have also formulated a generalized Eulerian-Lagrangian description in the context of flow problems with NavierStokes equations in [5]. In their paper [5], they have successfully applied a moving reference frame to represent the finite element nodal points that model the fluid, which is interacting with a deformable solid undergoing unsteady motion. However, its use in solid mechanics problems has been restricted mainly because of the additional effort required in satisfying the equilibrium conditions and updating complexities. In the recent years, researchers like Haber [6], Benson [7], Liu et al. [8] have implemented the ALE techniques successfully to solve nonlinear problems in solid mechanics. However, all of the above authors have used explicit time integration schemes for handling the transient effects arising in large deformation problems with material nonlinearity. Explicit methods, though noted for their efficient performance with a class of structural problems, especially those with high frequency response such as in the case of impact and shock, suffer from the lack of generality of application. In general, the time steps in explicit methods are restricted by stringent stability conditions which require the evaluation of the maximum eigenvalue of the system. Frequently, this is rather inconvenient in nonlinear problems, as the eigenvalues change with the evolution of solution. Belytschko [9] has obtained bounds on the eigenvalues based on an evaluation of the Raleigh quotient and has stated that very often these upper bounds are drastically overestimated leading to much smaller time steps than are required for stability. Consequently, implicit methods, which have more flexibility with respect to numerical stability, are often more suited in the finite element analysis of inertial problems.

A vital observation is also made while modeling metal forming processes at elevated temperatures. Very often it is imperative to include viscoplastic (time-dependent plastic) material behavior in the material model when analyzing such processes. It has been noticed that problems involving materials with a relaxation time are most susceptible to numerical instabilities arising from mathematically stiff nonlinear differential equations. It seems natural that the stability conditions of the finite element initial value problem is governed by the stability behavior of the temporal discretization at the constitutive level. The issue of numerical stability for quasistatic elasto/viscoplastic problems has been addressed by Cor- 
meau [10] for the explicit Euler's method, from which he concludes that a stable solution is achieved only if the solution is within strict bounds derived for the particular constitutive models. Researchers like Kumar et al. [11] have also explored the feasibility of explicit schemes with time step control. However, as pointed out by Hughes and Taylor [12], the time steps required for numerical stability with explicit schemes are much smaller than those necessary for accuracy for slowly varying loads. In general, the bounds on the time step restrict the inelastic strain increments to be much less than the elastic strains. Since the elastic strains in the large deformation problems are infinitesimally small, this implies that the explicit schemes require a prohibitively large number of time steps to complete the finite strain analysis. To overcome this limitation of time step bounds, a variety of unconditionally stable implicit algorithms based on the generalized trapezoidal rule or midpoint rule, have been proposed in [12-14]. A number of these algorithms when examined carefully, as in [14], yield second order accurate solutions.

Mesh partitioning techniques which introduce implicit and explicit elements in separate parts of a domain to reduce the computing cost in comparison with the fully implicit mesh have been found to be suitable for certain problems in solid mechanics [15]. However, at the moment, such a partitioning does not look very promising with an arbitrary LagrangianEulerian mesh based on the fact that time integration is used to update variables associated with material points which may advect across the elements, thus making it difficult to define the zones a priori. The above argument does not hold for the fully implicit methods which maintain a uniformity over the entire domain in this respect.

A major drawback of the implicit method is the evaluation of the tangent iteration matrix during each iteration step performed for achieving dynamic equilibrium. However, the superlinearly convergent quasi-Newton methods have proven to be extremely beneficial in alleviating these obstacles associated with implicit algorithms. These methods replace the Jacobian matrix in Newton's method with an approximation matrix that is updated by mere matrix multiplication at each iteration step resulting in a drastic reduction in the computing effort. The explosive growth in computer technology at the level of supersystems and mini/micro computers have also had a tremendous impact in the sophistication of finite element codes. A large number of nonlinear computer codes, e.g. NIKE3D, ADINA, etc., have implemented implicit schemes because of their flexibility. Thus, in the perspective of the above discussion, an arbitrary Lagrangian-Eulerian finite element method with implicit time integration seems to possess attractive features which are desirable in a large deformation analysis of materials exhibiting inelastic behavior.

As indicated earlier, the main objective of this work is to develop a finite element model for large deformation analysis of elastic-viscoplastic materials. The response of materials subjected to loading at elevated temperatures $(\sim 0.5$ melting point $)$ are best represented by such rate dependent plastic models exhibiting the characteristics of combined creep and plasticity. Among the various unified models which include internal state variables to define the current state of deformation, the overstress models exhibiting rate dependence after a yield stress is reached have gained relative preference in the computational area. This may be attributed to the complex yield criterion in the theories that include rheologic effects prior to yielding. An overstress viscoplastic model with isotropic work hardening proposed by Perzyna [16] has been modified in this analysis, from macroscopic considerations, to include the effects of large deformation and initial anisotropy [17]. The latter has been incorporated with sheet metal 
forming in mind, where grain orientation due to pre-rolling gives rise to structure anisotropy. The above model has prospects of further modification to include temperature dependence, rate-dependent yield strength and plastic anisotropy, and a few of these will be addressed in a future paper.

From the previous discussion on time integration, it is apparent, that in any numerical scheme employed for the analysis of elasto-viscoplastic problems, integration of the rate constitutive equations to obtain the internal variables and stresses is of utmost importance. In particular, objective rate forms of stresses and internal variables have to be integrated to yield their updated values at the end of a time step in an incremental formulation. The conventional methods based on elasto-viscoplastic modulus suffer from various shortcomings especially in satisfying the plasticity consistency conditions, and very often these methods require elaborate schemes for making transition from elasticity to plasticity resulting in the truncation of time steps. In an attempt to overcome these limitations, various researchers have conveniently exploited the additive structure of the rate of deformation (strain rate in small deformation) tensor to introduce return mapping algorithms. These stem from the operator split of the complete set of spatial rate constitutive equations and the use of product formula techniques. The three major requirements of an integration algorithm, viz. consistency with the constitutive relations, numerical stability and incremental objectivity, can be satisfied in these algorithms with a careful choice of component algorithms. Several forms of the return mapping algorithms, e.g. the radial return method [18, 19], the closest point algorithm [20], the mean normal procedure [21] have been cited in literature. However, most of these algorithms are applicable to simple plasticity models consisting of associated plastic flow, simple yield criteria, flow rules, etc. Among these, the closest point algorithm is of relatively general applicability, but unfortunately it suffers from laborious evaluations of gradients of plastic flow direction, normal to the yield surface, plastic modulii, etc. for non-trivial plasticity. Recently Ortiz and Simo [22] have come up with a return mapping algorithm which is capable of handling a wide variety of plastic/viscoplastic models without having to go through the extensive computations of the closest point algorithms. Plastic state is attained at a quadratic rate of convergence with this algorithm. The central idea of this algorithm has been extended for applicability to the set of constitutive relations discussed above in this analysis. It involves stress relaxation based on subcycling, thus avoiding the necessity to iterate for the solutions.

In this paper, an ALE finite element model for large deformation analysis of rate sensitive materials has been presented. It starts with a brief review of the governing equation in the arbitrary Lagrangian-Eulerian description in Section 2. The constitutive relations for an elastic-viscoplastic material are discussed in Section 3. A corotational formulation using rotated components of the Cauchy stress tensor and its conjugate deformation rate is developed for its simplicity with anisotropic materials. Specific relations have been presented for initially transversly isotropic materials which are suitable for certain sheet metal forming analysis. In Section 4, the weak forms for the ALE description within the implicit time integration frame work are described. A return mapping scheme for integration of the viscoplastic rate equations is discussed in Section 5. Section 6 deals with the linearization techniques used and linearized version of the weak form. The numerical implementation techniques have been considered in detail in Section 7 and finally some numerical examples consolidating the validity of the model are presented in Section 8. 


\section{Governing field equations in the arbitrary Lagrangian-Eulerian description}

A detailed discussion on the derivation and meaning of governing field equations, viz. the continuity, momentum and energy equations in the arbitrary Lagrangian-Eulerian description, has been presented in an earlier paper [17] by the authors. In this paper, we shall briefly review the significance of these equations for the clarity of presentation.

The ALE formulation introduces a reference configuration which consists of a set of grid points in arbitrary motion in space. Each point of this reference configuration may be unambiguously identified by an invariant set of three independent coordinates $\chi_{i}$. The motion of the reference frame may then be expressed as an arbitrary continuous function of $\chi_{i}$ and the time $t$,

$$
x_{i}=x_{i}\left(x_{j}, t\right) .
$$

The formulation requires an inverse to (1) to exist, i.e.

$$
\chi_{i}=\chi_{i}\left(x_{j}, t\right),
$$

such that the Jacobian $J=\left|\partial x_{i} / \partial \chi_{j}\right|$ is non-vanishing. The above description of the referential system does not refer directly to the motion of the particles constituting the continuum and it is therefore necessary to establish a correlation between this system and the Lagrangian system which inherently defines the motion of the particles. Such a relation demands a unique connection between the two systems at every stage of deformation of the continuum, i.e., a single material point may coincide with only one point (not necessarily the same point always) of the grid system. In the Lagrangian system the material particles are identified by a set $c i$ coordinates $\boldsymbol{X}$ known as the material coordinates. In the deformed configuration the material particle may occupy a position $x=\left(x_{1}, x_{2}, x_{3}\right)$, which may be expressed in terms of the material coordinates and time $t$ as

$$
x_{i}=x_{i}\left(X_{j}, t\right),
$$

where the inverse of (3) is assumed to exist such that the determinant of the Jacobian $\hat{J}=\left|\partial x_{i} / \partial X_{j}\right|$ is not equal to zero. To obtain a relation between the referential and material coordinates, we assume a point $x$ in space occupied at a time $t$ by elements of both the systems (material point and grid point). Thus, by virtue of the existence of the inverse mappings of (1) and (3), an interrelation between the motion of material and referential (grid) points may be established as

$$
\chi_{i}=f_{i}\left(x_{j}, t\right)=\hat{f}_{i}\left(X_{k}, t\right) .
$$

Equation (4) may be interpreted as a mapping of the material domain onto the referential domain. With the notion that such a relation exists, it is then possible to derive the conservation equations of mechanics with respect to a referential configuration. The Reynolds transport theorem is applied to an arbitrary control volume moving through a deforming 
continuum which is then shrunk to a point (see [17] for details). This yields the resulting field equations in the arbitrary Lagrangian-Eulerian description as

Continuity:

$$
\left.\frac{\partial(\rho J)}{\partial t}\right|_{x}+J \frac{\partial}{\partial x_{i}}\left(\rho\left(V_{i}-W_{i}\right)\right)=0
$$

Momentum:

$$
\rho G_{i}+\frac{\partial \sigma_{j i}}{\partial x_{j}}=\left.\rho \frac{\partial V_{i}}{\partial t}\right|_{x}+\rho\left(V_{j}-W_{j}\right) \frac{\partial V_{i}}{\partial x_{j}}
$$

Energy:

$$
\left.\rho \frac{\partial \hat{u}}{\partial t}\right|_{x}+\rho\left(V_{j}-W_{j}\right) \frac{\partial \hat{u}}{\partial x_{j}}=\sigma_{j i} D_{j i}-\frac{\partial q_{i}}{\partial x_{i}}+f
$$

where $\rho$ refers to the material density, $\sigma_{i j}$ is the Cauchy stress tensor, $G$ is the body force vector per unit mass, $\hat{u}$ is the specific internal energy, $q$ is the heat flow vector, $D$ is the rate of deformation tensor, and $f$ is the rate of heat addition by a source. In the above equations $W\left(=\partial x /\left.\partial t\right|_{x}\right)$ represents the velocity of a representative grid point and $V\left(=\partial x /\left.\partial t\right|_{x}\right)$ represents the velocity of a material point coinciding with the grid point at time $t$.

The above set of equations (5)-(7) may be interpreted as material conservation laws with respect to arbitrarily moving grid points. A noteworthy advantage of the ALE description is that, in general, the velocity $W$ of the reference frame (representative grid point) may not be the same as the coincident material point velocity $V$ as in the Lagrangian description, or be zero as in the Eulerian description. In the event, that a grid point may coincide with a material point, the relative velocity term $(W-V)$ becomes zero, resulting in the vanishing of the convection terms and consequently the set of equations becomes Lagrangian. Similarly, a pure Eulerian description is obtained by simple setting $W=0$.

It is this flexibility to use a different description pointwise, where the grid points may be made to move with material or remain stationary in space or even to move with any arbitrary velocity, that makes the arbitrary Lagrangian-Eulerian description so attractive. This feature provides a greater scope for the user to maneuver the nodal or grid points effectively in a large deformation analysis.

When analyzing problems in solid mechanics, especially nonlinear materials with time and history dependent variables using the ALE description, it can be observed from (5)-(7) that a relationship between time derivatives, with material and referential coordinates fixed, is necessary. This relation may be obtained by direct comparison of the ALE conservation laws with the corresponding Lagrangian form $(W=V)$. If $\beta$ is a time-dependent variable for the material points, then the following relation may be established:

$$
\left.\frac{\partial \boldsymbol{\beta}}{\partial t}\right|_{x}=\left.\frac{\partial \boldsymbol{\beta}}{\partial t}\right|_{x}+\left(W_{k}-V_{k}\right) \frac{\partial \boldsymbol{\beta}}{\partial x_{k}} \text {. }
$$

This equation provides the link necessary for updating various material variables like velocity, 
density, temperature, Cauchy's stress, etc., to the grid points at the end of each time step in an ALE formulation. The above set of equations, together with the constitutive relations discussed in the next section form a basis for the weak forms used in the finite element formulation for large deformation analysis using the arbitrary Lagrangian-Eulerian description.

\section{Constitutive equations}

The constitutive relations for elastic-viscoplastic materials adopted in this paper are rate type, based on the additive decomposition of the rate of deformation into elastic and inelastic parts. This requires the choice of an appropriate objective stress rate, which depends on the class of materials being considered. In this analysis, a general formulation has been carried out for elastically transversely isotropic materials. It has been shown by Green and Naghdi [23] and subsequently by Green and McInnis [24], that for general anisotropic materials a constitutive relation together with a yield criterion is best represented in a rotated Lagrangian system. Their contention was further confirmed by Dienes [25] and Johnson and Bammann [26] who extended its use to solve successfully, problems of finite deformation with simple shear and kinematic hardening plasticity. In this description, the stress rate is derived from the material time derivative of a stress tensor (also noted as the rotated Cauchy stress tensor) transformed into a rotated space of the material deformation to yield

$$
\boldsymbol{t}=\boldsymbol{R}^{\mathrm{T}} \boldsymbol{\sigma} \boldsymbol{R},
$$

where $t$ is the rotated stress tensor, $\boldsymbol{\sigma}$ is the Cauchy stress tensor and $\boldsymbol{R}$ is the proper orthogonal tensor obtained from the polar decomposition of the deformation gradient tensor $\boldsymbol{R}\left(\boldsymbol{R}=\boldsymbol{F} \boldsymbol{U}^{-1}\right)$. The rotated stress tensor $t$, which has the same principal invariants as the Cauchy stress tensor, is the true stress which would result if the deformation gradient was the stretch $U$ alone. An interesting consequence of this transformation is that the conjugate strain rate for this stress is the rotated rate of deformation tensor $d,\left(d=R^{\mathrm{T}} D R\right.$, where $D$ is the rate of deformation tensor) which, when integrated in time, yields a physically meaningful strain measure. The frame invariant constitutive model is therefore formulated in a rotated stress-strain space.

In this model, it is assumed that the rotated rate of deformation tensor $\boldsymbol{d}$ admits an additive decomposition into elastic and inelastic parts. A thermodynamic argument for such decomposition is provided within the framework of internal variable theory, where such a decomposition may be obtained independent by any kinematic considerations. Thus

$$
d=d^{\mathrm{e}}+d^{\mathrm{vp}},
$$

where $d^{\mathrm{c}}$ represents the elastic part and $d^{\mathrm{vp}}$ represents the viscoplastic part of the deformation rate tensor. The material time derivative of rotated Cauchy stress may then be related to the rate of rotated deformation tensor through the elasticity relations as

$$
i=E:\left(d-d^{\mathrm{vp}}\right),
$$


where $E$ is a fourth order elasticity tensor which may be a function of the spatial stress tensor in general. The above equation is not valid for materials which exhibit hyperelastic response but is typical of hypoelastic materials which do not admit a stored energy.

In the characterization of the viscoplastic part of the rate of deformation, an internal state variable approach is adopted, where the response functions depend on the past history of the independent variables only through the present values of certain variables describing the internal state of the continuum. Within this approach; there exists two categories of models, viz. (i) theories that assume yield criteria which separate purely elastic deformations from combined elastic-viscoplastic deformations and (ii) theories that assume no yield criterion and allow the possible existence of elastic and viscoplastic deformations at all stages of loading. It should be noted that the power law model has been widely used by material scientists to simulate metals. However, a recent critical study by Eftis and Jones [27] indicates that the yield based theories in general offer a greater promise in modeling a wide variety of inelastic behavior like loading/unloading, rate and history effects, isotropic and kinematic hardening with Bauchinger effects, creep/relaxation, etc. A three-dimensional unified, overstress model of this category, described by Perzyna [16] has been appropriately extended to include the effects of large deformation and initial anisotropy. In this description, the rate of rotated viscoplastic deformation tensor is given as

$$
d_{i j}^{\mathrm{vp}}=\gamma\left\langle\Phi\left(\bar{t}-\kappa\left(W_{\mathrm{p}}, \theta\right)\right)\right\rangle \partial \bar{t} / \partial t_{i j},
$$

where $\gamma$ is a temperature dependent viscosity coefficient, \langle\rangle is the MacCaulay operator, $\kappa$ is an internal state variable, in particular a parameter describing isotropic work hardening, $W_{p}$ is the inelastic work and $\theta$ is the absolute temperature. The term $\bar{t}$ may be defined as an effective rotated Cauchy stress which may be written as

$$
\begin{gathered}
\bar{t}=\left(\frac { 1 } { 2 } \left[(F+G) t_{11}^{2}+(F+G) t_{22}^{2}+2 G t_{33}^{2}-2 F t_{11} t_{22}-2 G t_{22} t_{33}\right.\right. \\
\left.\left.-2 G t_{11} t_{33}+2(G+2 F) t_{12}^{2}+6 M t_{23}^{2}+6 M t_{31}^{2}\right]\right)^{1 / 2}
\end{gathered}
$$

for transversely isotropic materials. The scalars $F, G$ and $M$ are parameters of anisotropy which depend on the amount of inelastic strain accumulation and the temperature. Details of evaluating these parameters may be found in [17].

The rate of deformation tensor defined by (12) is for associated plastic flow and therefore satisfies the normality and incompressibility conditions. The viscoplastic model, though incorporates isotropic hardening, does not include kinematic hardening. Also, the model suffers from not having a rate-dependent yield criterion. The function $\Phi(F)$ may be chosen according to the material being modeled and the conditions of the experiment and various choices have been cited in [16]. It is interesting to note that the relations for inviscid plasticity may be derived as a limiting case of (12) in the event that the viscosity parameter $\gamma$ goes to infinity or when the motion is very rapid.

The set of constitutive relations (12) will be complete by supplementing them with the evolution laws for the internal variables. The rate-dependent characterization of plasticity will reside in the characterization of these evolution laws. The growth law used in this model assumes a linear relation between the rate of change of the work hardening parameter $\kappa$ and 
the equivalent rate of viscoplastic deformation tensor:

$$
\partial \kappa / \partial t=E_{\mathrm{p}} \bar{d}^{\mathrm{vp}}
$$

where $E_{\mathrm{p}}$ is an equivalent plastic modulus. Despite the fact that other nonlinear forms may be more realistic from a representation point of view, the evaluation of the anisotropic parameters are simplified with the linearity assumption as shown in [17]. Thus, the set of equations (11)-(14) form the basis of the material model used in this analysis.

\section{Weak forms and finite element approximations in the arbitrary Lagrangian-Eulerian description}

Weak forms of the mass and momentum equations may be obtained by taking the product of (5) and (6), respectively, with appropriate weighting functions and integrating over the current grid volume. However, in the finite element analysis of time-dependent finite deformation problems, emphasis is placed on the proper time integration scheme and hence the selection of the appropriate configuration. It is apparent from the discussion given in the introduction that an implicit time integration scheme is better suited for the class of inertial problems considered here. In this analysis, we have chosen to neglect the transient term $\left(\rho \partial V_{i} /\left.\partial t\right|_{x}\right)$ in the momentum balance equation (6), i.e., we have assumed a quasi-static process with respect to a grid point.

An overall stability analysis for implicit schemes used in the nonlinear initial value problem with an ALE description is extremely difficult because of the nonlinear, nonsymmetric convection operators together with the nonlinearities arising out of the material constitutive relations. The selection of the implicit method has therefore been based on a critical examination of the conclusions drawn from the stability analysis of a few associated systems.

Two notions of stability exist in general. One of them is the concept of linearized stability based on a linearization of the nonlinear system. Studies on the linearized stability criteria of one step time integration algorithms for linear and nonlinear heat conduction problems with symmetric operators have been carried out in [28-30]. These indicate that in the nonlinear regime, the generalized mid-point family of algorithms are unconditionally stable for a much wider range of the parameter $\alpha\left(\alpha \geqslant \frac{1}{2}\right)$ compared to the generalized trapezoidal family. However, this does not necessarily imply that a conservation law or decay inequality imposes limits upon the growth of the solutions. As a matter of fact, pathological energy growth have been noticed for unconditionally linearized stable algorithms for nonlinear problems. Consequently, a second notion of stability based on physically appropriate energy growth characteristics is now widely used in nonlinear analysis. Such a global energy criterion when applied to the above two integration schemes indicate more favorable conclusions for the midpoint and trapezoidal family of algorithms [30].

In problems of fluid mechanics, the convection term in an Eulerian description gives rise to an oscillatory solution when the Galerkin formulation is used. In the recent years various upwinding techniques like 'upstream weighting', 'Petrov-Galerkin finite element method' have been used to stabilize the solution. It is observed that the oscillatory behavior is apparent when the local Peclet number which is proportional to the velocity becomes large ( 2). In 
solid mechanics problems, such stabilizing may therefore be necessary when treating highly transient problems like impact, shock, etc. However, in the class of inertial problems that are of primary interest here, the difference between the grid and the material velocity is not believed to be large enough to initiate significant oscillations in the solution. Nevertheless, it is also reassuring to notice that an energy analysis of the algorithms mentioned above for certain convective systems with nonlinear terms guarantees unconditional stability for the generalized mid-point family [30].

In a numerical scheme for the solution of the boundary value problem for elasticviscoplastic materials, stability of the integration schemes for rate constitutive relations have a significant contribution to the overall stability of the system. In a recent discussion on the accuracy and stability characteristics of integration schemes, Ortiz and Popov [14] have pointed out that the general class of return mapping algorithms that have been used in this analysis are contained in the families of generalized trapezoidal and midpoint algorithms. Based on a linearized stability and accuracy analysis of the two families mentioned above for elasto-plastic constitutive relations, they have concluded that, while both of them render second order accuracy for a choice $\alpha=\frac{1}{2}$ of the algorithmic parameter, their response to stability is quite different. The stability properties of the generalized trapezoidal rule are very sensitive to the degree of distortion of the loading surface whereas the generalized midpoint rule is unconditionally stable for $\alpha \geqslant \frac{1}{2}$, regardless of the choice of the loading surface. This notable conclusion establishes the superiority of the midpoint family over the trapezoidal family for any arbitrary material model. All of the above studies on stability of numerical schemes for systems related to the one in question, point in the direction of an implicit finite element formulation utilizing the favorable conditions of the generalized midpoint rule. Specifically, from maximum accuracy considerations, the above discussion together with the contention that evaluation of displacement gradients at the midstep $\left(\alpha=\frac{1}{2}\right)$ gives no straining due to rigid body rotations [31] speak favorably in terms of a weak form at the midstep configuration.

Using the generalized midpoint rule, the principle of virtual work is written in an intermediate grid configuration $\Omega\left(t_{n+\alpha}\right)$ as

$$
\begin{gathered}
\int_{\Omega\left(t_{n+a}\right)} \sigma_{j i} \frac{\partial \bar{u}_{i}}{\partial x_{j}} \mathrm{~d} \Omega+\int_{\Omega\left(i_{n+a}\right)} \rho\left(V_{i}-W_{j}\right) \frac{\partial V_{i}}{\partial x_{j}} \bar{u}_{i} \mathrm{~d} \Omega \\
=\int_{\Omega\left(t_{n+a}\right)} \rho G_{i} \bar{u}_{i} \mathrm{~d} \Omega+\int_{\Gamma\left(t_{n+a}\right)} T_{i} \bar{u}_{i} \mathrm{~d} \Gamma,
\end{gathered}
$$

where all variables are at the mid-step configuration, corresponding to time $t_{n+a}$ with the position of a grid being interpolated as

$$
x^{n+\alpha}=(1-\alpha) x^{n}+\alpha x^{n+1} \text { for } 0 \leqslant \alpha \leqslant 1 \text {. }
$$

As mentioned in Section 2, an unambiguous connection is required between the Lagrangian and the referential system. This implies that the boundary of the grid domain never leaves the boundary of the solid or quantitatively

$$
(V-W) \cdot n=0,
$$


where $\boldsymbol{n}$ is the outward normal to the grid boundary. This enables the accommodation of all kinds of boundary conditions. The boundary of the grid is assumed to be the union of displacement, traction and contact types, i.e., $\Gamma(t)$ is $\Gamma_{i U}(t) \cup \Gamma_{i T}(t) \cup \Gamma_{i C}$ for $1 \leqslant i \leqslant 3$, with the usual respective conditions imposed. In special cases, such as boundaries with concentrated loads it might be necessary to make the corresponding load application points Lagrangian.

Obviously, the above weak form (15) demands the evaluation of the different variables in the intermediate equilibrium configuration occurring between two consecutive equilibrium states. Let $\boldsymbol{\beta}^{n}$ and $\boldsymbol{\beta}^{n+1}$ correspond to values of any such variable of the problem at time $t_{n}$ and $t_{n+1}$, respectively. The midstep value of this variable may then be obtained by a linear interpolation between its two end values as

$$
\boldsymbol{\beta}^{n+\alpha}=(1-\alpha) \boldsymbol{\beta}^{n}+\alpha \boldsymbol{\beta}^{n+1} .
$$

It is assumed that the value of the variable is known at a grid point in the $n$-th configuration. Its value in the $(n+1)$-th grid configuration may be calculated intough the evaluation of the grid point increment of the variable $\Delta^{\mathrm{g}} \boldsymbol{\beta}$, by using the generalized midpoint integration as

$$
\Delta^{\mathrm{g}} \boldsymbol{\beta}=\boldsymbol{\beta}^{n+1}-\boldsymbol{\beta}^{n}=\Delta t\left(\left.\frac{\partial \beta}{\partial t}\right|_{\chi}\right)^{n+\alpha} .
$$

However, for path-dependent material variables like Cauchy stress, velocity, work hardening parameter, etc., the evaluation procedure of the increment $\Delta^{g} \beta$ is not direct. For this type of variables, the grid point increment must be derived from the material point increment $\Delta^{\mathrm{m}} \boldsymbol{\beta}$. The relation between the time derivatives of a variable at a fixed material point and a grid point (8) may be numerically integrated to yield this desired relation as

$$
\Delta^{z} \beta=\Delta^{m} \beta+\Delta t\left(W_{k}^{n+\alpha}-V_{k}^{n+\alpha}\right) \frac{\partial \beta^{n+\alpha}}{\partial x_{k}^{n+\alpha}} .
$$

Figure 1 clearly shows the distinction between the two increments.

REMARK 1. The contact boundary is incorporated using the well-known exterior penalty formulation for unilateral contact problems. It should be emphasized that, though in the present work only normal contact with a rigid surface in the absence of friction is considered, an extension to the treatment of contact of two deformable surfaces is trivial. An extensive treatment on the penalty formulation of unilateral contact problems may be found in $[32,33]$.

The central idea of this formulation lies in the introduction of interface springs to preclude penetration. The possible contact boundary $\boldsymbol{\Gamma}_{\mathrm{C}}$ in this formulation is assumed to consist of purely Lagrangian nodes. Constructing a local coordinate system $\theta_{\xi}(1 \leqslant \xi \leqslant 3)$ on each part of the candidate contact boundary, a procedure similar to that discussed in $[32,33]$ may be adopted to reach the following non-penetration condition at the intermediate configuration:

$$
\psi^{n+\alpha}\left(\theta_{1}^{n}+\alpha \Delta u_{1}, \theta_{2}^{n}+\alpha \Delta u_{2}\right) \geqslant \theta_{3}^{n}+\alpha \Delta u_{3} .
$$



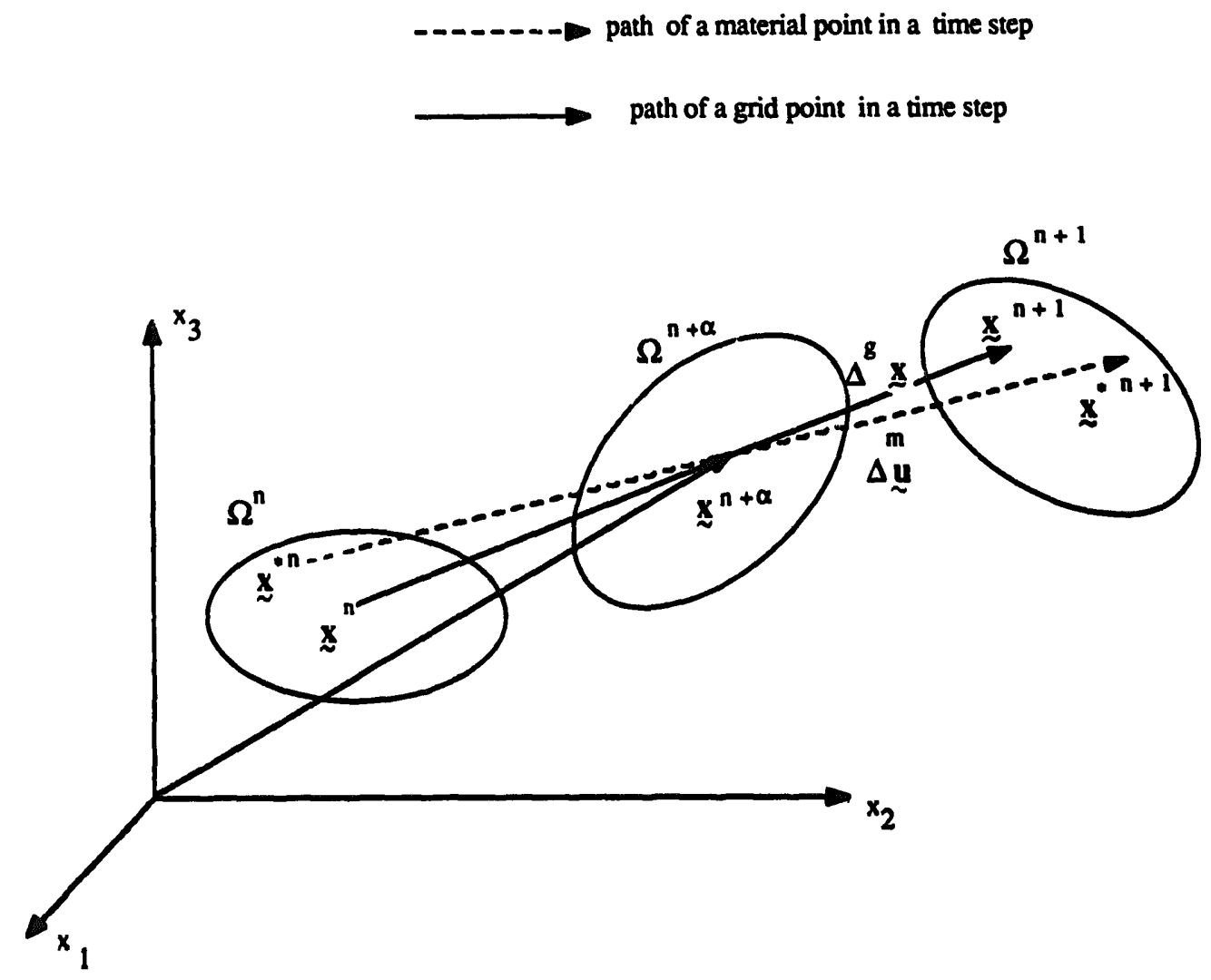

Fig. 1. Motion of a grid point and material point in a time step in the ALE description.

In (21) $\psi^{n+a}\left(\theta_{1}, \theta_{2}\right)$ is the parametric representation of the rigid surface at time $t_{n+a}$ and $\theta_{\xi}^{n}$ represents the coordinates of a point on the contact boundar at time $t_{n}$. It is assumed that the positive $\theta_{3}$ coordinate is roughly in the direction of the inward normal to the rigid surface. Linearization of the function $\psi$ in $\alpha \Delta u_{1}$ and $\alpha \Delta u_{2}$ results in the following contact condition:

$$
\alpha \Delta u \cdot n^{n+\alpha}-g \leqslant 0,
$$

where $n^{n+\alpha}$ is the inward normal to the rigid surface at the intermediate step and $g$ is the gap function defined in [32]. The constraint condition may then be incorporated in the principle of virtual work (15) through the use of an exterior penalty method to yield the approximate approximation for traction as

$$
T^{n+\alpha}=T^{n}+\alpha \Delta T_{n} n^{n+\alpha}=T^{n}+k_{n}\left\langle\alpha \Delta u_{n}-g\right\rangle n^{n+\alpha} \text { on } \Gamma_{C},
$$

where $\langle>$ is the MacCaulay operator. The above equation (23) is a result of the assumption that the contribution to traction within a time step, is due to normal contact alone. The penalty parameter $k_{n}(\rightarrow \infty)$ is representative of the rigidity of stiff normal springs on the contact boundary. It is evident from (23) that only in the case of contact an increment of pressure is activated. 
REMARK 2. The midstep value of the density is evaluated directly by solving the weak form of the continuity equation. This equation, after the application of the constraint condition (17) may be written as

$$
\left.\int_{\Omega\left(t_{n+\alpha}\right)} \frac{\partial \rho}{\partial t}\right|_{\chi} \bar{\rho} \mathrm{d} \Omega=\int_{\Omega\left(t_{n+\alpha}\right)} \rho\left(V_{j}-W_{j}\right) \frac{\partial \bar{\rho}}{\partial x_{j}} \mathrm{~d} \Omega-\int_{\Omega\left(t_{n+\alpha}\right)} \rho \frac{\partial W_{i}}{\partial x_{i}} \bar{\rho} \mathrm{d} \Omega .
$$

Equation (24) is solved for $\left(\partial \rho /\left.\partial t\right|_{x}\right)^{n+\alpha}$ from which density at the intermediate step may be obtained as

$$
\rho^{n+\alpha}=\rho^{n}+\alpha \Delta t\left(\frac{\partial \rho}{\partial t}\right)^{n+\alpha}
$$

The solution to the temperature problem may similarly be obtained easily by considering the weak form of the energy balance equation (7), but it has not been considered in the present work and will be presented in a forthcoming paper by the authors. The set of equations discussed above, must now be solved after finite element discretization using iterative techniques and these will be discussed in Section 7.

\section{Integration algorithm for rate type constitutive equations of elasto-viscoplasticity}

A well-known fact about the integration algorithms for rate type constitutive relations in large deformation, solid mechanics problems is that, they must necessarily satisfy three basic requirements, namely (a) consistency with the constitutive relations, (b) numerical stability and (c) incremental objectivity. The difficulties associated with the fulfillment of these requirements in the traditional tangent modulus method have given way to the more efficient return mapping alogithms which may be implemented for a wide variety of elastic-plastic/ viscoplastic constitutive models. These algorithms evolve as a consequence of the additive decomposition of the rate of deformation tensor into elastic and viscoplastic parts.

It was noticed by Ortiz et al. in [20] that the complete set of rate constitutive equations itself admits an additive decomposition, resulting from the decomposition of the spatial rate of deformation tensor mentioned above. Such a split of the set of evolution equations, also known as the 'operator split', calls for the use of product formula techniques to construct an efficient integration algorithm. The product formula suggest the independent treatment of the component operators, i.e., the result of an algorithm consistent with the first operator is operated on by an algorithm consistent with the second operator and so on. An attractive feature of the product algorithms is that if each of the component algorithms are numerically consistent and unconditionally stable, then the global algorithm is also consistent and unconditionally stable. In the present context, let $\boldsymbol{F}^{\mathrm{el}}(h)$ and $\boldsymbol{F}^{\mathrm{vp}}(h)$ be two unconditionally stable, elastic and viscoplastic algorithms, respectively, which are consistent with the set of constitutive equations for every time step $h>0$. Then, according to the above proposition, the resulting global algorithm $\boldsymbol{F}(h)=\boldsymbol{F}^{\mathrm{vp}}(h) \boldsymbol{F}^{\mathrm{el}}(h)$ is also unconditionally stable and consistent with the constitutive relations.

In this analysis, this idea of the product algorithm in conjunction with operator split of the 
set of evolutionary equations has been used. Let us first assemble the entire set of evolution equations for elasto-viscoplasticity from Section 3 as

$$
\begin{aligned}
& d=d^{\mathrm{e}}+d^{\mathrm{vp}}, \quad i=E: d^{\mathrm{e}}, \\
& d^{\mathrm{vp}}=\gamma\left\langle\Phi\left(\bar{t}-\kappa\left(W_{p}, \theta\right)\right)\right\rangle \frac{\partial \bar{t}}{\partial t}, \quad \frac{\partial \kappa}{\partial t}=E_{\mathrm{p}} \bar{d}^{\mathrm{vp}} .
\end{aligned}
$$

In the above set of equations the elasticity matrix, the viscosity coefficient and the plastic modulus are assumed to remain constant over the entire deformation process. The additive structure of the rate of deformation tensor introduces an additive decomposition of (26) into an elastic and a viscoplastic part:

(a) Elastic:

$$
\begin{aligned}
& d=d^{\mathrm{c}}+d^{\mathrm{vp}}, \quad \dot{t}=E: d, \\
& d^{\mathrm{vp}}=0, \quad \frac{\partial \kappa}{\partial t}=0 ;
\end{aligned}
$$

(b) Visco-plastic:

$$
\begin{aligned}
& d=d^{\mathrm{u}}+d^{\mathrm{vp}}=0, \quad i=-E: d^{\mathrm{vp},} \\
& d^{\mathrm{vp}}=\gamma\left\langle\Phi\left(\bar{t}-\kappa\left(W_{p}, \theta\right)\right)\right\rangle \frac{\partial \bar{t}}{\partial t}, \quad \frac{\partial \kappa}{\partial t}=E_{\mathrm{p}} \bar{d}^{\mathrm{vp} .} .
\end{aligned}
$$

It is to be observed that the right-hand sides of (27) and (28) add up to the right-hand side of (26) in accordance with the notion of operator split. The operator split of the entire set of equations (26) has an important significance. The set of elastic equations (27) indicates that the solid does not respond viscoplastically in this part, i.e., the viscoplastic strains and the work hardening parameter do not change. The response of the solid is thus entirely elastic. Contrarily, in the viscoplastic set of equations (28) the total strain rate is zero, i.e., the solid behavior is absolutely rigid. With this operator split, an applied product algorithm has the following implication. The elasto-viscoplastic is solved by first solving an elastic problem to obtain the deformation and elastic stress predictor at each time step. The viscoplastic algorithm then relaxes the stress predictors and updates the internal variables (work hardening parameter) so that viscoplastic consistency is achieved. Global consistency and unconditional stability may thus be attained if each of these algorithms are devised to possess these characteristics individually. We next discuss the elastic and plastic algorithms separately required for the global analysis.

Elastic algorithm. In the implicit integration algorithms applied to spatial rate constitutive equations, linear difference operators are often used on stresses for updating their values to the next consecutive configuration. In the implementation of the linear space operators, care should be taken to represent the relation between tensorial quantities with respect to the same basis vectors, i.e., the tensor fields should be associated with a common configuration. It has been demonstrated by Pinsky et al. [34] that the rotated stress formulations may be candidates 
to such implicit algorithms with an approximation. If it is assumed that the motion of material neighborhoods is purely rotational, i.e. $D \simeq 0$ in a time step, then the generalized midpoint rule may be applied to integrate the rotated stress tensor, to yield

$$
t^{n+1}-t^{n}=\Delta t \dot{t}^{n+\alpha}, \quad 0 \leqslant \alpha \leqslant 1,
$$

where $\Delta t$ is the time step. Use of the elastic relation from (27) in (29) results in

$$
\boldsymbol{t}^{n+1}-\boldsymbol{t}^{n}=\Delta t\left(E:\left(R^{\mathrm{T}} \mathrm{DR}\right)\right)^{n+\alpha} .
$$

An inspection of (30) suggests that the algorithm is unconditionally stable for values of $\alpha>\frac{1}{2}$, which is an inherent property of such a midpoint algorithm. It is also second order accurate for $\alpha=\frac{1}{2}$. Satisfactory conclusions have also been reached regarding the consistency and incremental objectivity of the algorithm in [34]. In a treatise on numerical implementation of rate constitutive relations, Hughes [35] has also proved that the term $\Delta t D^{n+\alpha}$ gives most accurate results for strain increments for $\alpha=\frac{1}{2}$ for which value only the algorithm is incrementally objective.

Equation (30) may be written in terms of displacement increments, such that the increment of the rotated stress tensor becomes

$$
t_{i j}^{n+1}-t_{i j}^{n}=E_{i j k l} R_{p k}^{n+\alpha} R_{q l}^{n+\alpha} \frac{\partial \Delta^{m} u_{p}}{\partial x_{q}^{n+\alpha}} .
$$

The updated rotated stresses should now be checked against the yield condition as shown in Table 1. For values exceeding the yield stress, the viscoplastic algorithm is applicable. Otherwise the trial elastic stress becomes the final stress state.

Viscoplastic algorithm. As seen from the discussion on product formulac, the viscoplastic algorithm should operate on (28) to relax the trial predictor stresses obtained from the elastic algorithm and also to upgrade simultaneously the loading surface through the updated values of the stresses and evolutionary internal variables. The return mapping algorithm described here is based on that proposed by Ortiz and Simo [22]. The algorithm is applicable to a wide variety of constitutive models and is based on the evaluation of response functions rather than their gradients. Such response functions are in general the stress-strain relations, the elastic modulii, the yield function and the normals to the yield surface in the stress-internal variable space. It has been seen that many of the traditional return mapping algorithms require the evaluation of the gradients of these functions which often make the calculations quite complex. The method described here is based on subcycling, i.e., breaking up the time interval into subintervals and projecting the stresses on to an updated loading surface.

In this analysis, a special assumption $\Phi(F)=F^{p}$ has been made in the overstress function for its suitability in representing metal characteristics under a wide range of experimental conditions [16]. Even though the algorithm has been constructed using a value $p=1$ for the exponent, it can be readily extended to accommodate models, for which $p>1$. Combining the last two equations of (28) and using the chain rule, the rate of change of the loading function 
Table 1

Update algorithm for viscoplasticity

At each integration point:

1. Elastic predictor:

$$
\begin{aligned}
& \left(t_{i j}\right)_{t r}^{n+1}=\left(t_{i j}\right)^{n}+E_{i j k l} R_{p k}^{n+\alpha} R_{q l}^{n+\alpha} \partial \Delta u_{p} / \partial x_{q}^{n+\alpha} \\
& (\kappa)_{l i}^{n+1}=(\kappa)^{n}
\end{aligned}
$$

2. Check for yielding: $(F)_{\mathrm{tr}}^{n+1}=(t)_{\mathrm{tr}}^{n+1}-(\kappa)_{\mathrm{tr}}^{n+1} \leqslant 0$ ?

YES: Retain stresses and go to (5)

$\left(t_{i j}\right)^{n+1}=\left(t_{i j}\right)_{\mathrm{tr}}^{n+1}, \kappa^{n+1}=\kappa^{n}$

NO: Start viscoplastic correction.

$$
i=1 \quad\left(t_{i j}\right)_{1}^{n+1}=\left(t_{i j}\right)_{\mathrm{tr}}^{n+1}, \quad \kappa_{1}^{n+1}=\kappa^{n}
$$

3. Viscoplastic corrector:

Evaluate

$$
\tau_{i}=\frac{1}{\gamma\left[E_{i j k l}\left(\partial \bar{t} / \partial t_{i j}\right)\left(\partial \bar{t} / \partial t_{k l}\right)+E_{p}\right]_{i}}
$$

Find $\Delta t_{i}$ for bounded $\Delta t_{i} / \tau_{i}$

Update

$$
\begin{aligned}
\left(t_{i j}\right)_{i+1}^{n+1} & =\left(t_{i j}\right)_{i}^{n+1}-\gamma \tau_{i} F_{i}^{n+1} E_{i j k l}\left(1-\mathrm{e}^{-\Delta t_{i} / \tau_{i}}\right) \partial \bar{t} / \partial t_{k l} \\
(\kappa)_{i+1}^{n+1} & =(\kappa)_{i}^{n+1}-\gamma \tau_{i} F_{i}^{n+1} E_{p}\left(1-\mathrm{e}^{-\Delta t_{i} / \tau_{i}}\right) \\
t_{i+1} & =t_{1}+\Delta t_{i}
\end{aligned}
$$

4. Process termination:

$$
\begin{array}{ll}
t_{i+1}<\Delta t & \text { go to }(3) i \rightarrow i+1 \\
t_{i+1}=\Delta t & \text { go to (5) }
\end{array}
$$

5. Rotate the stress by $\boldsymbol{R}^{n+1}$ to obtain $\sigma^{n+1}$ $\sigma^{n+1}=\left(R^{n+1}\right) t^{n+1}\left(R^{t}\right)^{n+1}$

$\left(F=\bar{t}-\kappa\left(W_{\mathrm{p}}\right)\right)$ may be written as

$$
\frac{\partial F}{\partial t}=-\gamma F\left[E_{i j k l} \frac{\partial \bar{t}}{\partial t_{i j}} \frac{\partial \bar{t}}{\partial t_{k l}}+E_{p}\right] \text { or } \frac{\partial F}{\partial t}=-\frac{F}{\tau},
$$

where $\tau\left(=1 /\left(\gamma\left[E_{i j k l} \partial \bar{t} / \partial t_{i j} \partial \bar{t} / \partial t_{k l}+E_{p}\right]\right)\right)$ is an instantaneous relaxation time. For values of $p>1$, the corresponding relaxation time is obtained by dividing the above expression by the exponent $p$. The relaxation time depends on the derivative of equivalent stress with respect to the stress components and may in general vary over a time step. So a closed form integration for (32) is not always possible. Therefore we subdivide the time interval into a number of segments. Assuming $\tau$ to remain constant in each segment, (32) may be integrated to obtain the yield function at the end of the $i$-th segment as

$$
F_{i+1}^{n+1}=F_{i}^{n+1} \mathrm{e}^{-\Delta t_{i} / \tau_{i}},
$$

where $\tau_{i}$ refers to the relaxation time at the beginning of the segment. The rotated Cauchy stress and the work hardening parameter may then be updated to the end of the $i$-th segment by integrating the straight segments of the return path, the second and last equations of the set (28) to give

$$
\begin{aligned}
& \left(t_{i j}\right)_{i+1}^{n+1}=\left(t_{i j}\right)_{i}^{n+1}-\Delta \delta_{i} E_{i j k l}\left(1-\mathrm{e}^{-\Delta t_{i} / \tau_{i}}\right) \partial \bar{t} / \partial t_{k l}, \\
& (\kappa)_{i+1}^{n+1}=(\kappa)_{i}^{n+1}+\Delta \delta_{i} E_{p}\left(1-\mathrm{e}^{-\Delta t_{i} / \tau_{i}}\right),
\end{aligned}
$$


where $\Delta \delta_{i}=\gamma \tau_{i} F_{i}^{n+1}$. Equations (34) defining the return path are dissipative in nature and the corresponding trajectories are contractive since the yield function is assumed to be convex and the plastic flow is associative. The resulting algorithm is therefore unconditionally stable under these conditions.

In updating the stresses in this manner, one has to be cautious above the ratio $\Delta t_{i} / \tau_{i}$ because of its use in the exponential form. In the term $\Delta t_{i} / \tau_{i}$ is reasonably big $(\sim 10)$, the term $\left(1-\mathrm{e}^{-\Delta t_{i} / \tau_{i}}\right)$ tends to 1 and this results in a collapse in the algorithm. On the other hand, a very small value of $\Delta t_{i}$ making $\Delta t_{i} / \tau_{i}$ small, may result in a lot of segments within each time step and this becomes computationally expensive. Thus an optimum value of $\Delta t_{i} / \tau_{i}$ has to be established in every segment before performing the updating. The above algorithm is relatively straightforward and eliminates the need for iteration. If carefully implemented, this proves to be a very efficient way to update stress and internal variables. Table 1 shows a summarized form of the update algorithm for implementation in the finite element analysis. However, one shortcoming of this update algorithm is that in the process of meeting the time step requirement $\left(\Sigma \Delta t_{i}=\Delta t\right)$, the stress points may not exactly reach the loading surface. It should be noted here, that this updating is carried out for material particles at integration points of an element.

\section{Approximate linearization of the boundary value problem of momentum balance}

In the preceding sections we have discussed the various ingredients required for the finite element modeling of large deformation analysis of materials exhibiting inelastic response. It can be easily seen from the equations presented, that the combined set of equations is highly nonlinear in their kinematic and constitutive behavior. It is therefore imperative to linearize the set of equations before undergoing the solution process with iterative methods. To do this, we start with the weak form of the momentum balance equation in the midstep configuration (15) and express it as a scalar valued function $G$, where

$$
\begin{aligned}
G\left(\Delta^{\mathrm{m}} u, \bar{u}\right)= & \int_{\Omega\left(t_{n+a}\right)} \sigma_{i i} \frac{\partial \bar{u}_{i}}{\partial x_{j}} \mathrm{~d} \Omega+\int_{\Omega\left(t_{n+a}\right)} \rho\left(V_{j}-W_{j}\right) \frac{\partial V_{i}}{\partial x_{j}} \bar{u}_{i} \mathrm{~d} \Omega-\int_{\Omega\left(t_{n+a}\right)} \rho G_{i} \bar{u}_{i} \mathrm{~d} \Omega \\
& -\int_{\Gamma\left(t_{n+a}\right)} T_{i} \bar{u}_{i} \mathrm{~d} \Gamma=0 .
\end{aligned}
$$

All the tensors occurring in the equilibrium equation have a functional dependence on the motion. Therefore in general, the above scalar form is nonlinear with respect to the displacement of a material particle $\Delta^{\mathrm{m}} u\left(=\left(x^{*}\right)^{n+1}-\left(x^{*}\right)^{n}\right)$, see Fig. 1. Here $x^{*}$ corresponds to the path of a material point coinciding with the grid point at $t_{n+\alpha}$. A consistent linearization with respect to the reference material point displacement $\Delta^{\mathrm{m}} \dot{u}$ may be expressed based on Taylor's formula for $C^{1}$ functions as

$$
L[G]_{\Delta^{m_{\dot{u}}}}=\boldsymbol{G}\left(\Delta^{\mathrm{m}} \dot{u}, \bar{u}\right)+\mathrm{D} \boldsymbol{G}\left(\Delta^{\mathrm{m}} \dot{u}, \bar{u}\right) \cdot u=0,
$$

where $\mathrm{D} G \cdot u$ refers to the directional derivative of $G\left(\Delta^{\mathrm{m}} \dot{u}, \bar{u}\right)$ in the direction of incremental 
displacement $\boldsymbol{u}$ and may be written as

$$
D G \cdot u=\frac{\partial}{\partial \varepsilon}\left[G\left(\Delta^{\mathrm{m}} \dot{u}+\varepsilon u\right)\right]_{\varepsilon=0},
$$

with $\varepsilon$ being a scalar parameter. Assuming that the domain $\Omega\left(t_{n+\alpha}\right)$ and the grid point location $x^{n+\alpha}$ in the ALE description are independent of the particle displacement $\Delta^{\mathrm{m}} u$, the directional derivative of the function $\boldsymbol{G}$ given by (35) is expressed as

$$
\begin{aligned}
\mathrm{D} G \cdot u= & \left.\int_{\Omega\left(t_{n+\alpha}\right)} \operatorname{tr}[(\mathrm{D} \sigma \cdot u) \cdot \nabla \bar{u}] \mathrm{d} \Omega+\int_{\Omega\left(t_{n+\alpha}\right)}[\mathrm{D}(\rho(V-W) \cdot \nabla) V) \cdot u\right] \cdot \bar{u} \mathrm{~d} \Omega \\
& -\int_{\Omega\left(t_{n+\alpha}\right)}[\mathrm{D}(\rho G) \cdot u] \bar{u} \mathrm{~d} \Omega-\int_{\Gamma\left(t_{n+\alpha}\right)}(\mathrm{DT} \cdot u) \cdot \bar{u} \mathrm{~d} \Gamma=-G\left(\Delta^{\mathrm{m}} \dot{u}, \bar{u}\right) .
\end{aligned}
$$

A rigorous linearization performed with respect to the motion $\Delta^{\mathrm{m}} u$ results in a lot of terms in the construction of the tangent stiffness matrix. However, as will be discussed in the following section, the iteration method used requires only a first approximation to the stiffness matrix. Therefore we have chosen to make certain relevant assumptions in the linearization process. First, because of the viscoplastic incompressibility condition, we may assume that the density does not change considerably, and therefore may be treated as a constant. In order to find the directional derivative of the Cauchy stress tensor, a functional dependence to the incremental displacement $\Delta^{\mathrm{m}} u$ is introduced through the rotated stress-strain relation (11). Since an explicit dependence of $\boldsymbol{R}\left(=\boldsymbol{F} \boldsymbol{U}^{-1}\right)$ on the incremental displacement is cumbersome in nature, an assumption that $R^{n+\alpha}$ remains unchanged in the vicinity of $\Delta^{\mathrm{m}} \dot{u}$ is made. In order to linearize the stress tensor, we recall that the Cauchy stress tensor in an intermediate configuration may be expressed in terms of the rotated stress tensor by the relation

$$
\sigma^{n+\alpha}=\left(R^{\mathrm{T}}\right)^{n+a}\left(t^{n}+\alpha \Delta^{\mathrm{m} t} t\right) R^{n+a},
$$

where $\Delta^{\mathrm{m}} t$ is the increment of the rotated stress at a material point which coincides with a grid point at $\boldsymbol{x}^{n+t}$ in the mid-step configuration. This incremental stress may be obtained by an integration of the material constitutive relations (11) using the techniques discussed in Section 5 to yield the approximate relation

$$
\Delta^{m t_{i j}}=R_{p k}^{n+\alpha} R_{q l}^{n+\alpha} E_{i j k l} \frac{\partial \Delta^{\mathrm{m}} u_{p}}{\partial x_{q}^{n+\alpha}}-\left(\gamma F E_{i j k l} \frac{\partial \bar{t}}{\partial t_{k l}}\right)^{n+\alpha} \Delta t .
$$

A consistent linearization of the increment of rotated stress tensor may then be carried out using the standard techniques to yield

$$
\mathrm{D}\left(\Delta^{\mathrm{m} t} t\right) \cdot \boldsymbol{u}=\hat{\boldsymbol{E}}: \Delta \hat{\varepsilon}
$$

where $\Delta \hat{\varepsilon}_{i j}=\frac{1}{2} R_{p i} R_{q j}\left[\partial u_{p} / \partial x_{q}+\partial u_{q} / \partial x_{p}\right]$. Here $\hat{\boldsymbol{F}}$ is the tangent modulus obtained as an inverse of a fourth order tensor $\hat{C}$ expressed by the relation 


$$
\hat{C}_{i j k l}=C_{i j k l}+\gamma \Delta t\left[\frac{1}{\left(1+\gamma E_{p} \Delta t\right)} \frac{\partial \bar{t}}{\partial t_{i j}} \frac{\partial \bar{t}}{\partial t_{k l}}+(\bar{t}-\kappa) \frac{\partial^{2} \bar{t}}{\partial t_{i j} \partial t_{k l}}\right]
$$

with $C_{i j k l}$ representing the elastic compliance.

In a similar fashion, it is possible to linearize the traction term in the contact boundary from (23) as

$$
\mathrm{DT} \cdot u=-\alpha k_{n}\left(u \cdot n^{n+\alpha}\right) n^{n+\alpha} .
$$

Substituting the linearized forms of the above components into (38), the approximate linearized form of the equilibrium equation may then be written as

$$
\begin{aligned}
& \int_{\Omega\left(t_{n+\alpha}\right)} \alpha R_{j k} R_{i l} R_{p m} R_{p n} \hat{E}_{k l m n} \frac{\partial u_{p}}{\partial x_{q}} \frac{\partial \bar{u}_{i}}{\partial x_{j}} \mathrm{~d} \Omega+\int_{\Omega\left(t_{n+\alpha}\right)} \frac{\partial t_{j i}}{\partial u_{k}} u_{k} \frac{\partial \bar{u}_{i}}{\partial x_{j}} \mathrm{~d} \Omega \\
& \quad+\frac{1}{\Delta t} \int_{\Omega\left(t_{n+\alpha}\right)} \rho u_{j} \frac{\partial V_{i}}{\partial x_{j}} \bar{u}_{i} \mathrm{~d} \Omega+\frac{1}{\Delta t} \int_{\Omega\left(t_{n+\alpha}\right)} \rho\left(V_{j}-W_{j}\right) \frac{\partial u_{i}}{\partial x_{j}} \bar{u}_{i} \mathrm{~d} \Omega \\
& \quad+\int_{\Gamma_{\mathrm{C}\left(t_{n+\alpha}\right)} \alpha k_{n} u_{j} n_{j}^{n+\alpha} n_{i}^{n+\alpha} \bar{u}_{i} \mathrm{~d} \Gamma}=\int_{\Omega\left(t_{n+\alpha}\right)} \rho G_{i} \bar{u}_{i} \mathrm{~d} \Omega+\int_{\Gamma\left(t_{n+\alpha}\right)} T_{i} \bar{u}_{i} \mathrm{~d} \Gamma-\int_{\Omega\left(t_{n+\alpha}\right)} \sigma_{j i} \frac{\partial \bar{u}_{i}}{\partial x_{j}} \mathrm{~d} \Omega \\
& \quad-\int_{\Omega\left(t_{n+\alpha}\right)} \rho\left(V_{j}-W_{j}\right) \frac{\partial V_{i}}{\partial x_{j}} \bar{u}_{i} \mathrm{~d} \Omega .
\end{aligned}
$$

It is this equation (44), that will be used in the iteration procedures for obtaining the solution to the boundary value problem.

\section{Numerical implementation}

A wide variety of numerical details have to be considered in the construction of a global solution strategy for solving large deformation problems using the model that we have discussed so far. In this section we focus on a few salient features of the solution process.

\subsection{Iteration scheme}

The physical grid point domain is discretized into isoparametric eight node brick elements with variables interpolated by the Lagrangian shape functions. It is to be remembered that the elements are essentially made up of grid points rather than of material points. Hence the variables have to be appropriately updated before interpolation. The method utilized in doing so has been discussed later on in this section.

It is obvious that the linearized form of the principle of virtual work (44) has to be solved for the displacement increment $\Delta^{\mathrm{m}} u$ iteratively. A judicious choice of the iteration scheme is necessary from an efficiency and accuracy point of view. Several studies on iterative 
techniques $[36,37]$ suggest that the use of a full Newton-Raphson iteration is not very effective in general geometric and material nonlinear response calculations. It requires accurate evaluation and updating of the effective stiffness matrix in every iteration step, which in the present case may become computationally very expensive due to the several contributing terms. In general, for a system of $n$ nonlinear equations, it requires a large number of computations $\left(\mathrm{O}\left(n^{3}\right)\right)$ per iteration step. Thus, from an efficiency point of view, this calls for relatively large load steps which again are restricted by stability and accuracy considerations. A remedy to the drawbacks of the full Newton scheme is offered in the modified Newton's method. In this method the stiffness matrix is not updated in every time step but only from time to time. However, problems of slow convergence and divergence are frequently encountered with these methods. As an alternative to the different forms of Newton iteration, a class of methods known as matrix update methods or quasi-Newton methods have been found suitable for iterative solution of a system of nonlinear equations. For a system of $n$ nonlinear equations, these methods require only $n$ scalar functional evaluations and reduces the number of arithmetic calculations to $\mathrm{O}\left(n^{2}\right)$. In these methods, the Jacobian matrix in the Newton iteration is replaced by an approximation matrix. The above iteration methods have been found to be most effective in this analysis. We now elaborate on the steps involved, in the solution of the boundary value problem of interest.

Applying the finite element approximations in the discretized domain, the linearized equation (44) may be written for the $i$-th iteration step as

$$
{ }^{1+\alpha}{ }^{\prime}[K]^{i-1}\{\Delta U\}={ }^{i+\alpha \Delta t} R-{ }^{i+\alpha \Delta t} F^{i-1},
$$

where $\Delta U=\Delta^{m} U^{i}-\Delta^{m} U^{i-1}$ refers to the correction in the incremental material point displacement corresponding to a grid point in the intermediate configuration. The quasi-Newton iteration then proceeds in the following steps.

STEP 1. With an initial guess for $\Delta^{m} U$, evaluate the right-hand side ${ }^{1+\alpha \Delta t} R-{ }^{1+\alpha} \Delta t F^{0}$ of (45) and also the initial stiffness matrix ${ }^{1+\alpha} \Delta^{\Delta_{t}}[K]^{0}$ using (18) to establish mid-step variables. The initial guess to the displacement increment is obtained by solving the incremental form of the weak form using explicit time integration.

STEP 2. Evaluate a displacement vector $\boldsymbol{U}$ which defines a direction for the actual displacement increment vector as

$$
U={ }^{1+\alpha \Delta}\left[K^{-1}\right]^{i-1}\left({ }^{1+\alpha \Delta} R-{ }^{1+\alpha \Delta t} F^{i-1}\right) .
$$

STEP 3. Perform a line search in the direction of $\boldsymbol{U}$ to satisfy equilibrium. In this step a scalar parameter $s(0 \leqslant s \leqslant 1)$, defining the displacement increment as

$$
\Delta^{\mathrm{m}} U^{i}=\Delta^{\mathrm{m}} \boldsymbol{U}^{i-1}+s \boldsymbol{U},
$$

is varied to make the projection of the off balance loads in the direction of $\boldsymbol{U}$ approximately zero.

STEP 4. Calculate the values of the off balance loads using the new values of $\Delta^{\mathrm{m}} U$. Also, the inverse of the stiffness matrix is updated using the BFGS or Broyden's update formulae 
$[37,38]$. It has been observed in our analysis that the Broyden's update performs more effectively and accurately for the nonsymmetric matrix updates required in this analysis.

STEP 5. A convergence check is done at the end of each iteration for displacements, out of balance load vectors and incremental internal energy. Divergence results when out of balance loads exceed the initial value and is remedied by immediate stiffness reformation. The displacement norm and internal energy are checked against their initial values within some preset tolerances (in our case and $\varepsilon_{\mathrm{D}}=10^{-3}$ and $\varepsilon_{\mathrm{E}}=10^{-2}$ ). More details on the convergence criteria may be looked up in [39].

\subsection{Updating scheme}

A very important consideration to be given in the ALE description is the updating of variables to the nodal points of the arbitrary mesh in the intermediate and final configurations. In this analysis, there exist two types of variables: (a) variables which have explicit dependence on the mesh movement (e.g. the grid velocity $W$ ) and (b) variables which are material path dependent and their dependence on the mesh movement is not explicit. For this second class of variables, nodal point values have to be obtained through interpolation.

The variables of the first kind are easily updated to the mid-step configuration and hence to the end configuration based on information on nodal points alone, e.g.

$$
W^{n+\alpha} \simeq \Delta^{8} x / \Delta t
$$

where $\Delta^{\mathbf{g}} \boldsymbol{x}$ is the grid displacement in a time step. The values at the grid points in the $(n+1)$-th configuration are then calculated by extrapolation using (18). However for variables of the second kind, a much more involved updating process is necessary. This is outlined below.

(a) First, pseudo-material elements are constructed at time $t_{n+\alpha}$ by projecting the material displacements $\left(\alpha \Delta^{\mathrm{m}} u\right)$ from the nodal points at time $t_{n}$ to the intermediate configuration, as in a pure Lagrangian case.

(b) Next, the material element in which a grid (nodal) point at location $x^{n+\alpha}$ belongs is found. This is done by checking iteratively for the values of the normalized local coordinates of the grid point in the mapped master element corresponding to a material element. If the magnitudes of all of the normalized coordinates are less than or equal to 1 , then the material element in which a grid point belongs may be deciphered. The values of the variables, such as $\Delta^{\mathrm{m}} u^{*},\left(\rho^{*}\right)^{n},\left(\sigma^{*}\right)^{n}$, etc., in the $n$-th configuration, for a material point which coincides with the grid point in the $(n+\alpha)$-th configuration are then obtained by interpolation. Figure 2 gives an idea of the variables in different configurations.

(c) Corresponding to the displacements $\Delta^{\mathrm{m}} u^{*}$ as shown in the figure, the stress and velocity increments are evaluated for a material point coinciding with the grid point at time $t_{n+\alpha}$. The intermediate values are calculated from these increments using (39) and the relation $V^{n+\alpha}=\Delta^{m} u^{*} / \Delta t$ (see also [17]). Iterative correction for $\Delta^{m} u^{*}$ is continued till convergence is achieved.

(d) Finally the material variables are updated to the grid configuration at $t_{n+1}$ using the ALE relations given by (20). Figure 4 gives a detailed flow chart of the entire solution process. An important observation with regards to the updating of material point variables, 

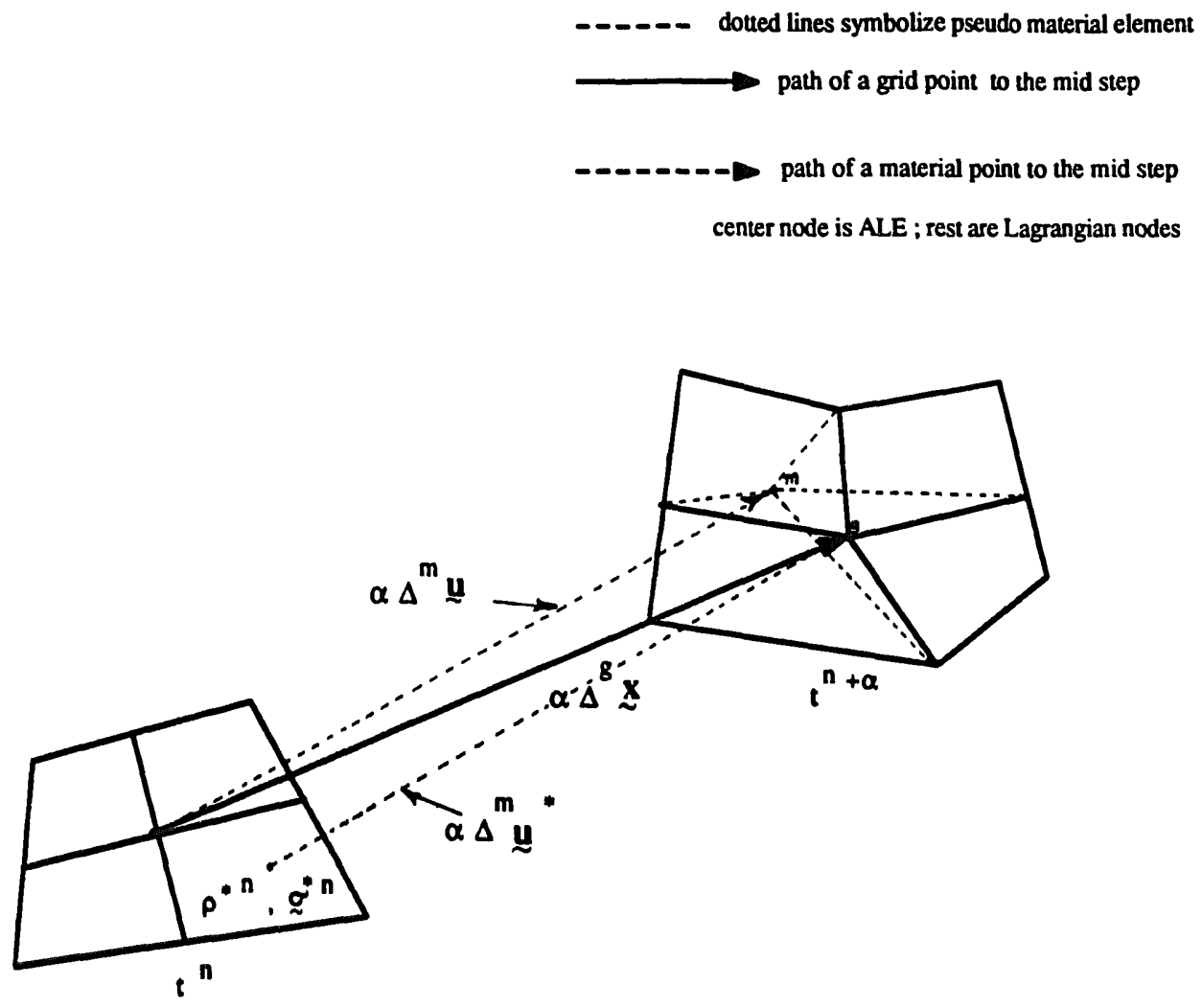

Fig. 2. Updating variables to the ALE node at mid-step.

especially Cauchy stress and rotated Cauchy stress tensors, is worth mentioning. The variables mentioned above are initially calculated at the integration points of an element in the FEM model from displacement values at the nodal points. If the values of these variables are then projected to the nodal points for carrying material information to the subsequent steps by methods like least squares or weighted averaging, severe instabilities are noticed after sufficient number of steps. A simple way to avoid such instabilities is by storing stress values at the integration points of an element and updating directly to the corresponding points at subsequent steps.

In moving the grid points at boundary, the condition that the grid boundary encompasses the material at all times (17) must be enforced. For a smooth surface the normal is first evaluated at the nodal point in an average sense from the surrounding elements in $\Omega\left(t_{n+\alpha}\right)$ (see Fig. 3). The normal component of the displacement is evaluated as $\Delta^{\mathrm{m}} u_{n}=\Delta^{\mathrm{m}} u \cdot n$. For a prescribed value of the tangential component of grid displacement $\Delta^{8} x_{1}$, the total grid displacement at a nodal point is specified as

$$
\Delta^{g} x=\Delta^{m} u_{n} n+\Delta^{g} x
$$

For sliding boundaries with corners, special attention must be given to the fact that more than one normal might exist and hence (49) must be specified accordingly. 


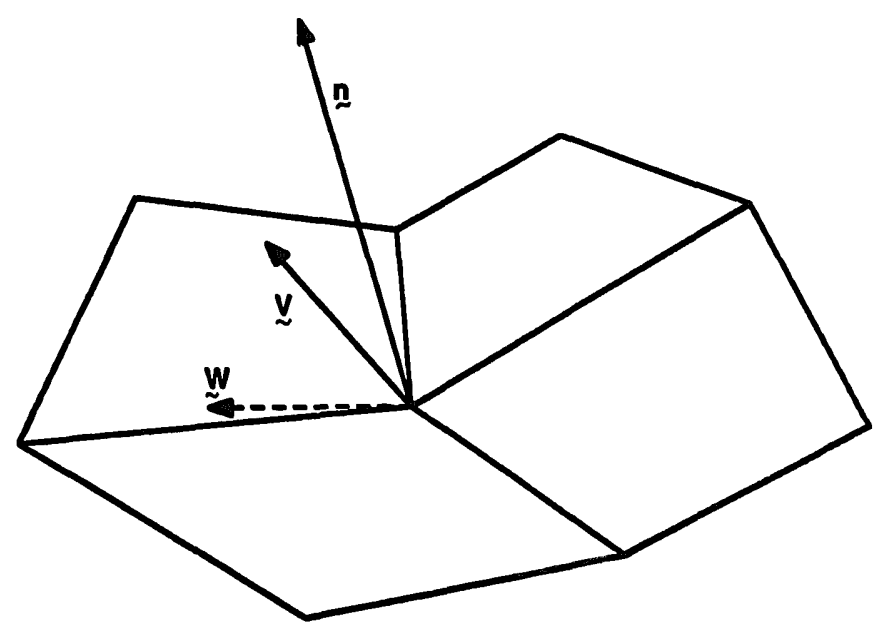

Fig. 3. Representation of a normal and velocities for applying the boundary constraint condition.

\subsection{Reduced integration procedures}

It has been a common experience with nearly incompressible materials that full integration of the stiffness matrix using the Gaussian quadrature rules for certain constraint problems can give rise to physically meaningless locked solutions. Methods of selective reduced integration are applied to alleviate this shortcoming. However for general anisotropic material the segregation of the stiffness matrix into volumetric and other terms is not always feasible. Following the procedure suggested by Hughes [40] the strain-displacement matrix $B$ is modified to be formed by a combination of terms formed by reduced and full integration. The stress increments are computed by separating the dilatation part of the strain rate from the deviatoric part and evaluating the incremental arising from the former by one point reduced integration.

Another phenomenon that is noticed in bending dominated problems with full integration, is the so called 'shear locking' due to an excessive contribution of shear to the internal energy. Selective reduced integration with hourglass control has been used to control this locking phenomenon induced by shear [41]. However, the selective reduced integration may not provide desirable approximations for elements which are at an angular orientation with respect to the global axes or for problems which predominantly involve torsion. A remedy has been suggested in a recent paper by Koh and Kikuchi [42] in the form of directional reduced integration (DRI). In this method, the underintegration criterion of strains is determined from the geometry of an element, rather than from the physical component itself. Such a technique has been adopted in this analysis because of its suitability in large deformation problems involving rotation. Details of this technique may be obtained in [42]. 


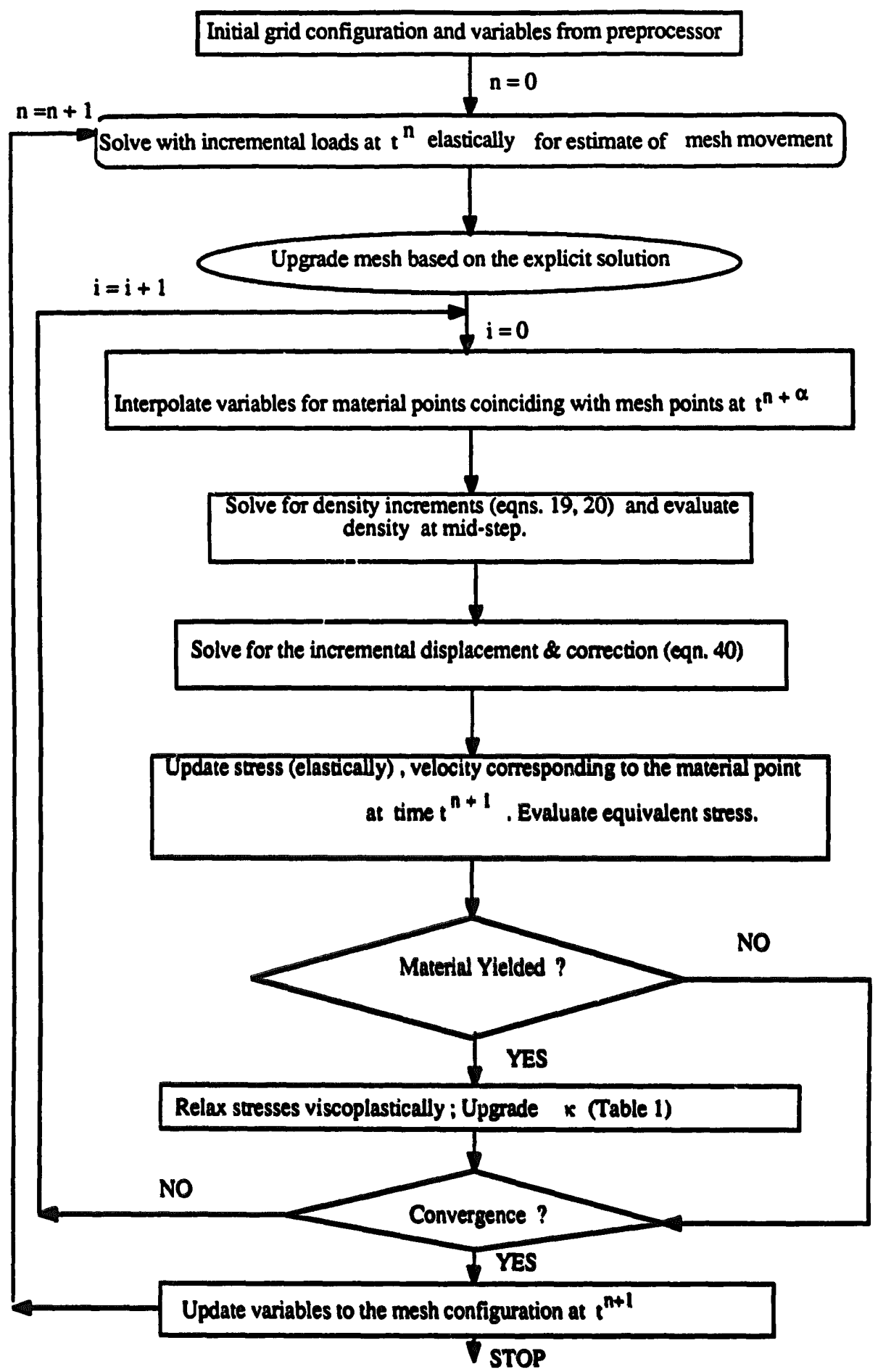

Fig. 4. Flow chart representing the analysis.

\section{Numerical examples}

A Fortran computer code has been developed for the implementation of the aforementioned algorithm. Using this code, a number of different problems were solved to verify the 
validity of the algorithms as well as the code. All the examples were executed on Apollo DN 3000 and DN 4000 computers. In the presentation of the examples we take a sequential approach to the development of different aspects of the entire algorithm. That is, we first test the elastic-viscoplastic algorithms for large deformation by solving a few standard benchmark type problems and then we explore the flexibility of the ALE description for elasticviscoplastic material behavior by numerically experimenting with several illustrative examples with particular emphasis to metal forming processes. This section is therefore divided into two subsections namely (a) elastic-viscoplastic problems and (b) metal forming type problems using the ALE description. Though the formulation is of general nature, all the examples mentioned in this section have been carried out in the midstep configuration using the value of $\alpha=\frac{1}{2}$. The three-dimensional elements used to discretize the domains in all the examples are the eight-node Lagrangian elements.

\subsection{Elastic-viscoplastic problems}

EXAMPLE 1. Tension test: Stress and strain controlled tests are performed for a bar with material parameters as follows: $E=10,000 \mathrm{psi}, \nu=0.3, \sigma_{\mathrm{Y}}=10 \mathrm{psi}, E_{t}$ (elastic-plastic modulus) $=3333.3333 \mathrm{psi}, \gamma=0.001 \mathrm{sec}^{-1}$.

In the stress controlled tension test, a load of $15 \mathrm{lbs}$ is applied at one end of the bar over a time period of one second divided into twenty equal segments. Figure 5 shows the creep response of the solid for various values of $\gamma$. The results obtained are compared with those in [43] and a good agreement is cbtained. The variation in static yield or work hardening parameter $\kappa$ is shown in Fig. 6.

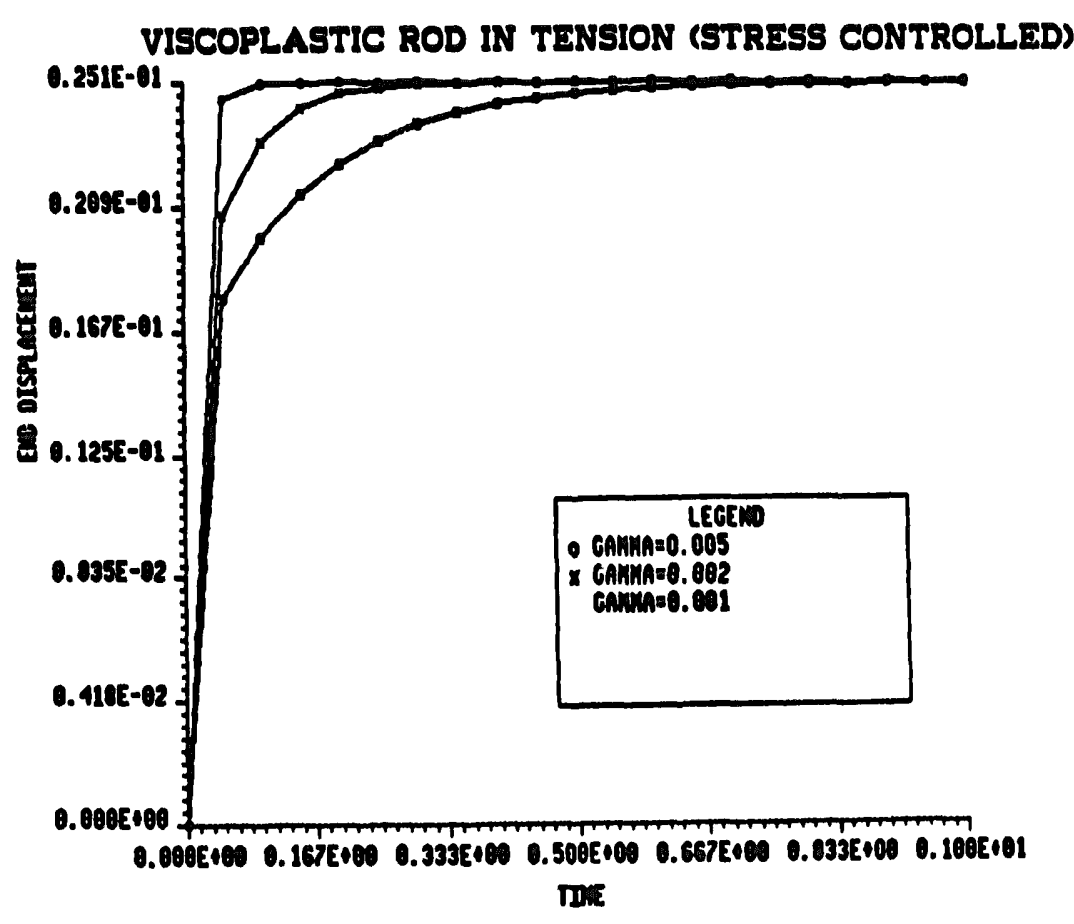

Fig. 5. Extension (in) versus time (sec) for varying viscosity parameter. 


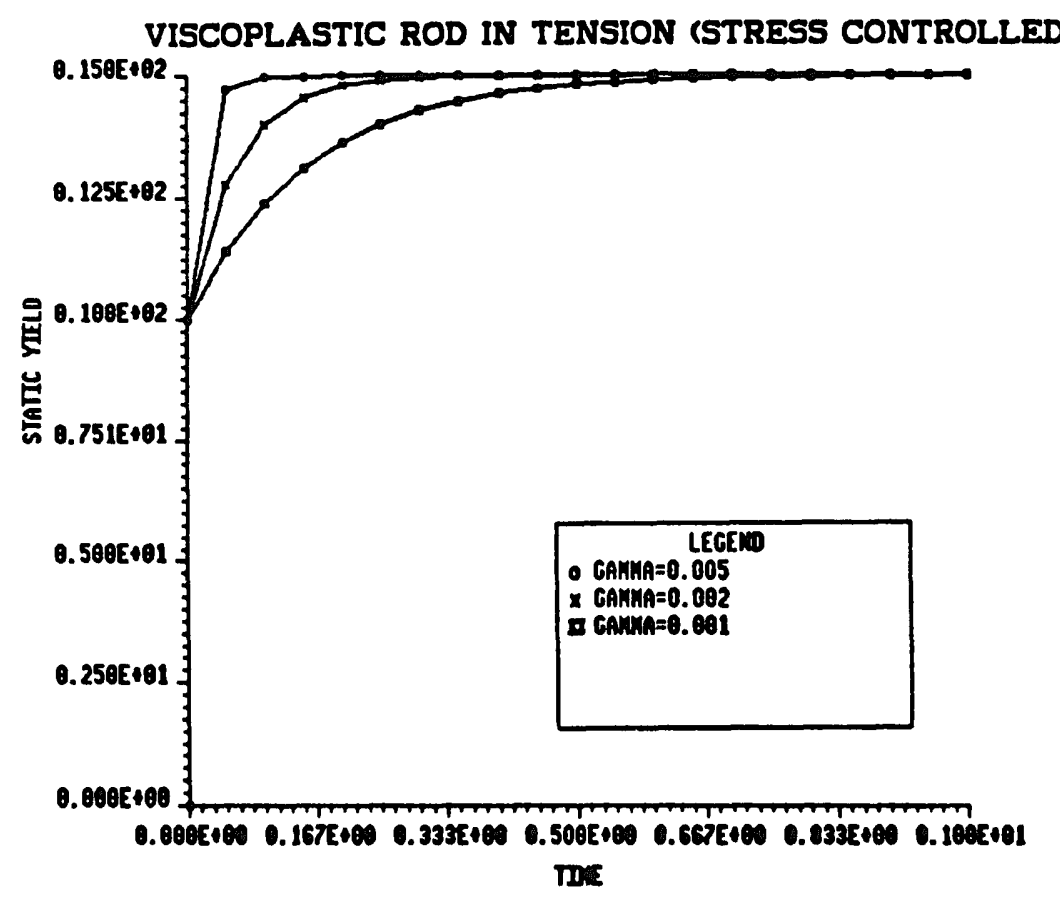

Fig. 6. Static yield (psi) versus time (sec) for varying viscosity parameter.

Next a strain controlled tension test is analyzed. In this example, one end of the bar is assumed to be pulled with a constant velocity (strain rate) $0.005 \mathrm{in} / \mathrm{sec}$ for half the total time after which the end is held fixed. The stress relaxation characteristics are clearly seen from Fig. 8 and the response of yield stress from Fig. 7. Finally the effect of strain rates on the stress and work-hardening characteristics are shown in Figs. 9 and 10 and these seem to be in good agreement with the experimentally observed results.

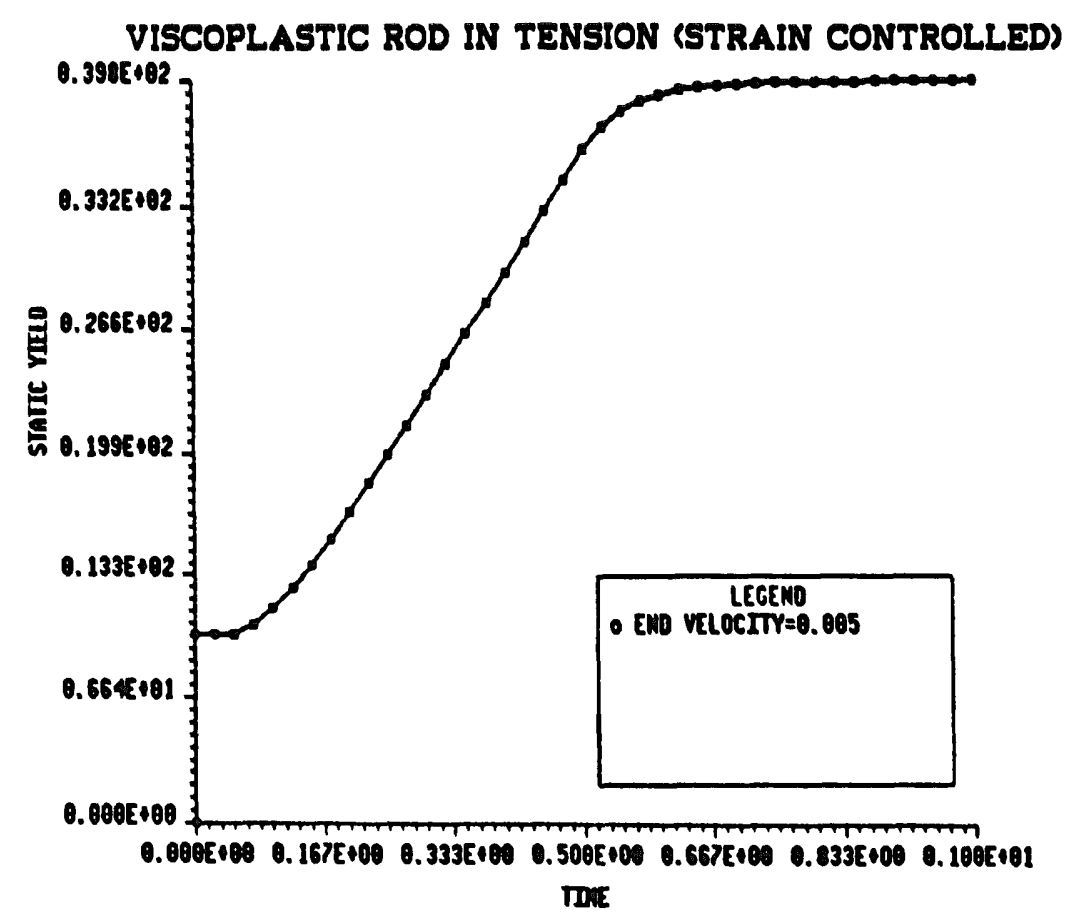

Fig. 7. Static yield (psi) versus time (sec) in tension test with relaxation. 


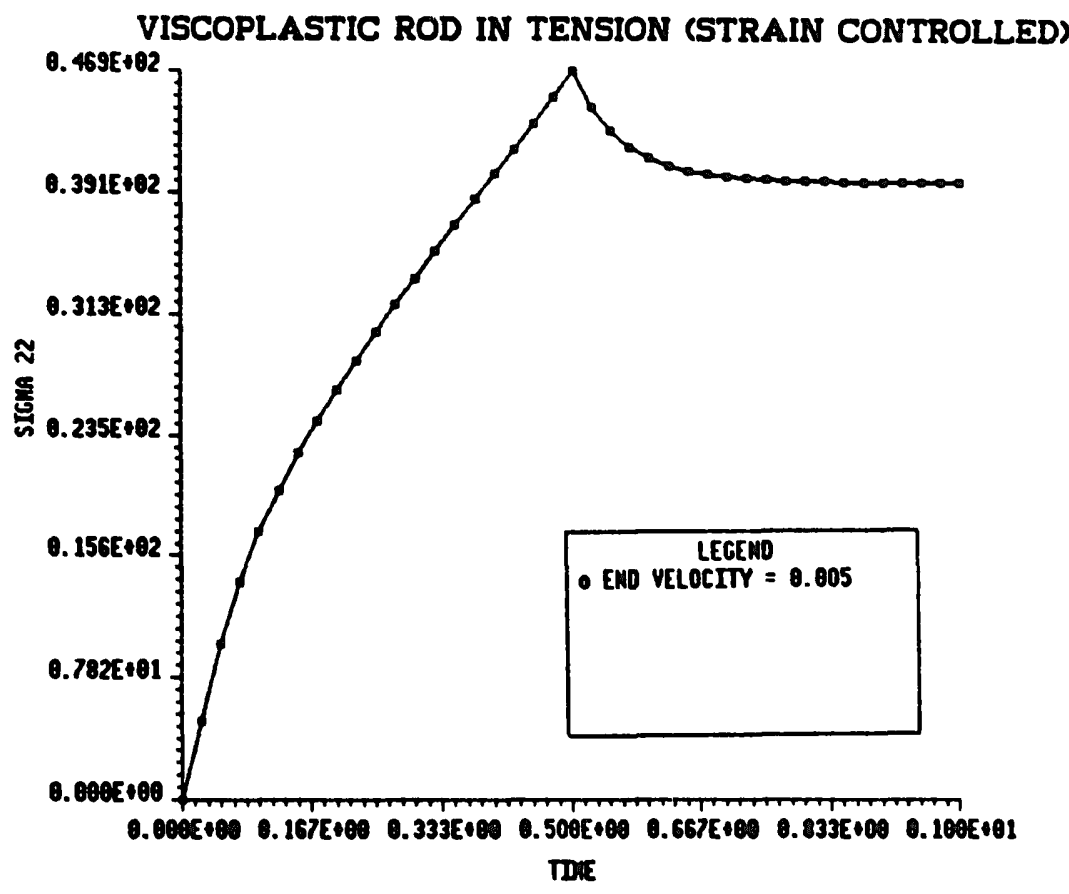

Fig. 8. Axial stress (psi) versus time (sec) in tension test with relaxation.

EXAMPLE 2. Simple shear test. A simple shear test on an elastic-viscoplastic cube, as shown in Fig. 12 is executed. The major objective of this numerical experiment is to observe whether any oscillation in the shearing stress occur with the model, as is observed in the traditional Jaumann's rate description. The top surface of the cube is moved by four times the height of

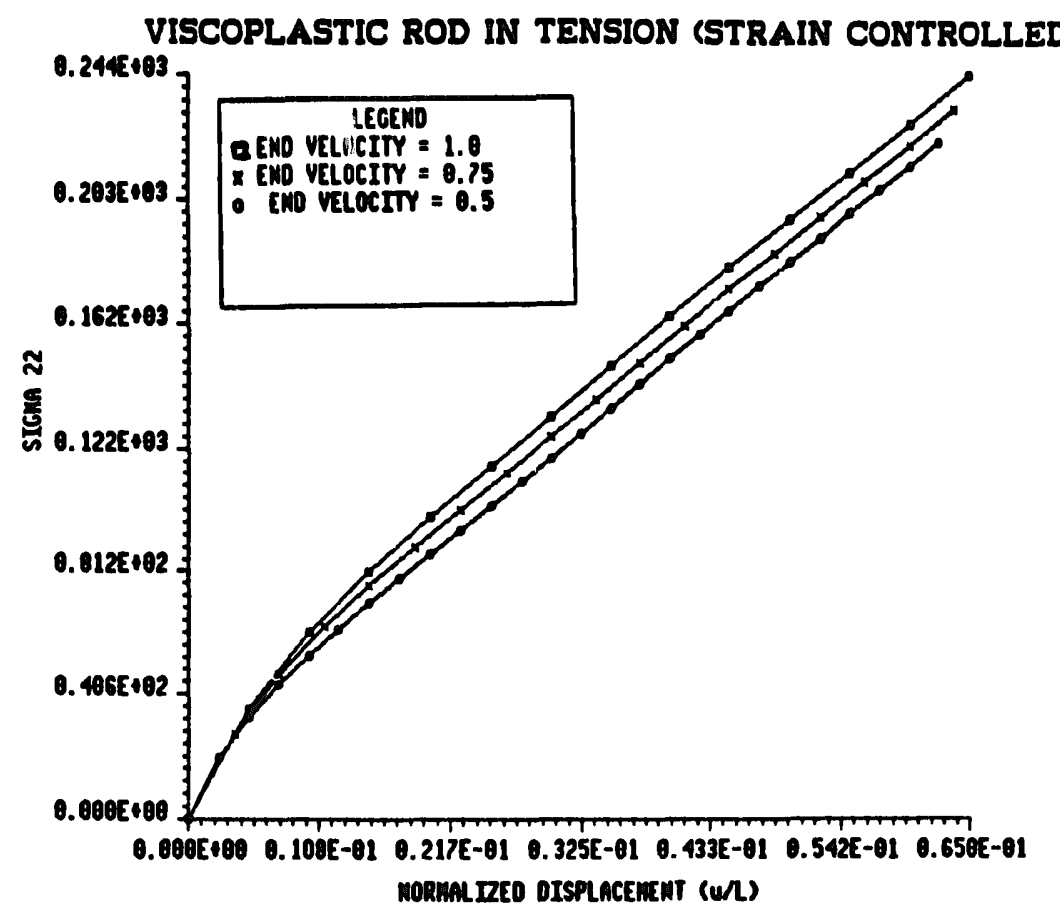

Fig. 9. Axial stress (psi) versus normalized displacement for varying strain rates. 


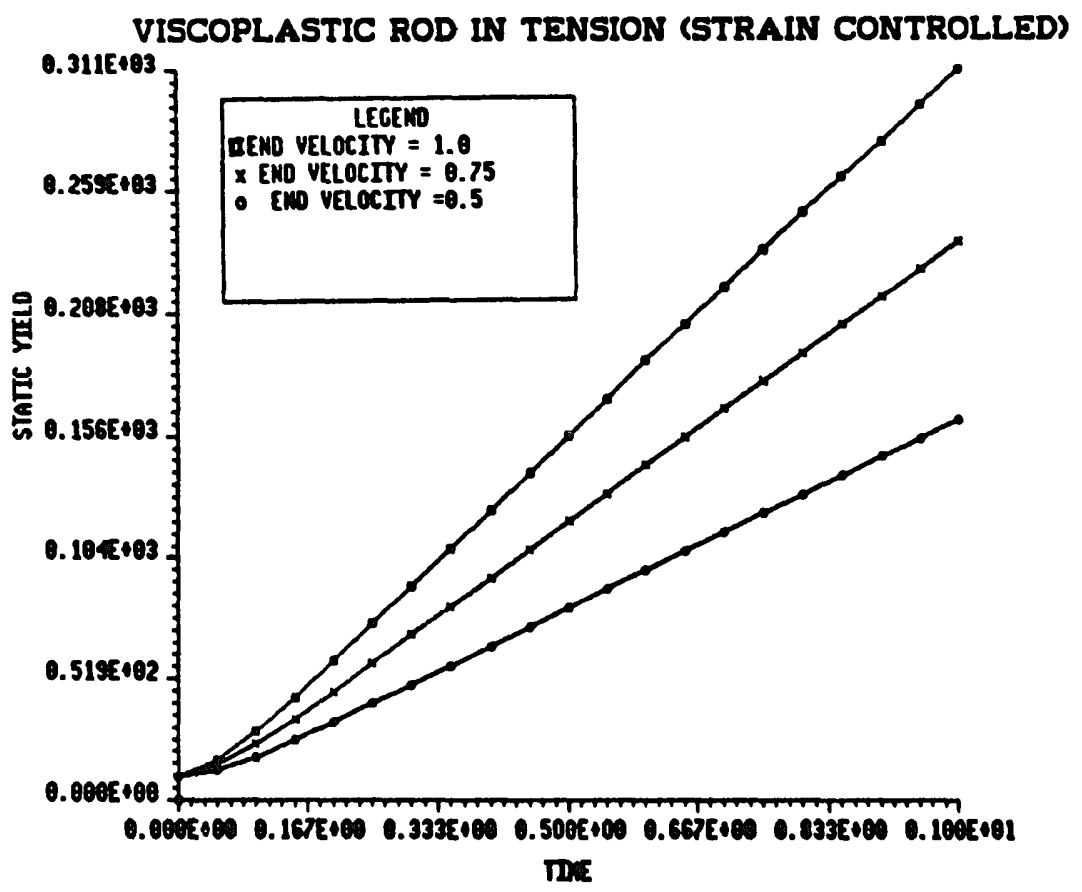

Fig. 10. Static yield (psi) versus time (sec) for varying strain rates.

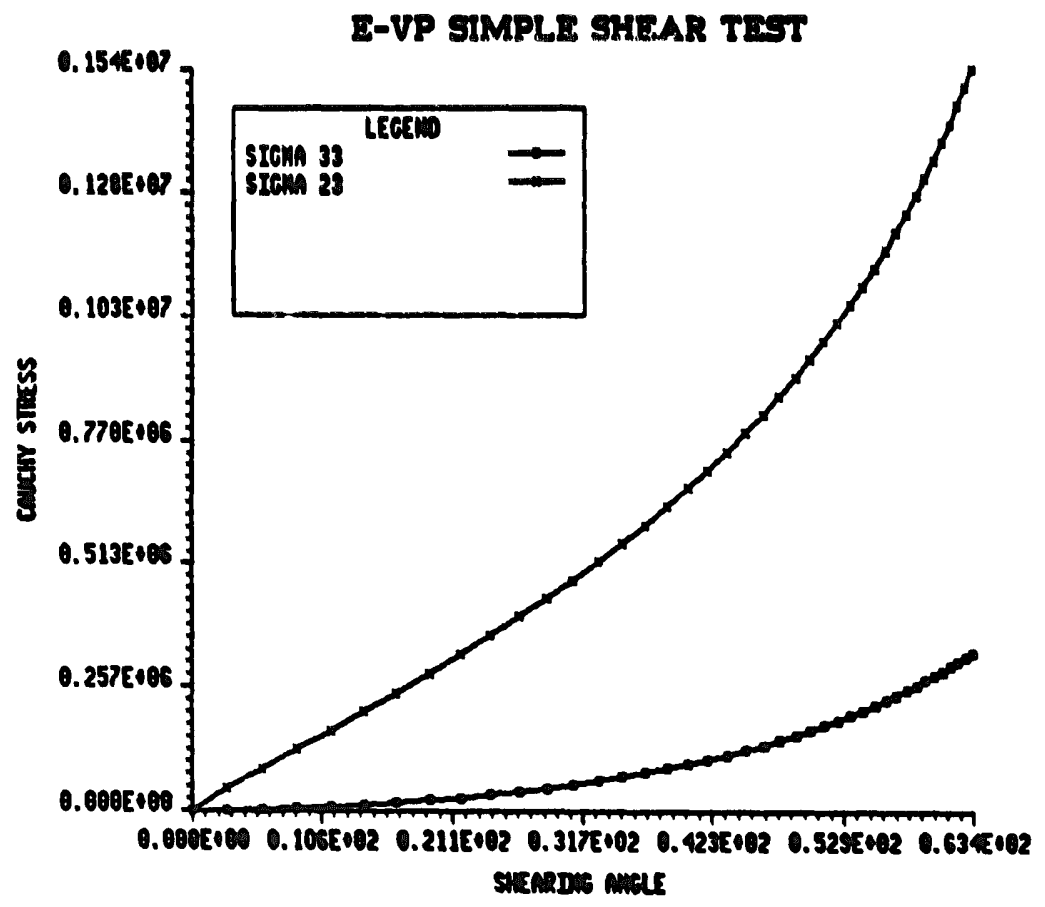

Fig. 11. Stresses (psi) versus shear strain in simple shear test. 

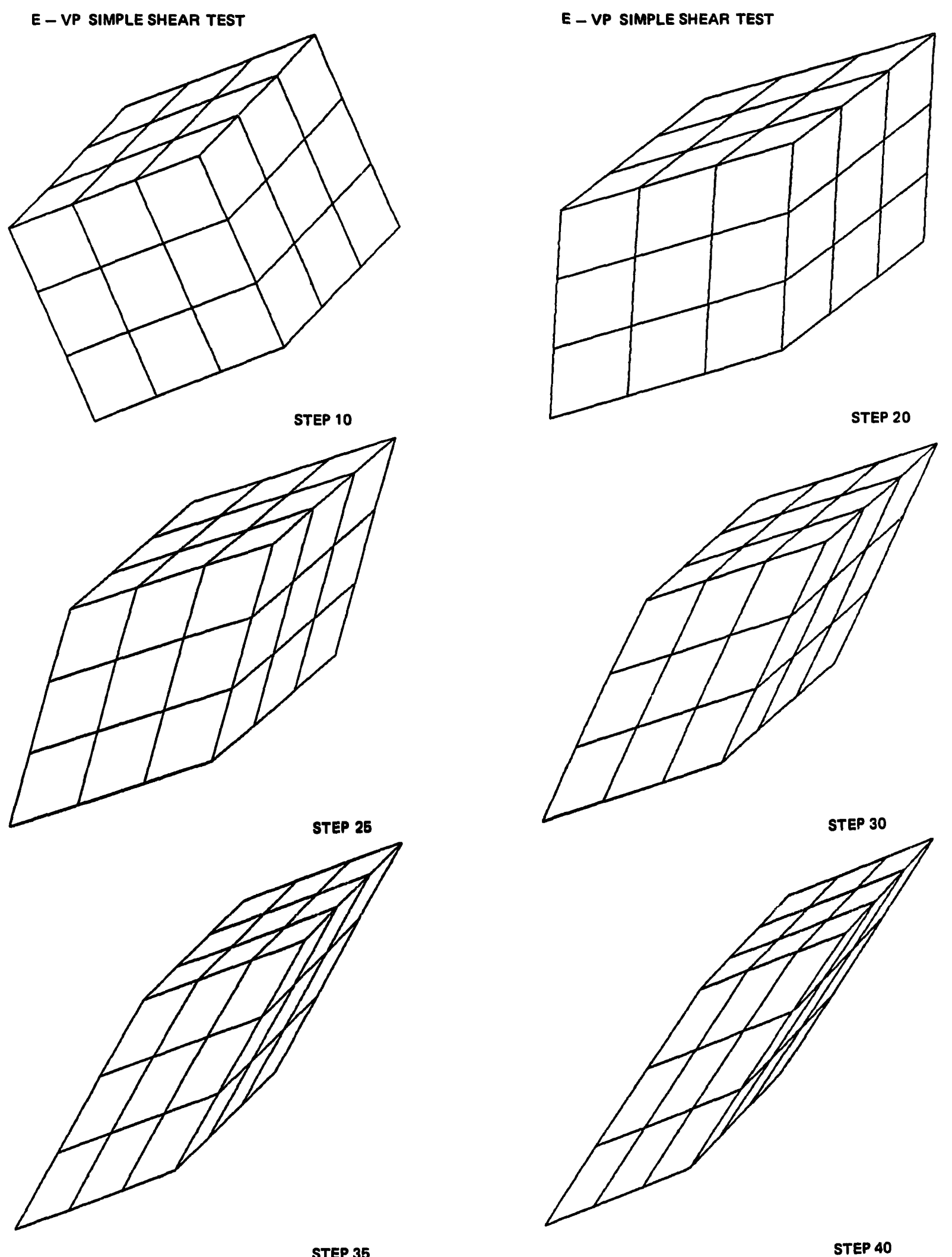

STEP 35

STEP 40

Fig. 12. Deformation patterns in simple shear test. 
the cube resulting in a very large shear strain (shearing angle is $63.4^{\circ}$ ). The material parameters used in this test are $E=10,000000 \mathrm{psi}, \nu=0.3, \sigma_{\mathrm{Y}}$ (initial yield stress) $=16000 \mathrm{psi}$, $E_{t}$ (elastic-plastic modulus) $=2307690 \mathrm{psi}, \gamma$ (viscosity parameter) $=0.001 \mathrm{sec}^{-1}$. Results obtained are shown in Fig. 11. The stress-strain plot indicates no oscillations whatsoever, and thus proves the effectiveniess of rotated Cauchy stress for large viscoplastic deformation. The results obtained by Key [44] are of the same nature as those obtained here.

EXAMPLE 3. Clamped and simply supported square plate. Various numerical experiments have been carried out with clamped and simply supported square plate problems. Only a quarter of the plate is modelled because of symmetry. The material parameters in this example are $E=10,000000 \mathrm{psi}, \nu=0.3, \sigma_{\mathrm{Y}}$ (initial yield stress) $=16000 \mathrm{psi}, E_{t}$ (elastic-plastic modulus) $=2307690 \mathrm{psi}, \gamma$ (viscosity parameter) $=0.001 \mathrm{sec}^{-1}$. The dimensions of the plate are 20 in $\times 20$ in $\times 0.4$ in. For the uniform pressure loading case where the pressure is incremented by 50 psi per step, the deformation patterns are shown in Figs. 15 and 19, respectively for the simply supported and clamped cases. Results obtained by full integration of the deviatoric parts of the stiffness and stress components, and those obtained by complete DRI were compared with the classical elastic series solution as shown in Figs. 14 and 18. It can be seen that in the elastic regime, the results obtained from DRI (geometry dependent integration points) are in much better agreement with the classical solution in comparison with the stiffer results obtained by full integration. Thin layer of boundary elements as shown in Figs. 15 and 19 are introduced in order to avoid any hourglassing that may arise because of the boundary layer effect. This has been explained clearly in [42]. Figures 14 and 18 clearly illustrate the transition from flexure to membrane action that is expected in plate analysis. The deflection along the line of symmetry as the load increases is demonstrated in Figs. 13 and 17. Figures 16

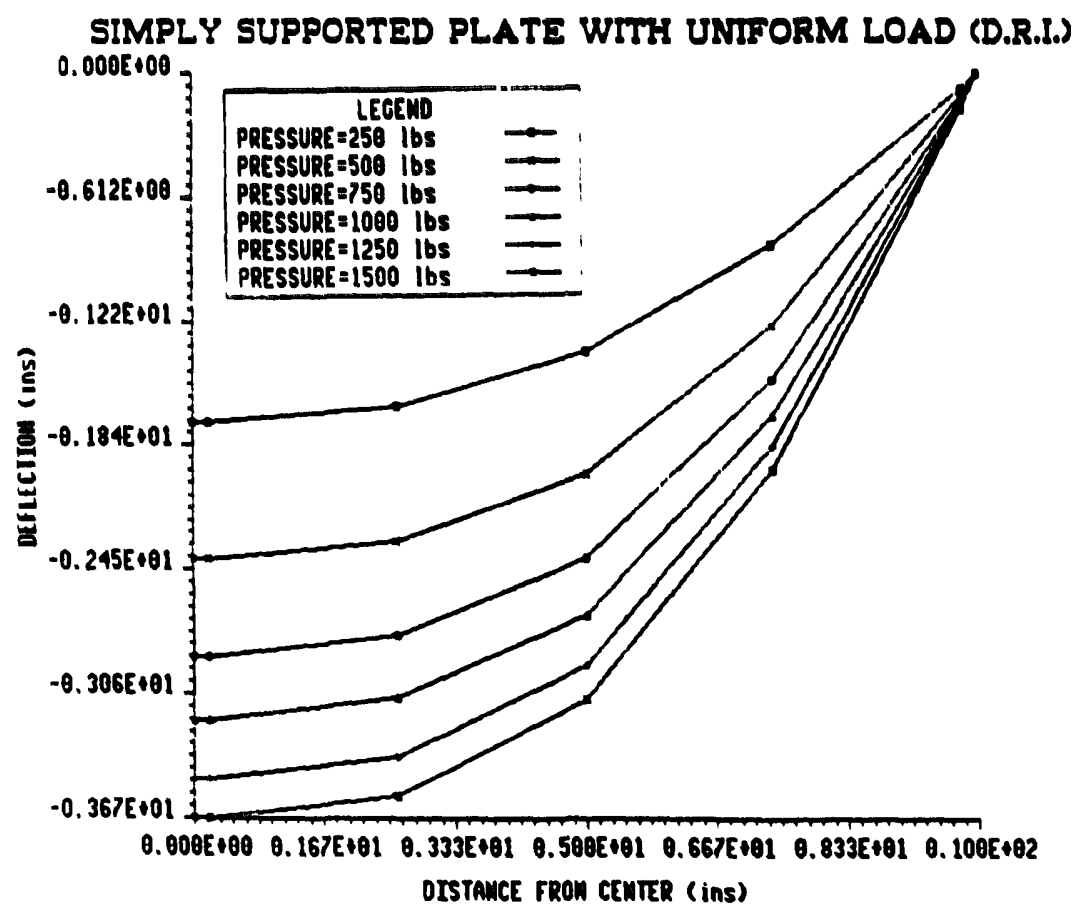

Fig. 13. Deflection (in) along the centerline of a simply supported plate under uniform load. 


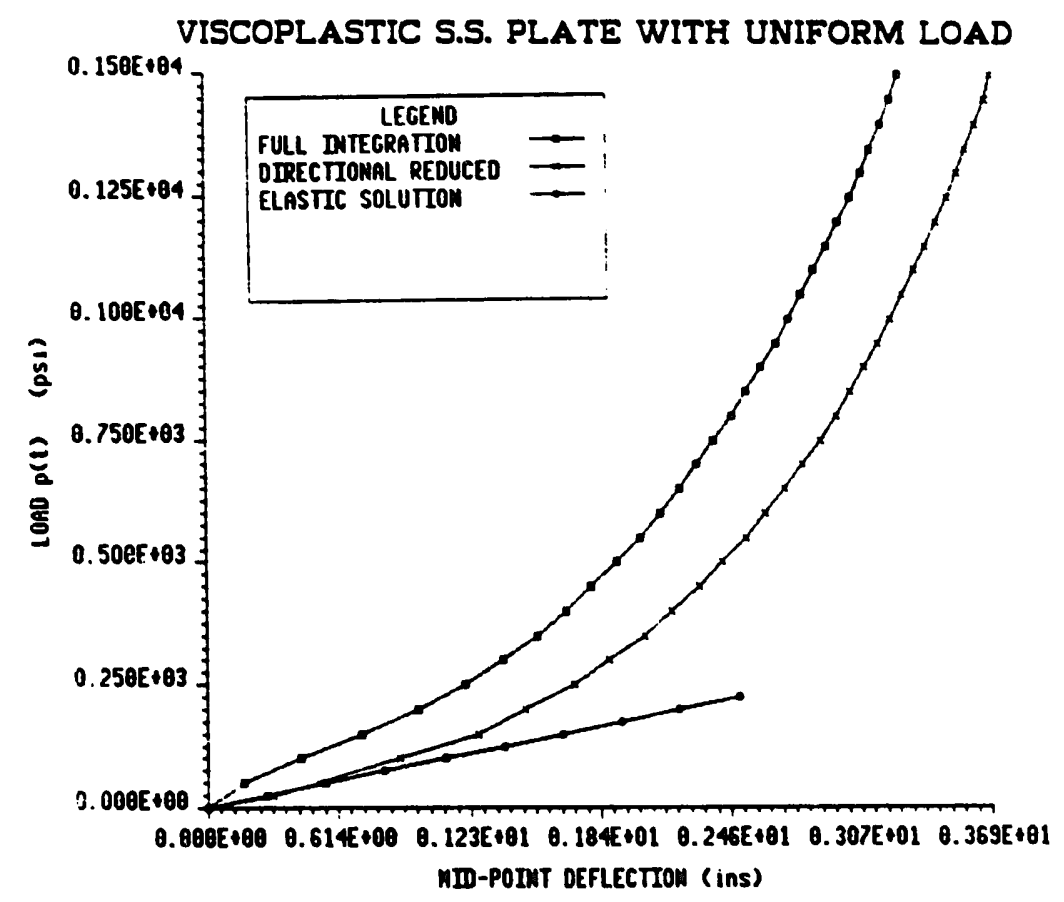

Fig. 14. Applied pressure (psi) versus mid-point deflection (in) for the simply supported plate.

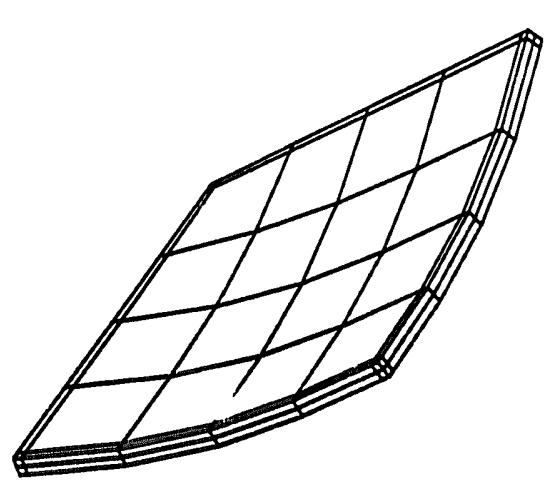

STEP NUMBER 5

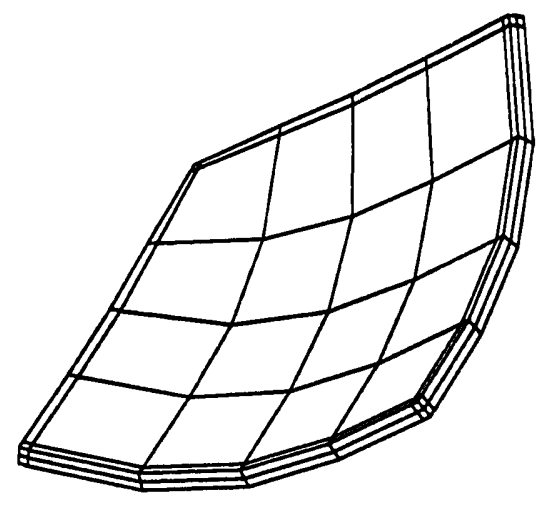

STEP NUMBER 20

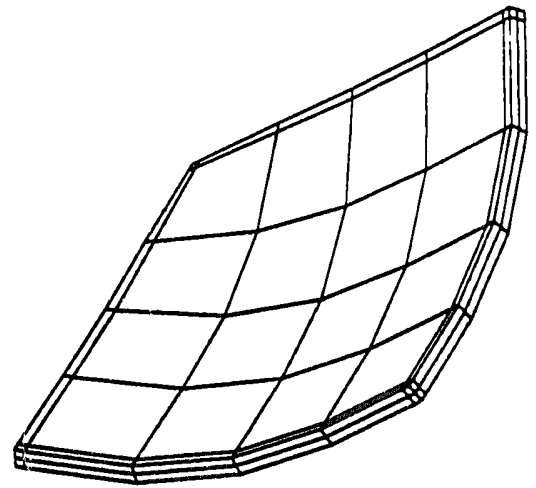

STEP NUMBER 15

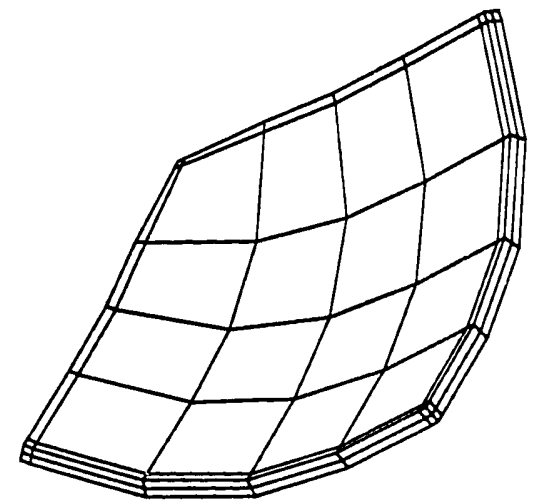

STEP NUMBER 30

Fig. 15. Deformation patterns of a simply supported plate under uniform loading. 


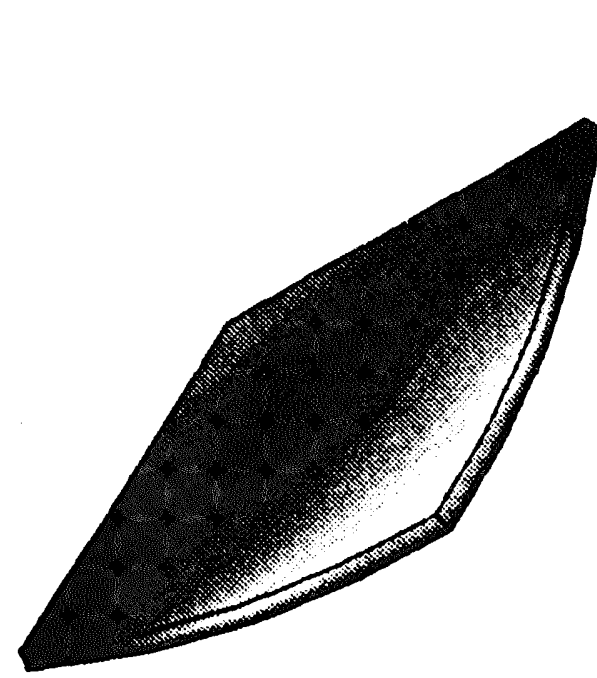

STEP NUMBER 5

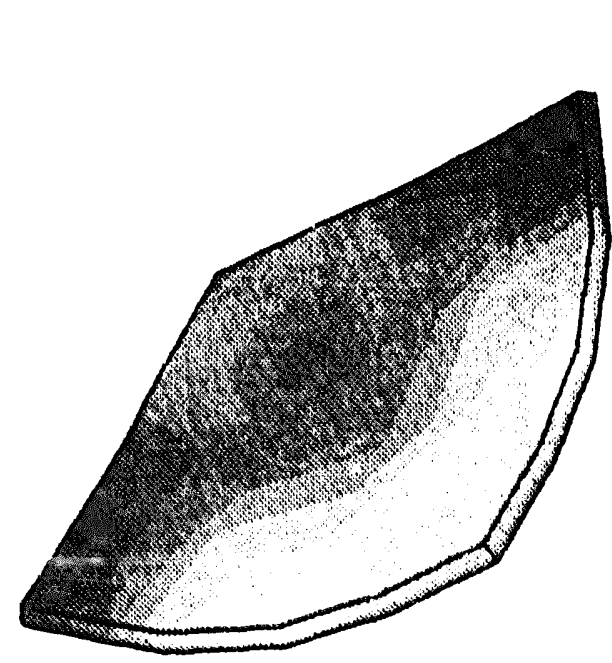

STEP NUMBER 20
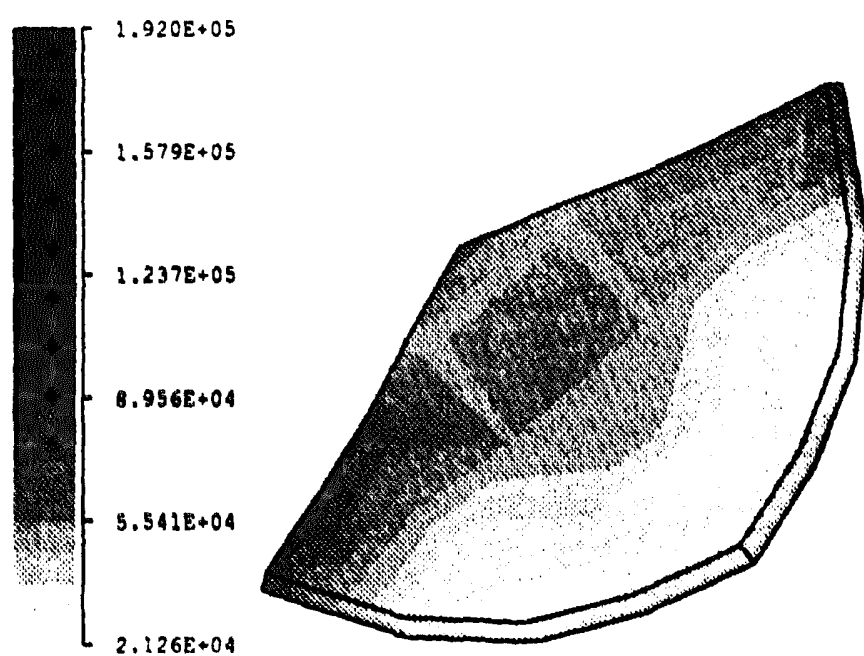

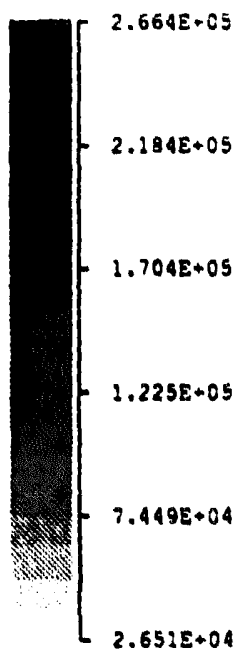

STEP NUMBER 30

Fig. 16. Equivalent stress contours (psi) in a simply supported plate under uniform loading.

and 20 show the equivalent stress contours for uniformly loaded simply supported plate and clamped plate, respectively.

Similar numerical experiments were conducted with simply supported and clamped plates under concentrated central loading. The load $P(t)$ was, in these cases, incremented by $1000 \mathrm{lbs}$ per time step. Consistent with the observations for uniform loading, the response with full integration was stiffer than that with DRI, though in this case the pure elastic solution was 


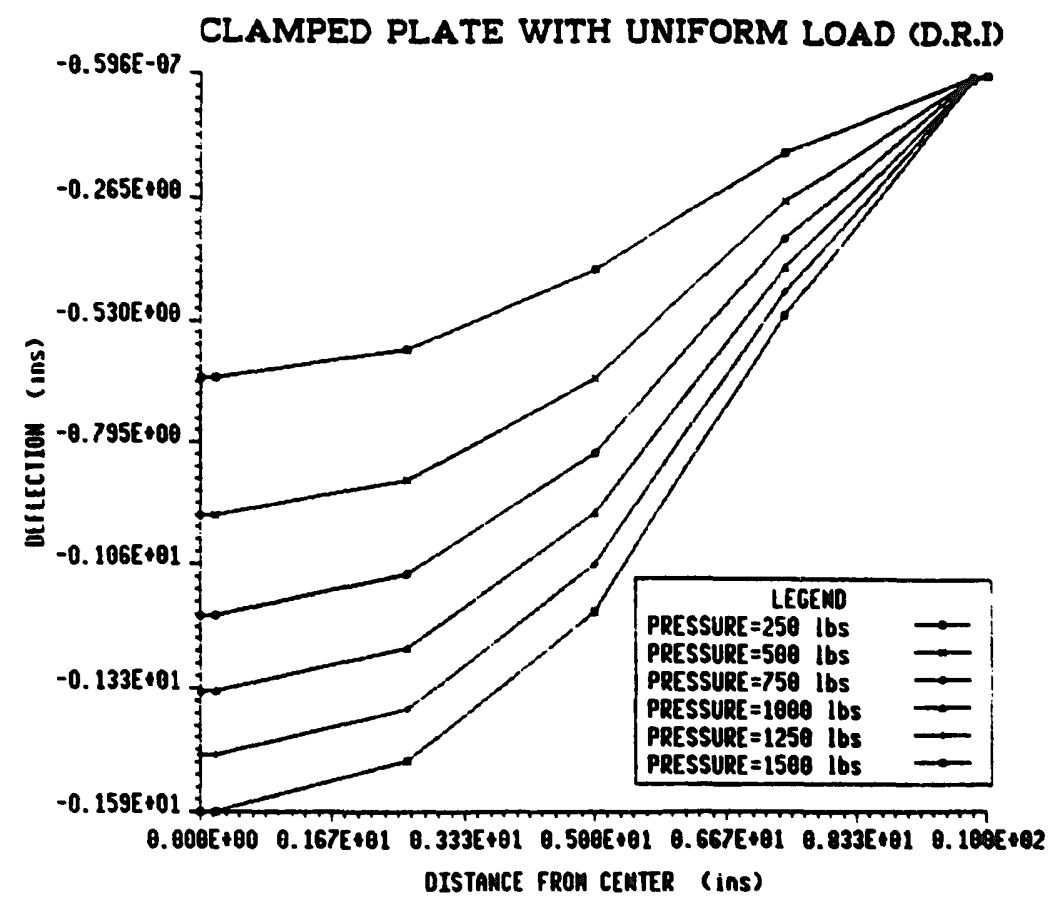

Fig. 17. Deflection (in) along the centerline of a clamped plate under uniform load.

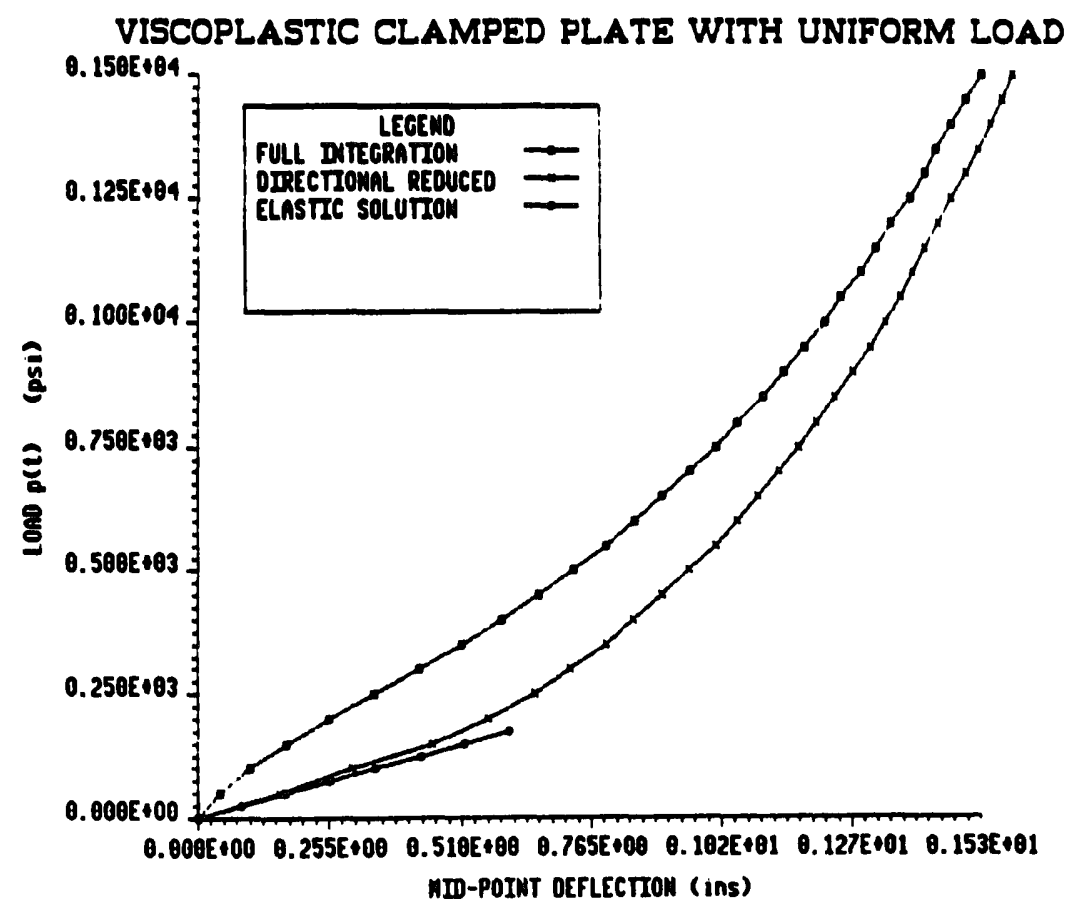

Fig. 18. Applied pressure (psi) versus mid-point deflection (in) for the clamped plate. 


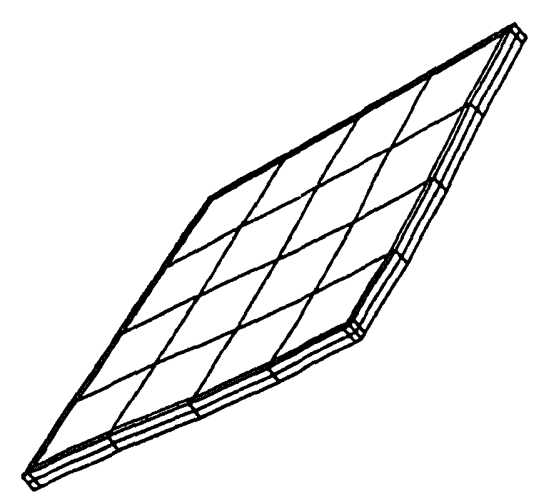

STEP NUMBER 5

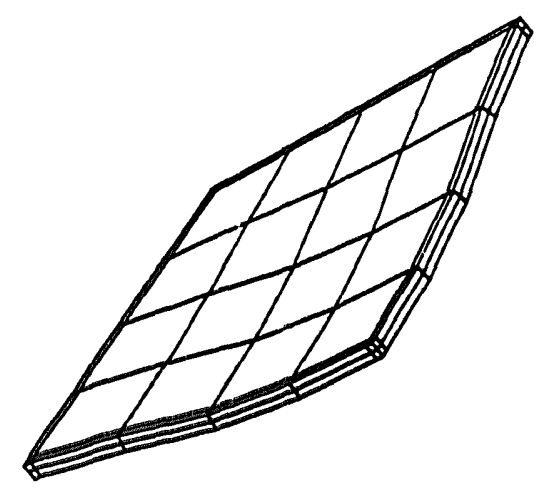

STEP NUMBER 20

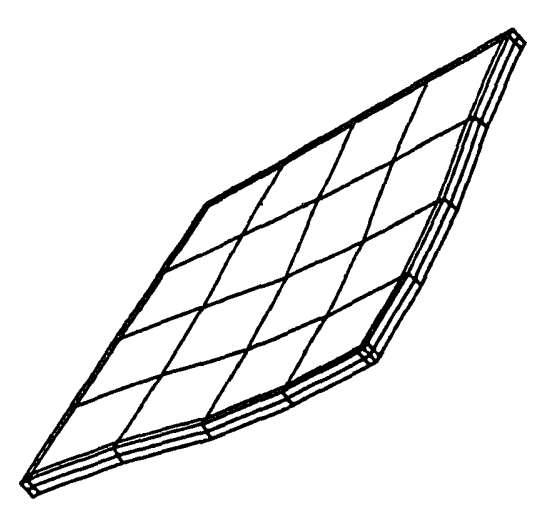

STEP NUMBER 15

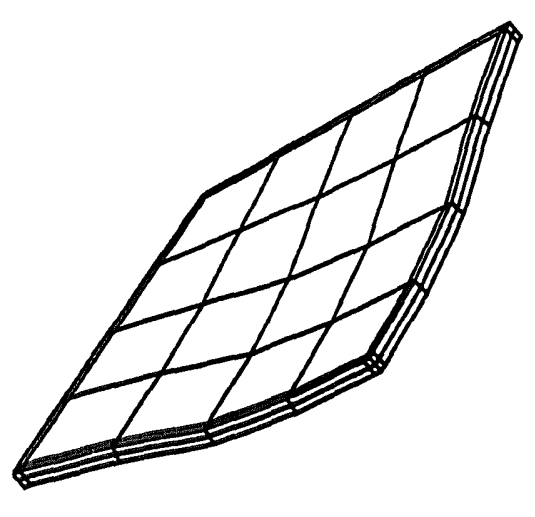

STEP NUMBER 30

Fig. 19. Deformation patterns of a clamped plate under uniform loading.

somewhat stiffer than the latter. This response can be seen from Fig. 26, where the center deflection is plotted as a function of the load. The load causes viscoplastic yielding even in the first step and hence the elastic solution is omitted. Figures 21 and 22 show the deformation patterns and equivalent stress contours for the simply supported plate, while Figs. 23 and 24 show the same for the clamped plate. In Fig. 25 the deflection curves of the symmetry lines for the clamped plate are presented.

\subsection{Metal forming type problems with ALE description}

EXAMPLE 4. Bending of variable length beam. In this case, a rectangular beam in a guide, which is being pushed out by a piston from one side and is acted upon by a variable concentrated load $P(t)$ at the other, as shown in Fig. 27 is analyzed. 


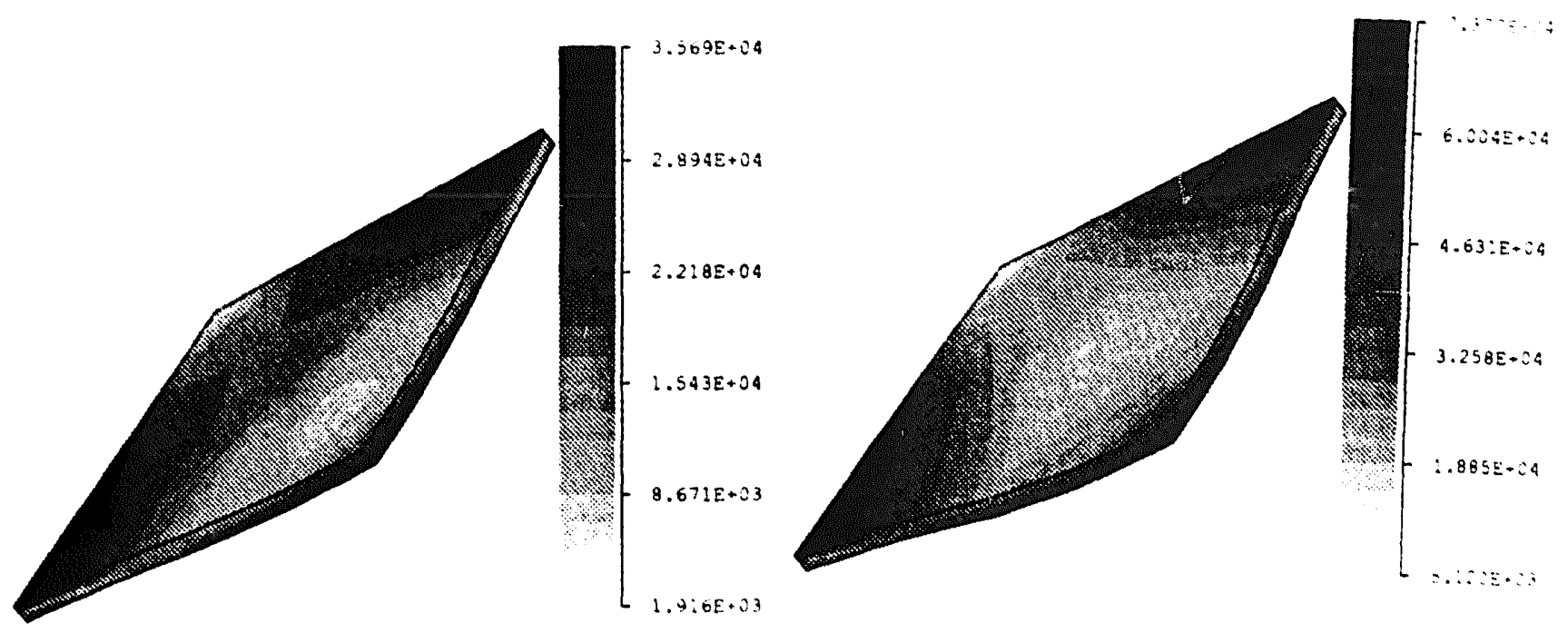

STEP NUMBER 5

STEP NUMBER 15

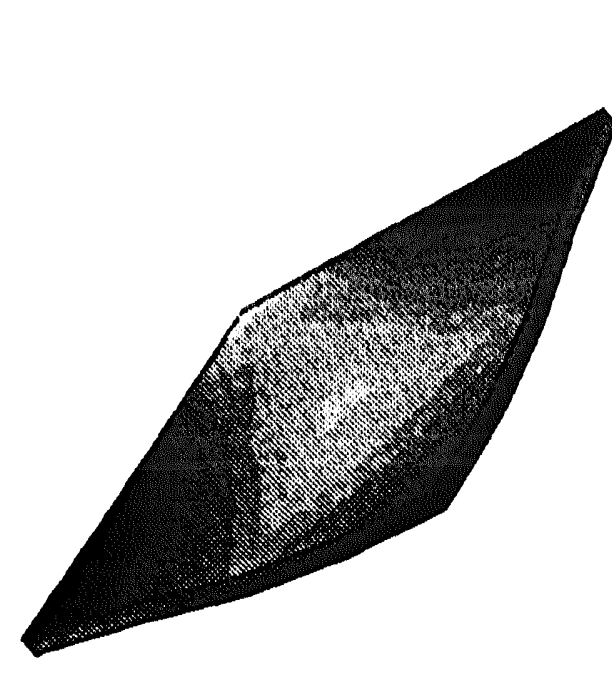

STEP NUMBER 20
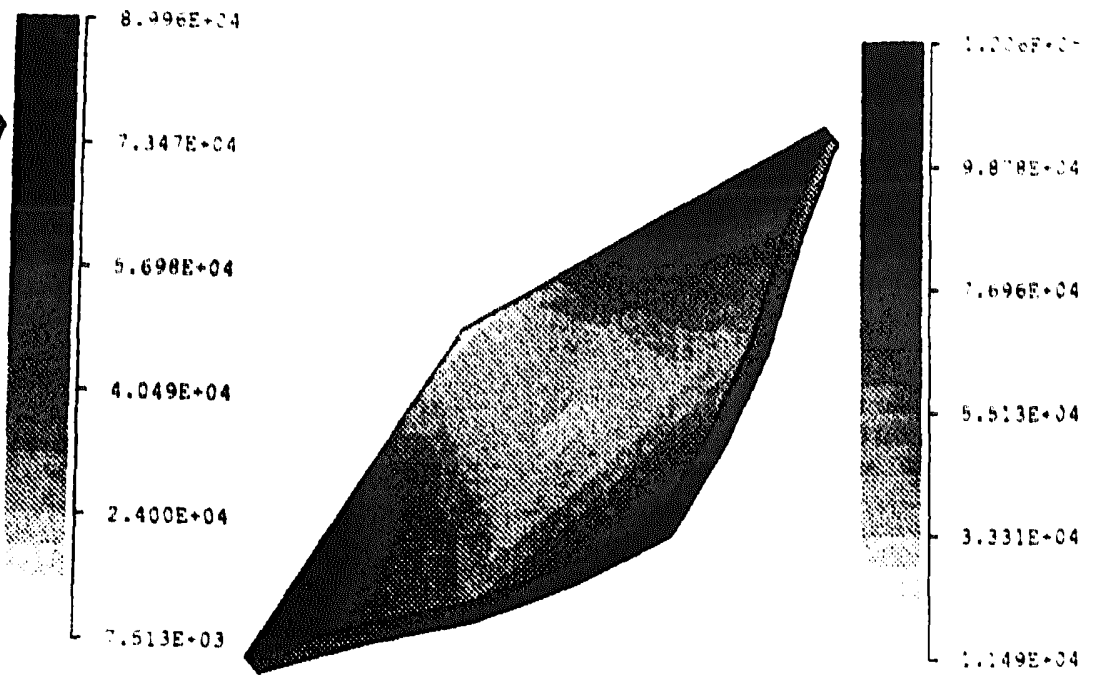

Fig. 20. Equivalent stress contours (psi) in a clamped plate under uniform loading. 


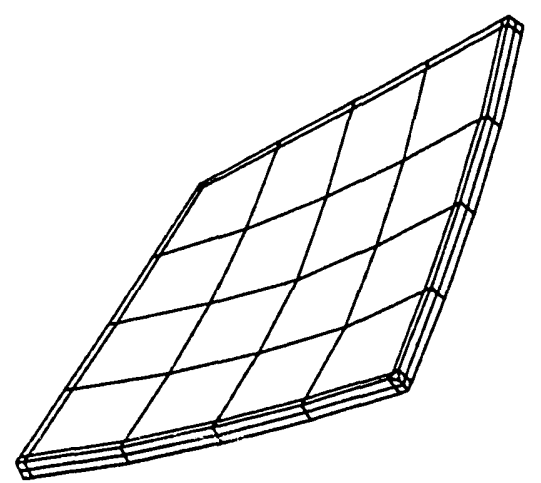

STEP NUMBER $=15$

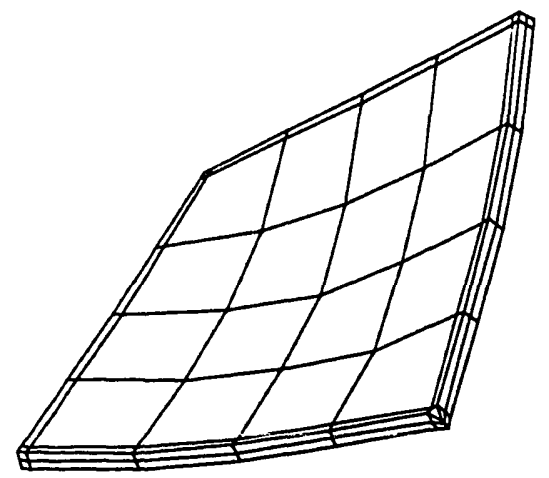

STEP NUMBER $=30$

Fig. 21. Deformation of a simply supported plate under concentrated load.

The material parameters in this example are $E=10,000000 \mathrm{psi}, \nu=0.3, \sigma_{\mathrm{Y}}$ (initial yield stress) $=16000 \mathrm{psi}, \quad E_{t}$ (elastic-plastic modulus) $=2307690 \mathrm{psi}, \gamma$ (viscosity parameter) $=$ $0.001 \mathrm{sec}^{-1}$. The load is incremented by $152 \mathrm{lbs}$ in each time step and the velocity of the piston is maintained at $0.005 \mathrm{in} / \mathrm{sec}$. The analysis is continued over a time period of $0.3 \mathrm{sec}$ in thirty equal time steps. The load increment is stopped after twenty five steps. The typical Lagrangian approach fails to represent the precise effect of the sharp edges of the guide in this example. This is because the displacement boundary conditions are specified on the material points which may physically move away from the edges. It is here that the flexibility of the ALE description may be used favorably. The nodes coinciding with the edge of the guide are held

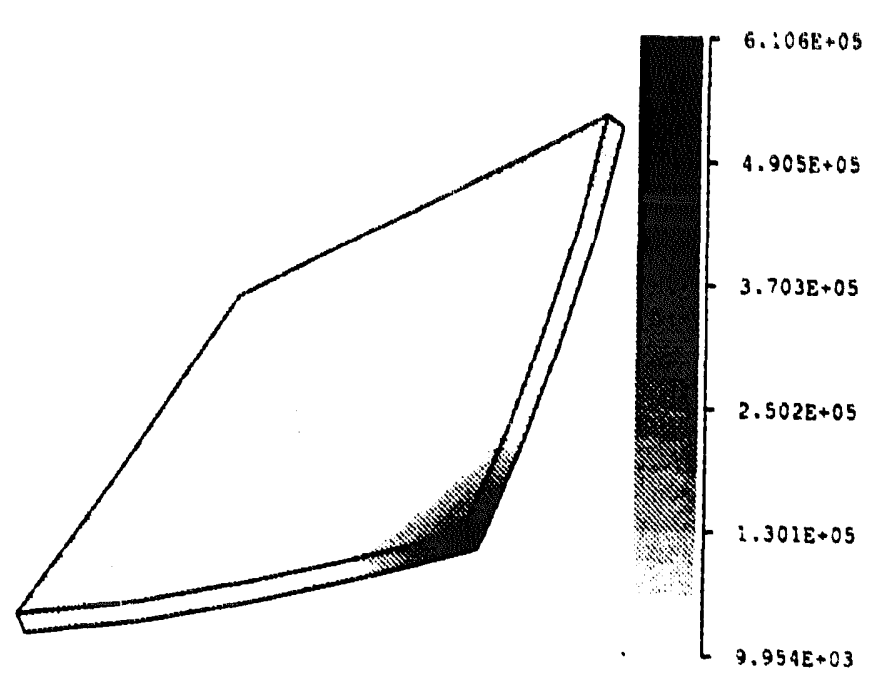

STEP NUMBER $=15$

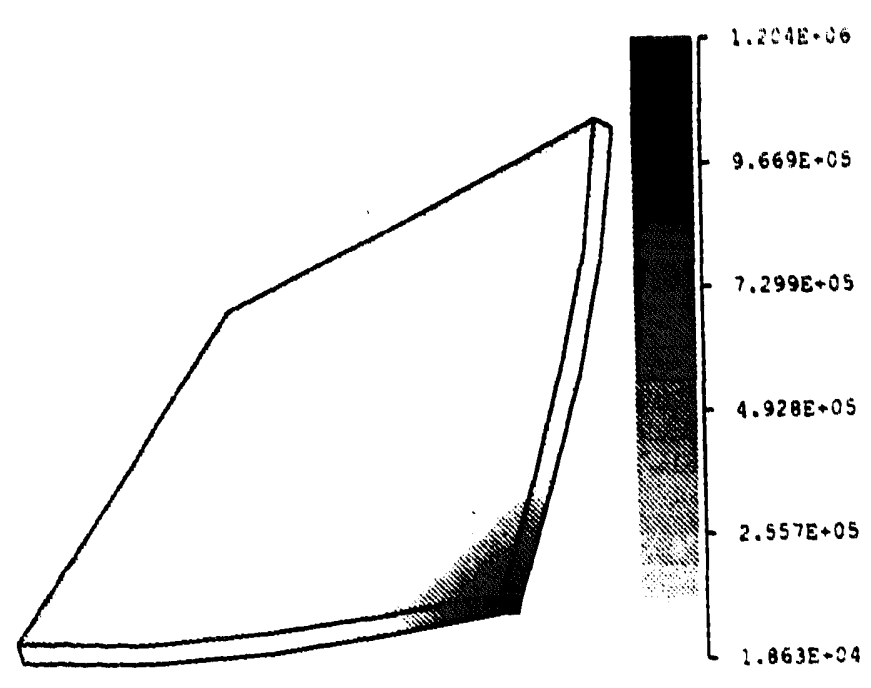

STEP NUMBER $=30$

Fig. 22. Equivalent stress contours (psi) of a simply supported plate under concentrated load. 


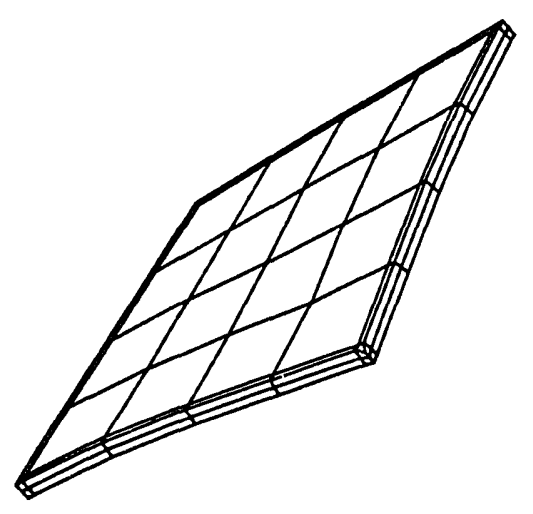

STEP NUMBER $=15$

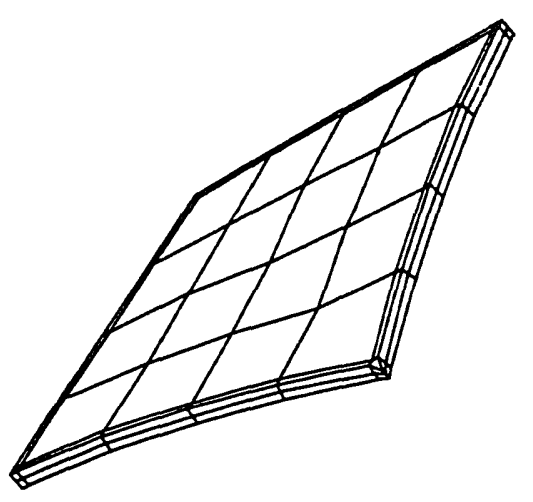

STEP NUMBER $=30$

Fig. 23. Deformation of a clamped plate under concentrated load.

fixed (Eulerian) and the surface nodes under the guide are moved towards the edge with specified velocities. The latter movement is monitored to better represent the stress jump across the edges. A comparative study with full integration and DRI $(2 \times 1 \times 2)$ schemes is presented in Figs. 28 and 30, which indicate the variation of flexural stress along the beam and the end deflection with time, respectively. It is noticed from Fig. 29 that the relaxation effects become pronounced with time especially at the free end of the beam. Figure 31 shows the deformation patterns of the beam for a few selected time steps and Fig. 32 shows the flexural stress contours.
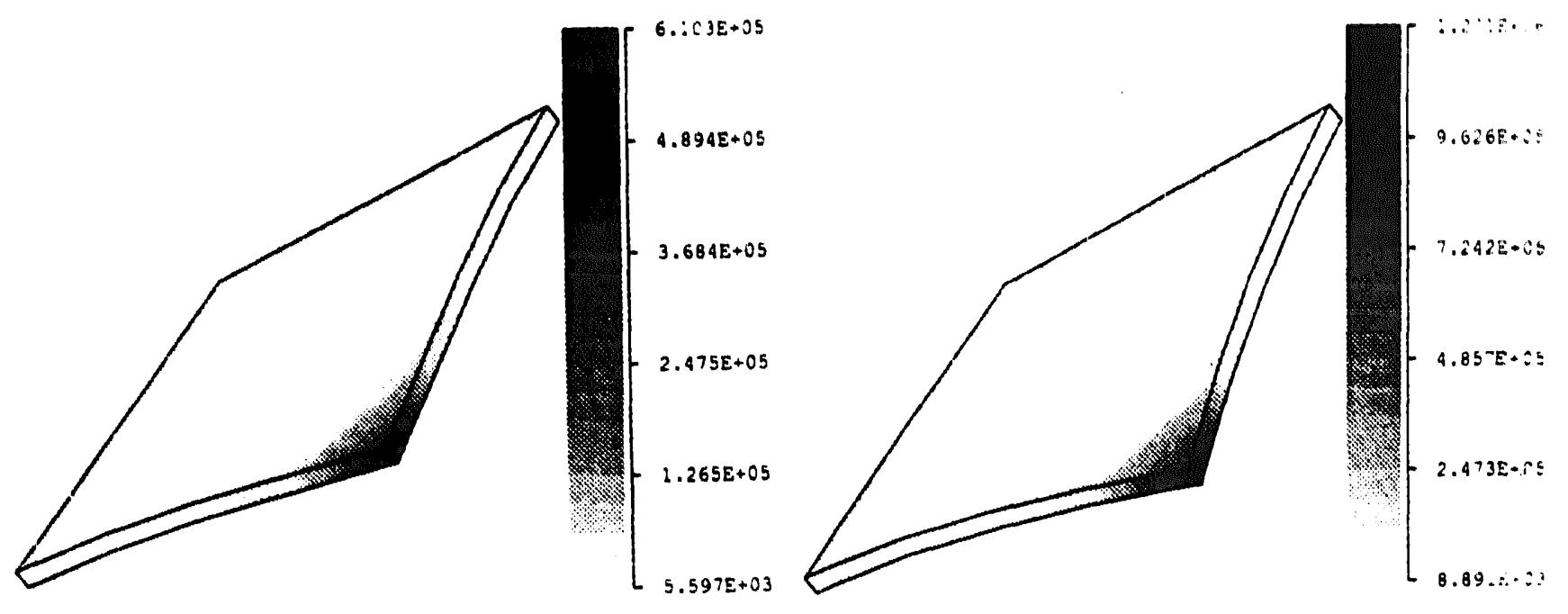

STEP NUMBER $=15$

STEP NUMBER $=30$

Fig. 24. Equivalent stress contours (psi) of a clampled plate under concentrated load. 


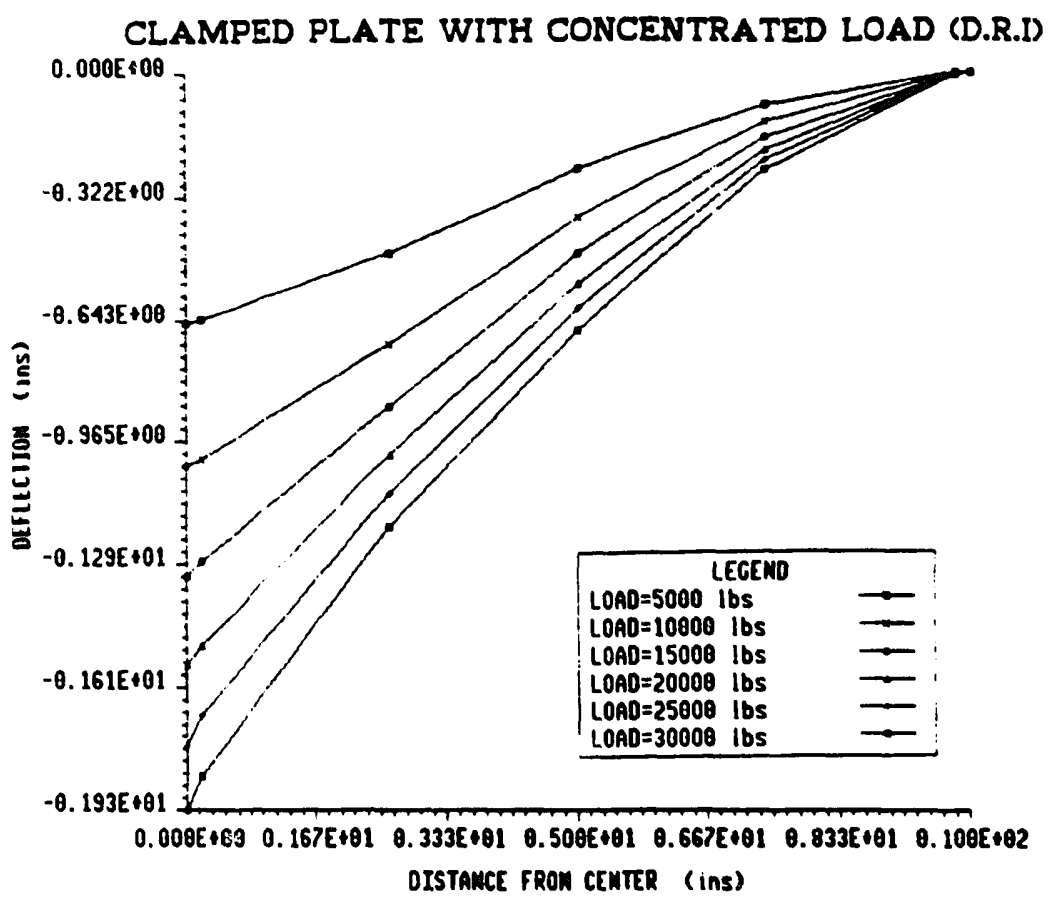

Fig. 25. Deflection (in) along the centerline of a clamped plate under concentrated load.

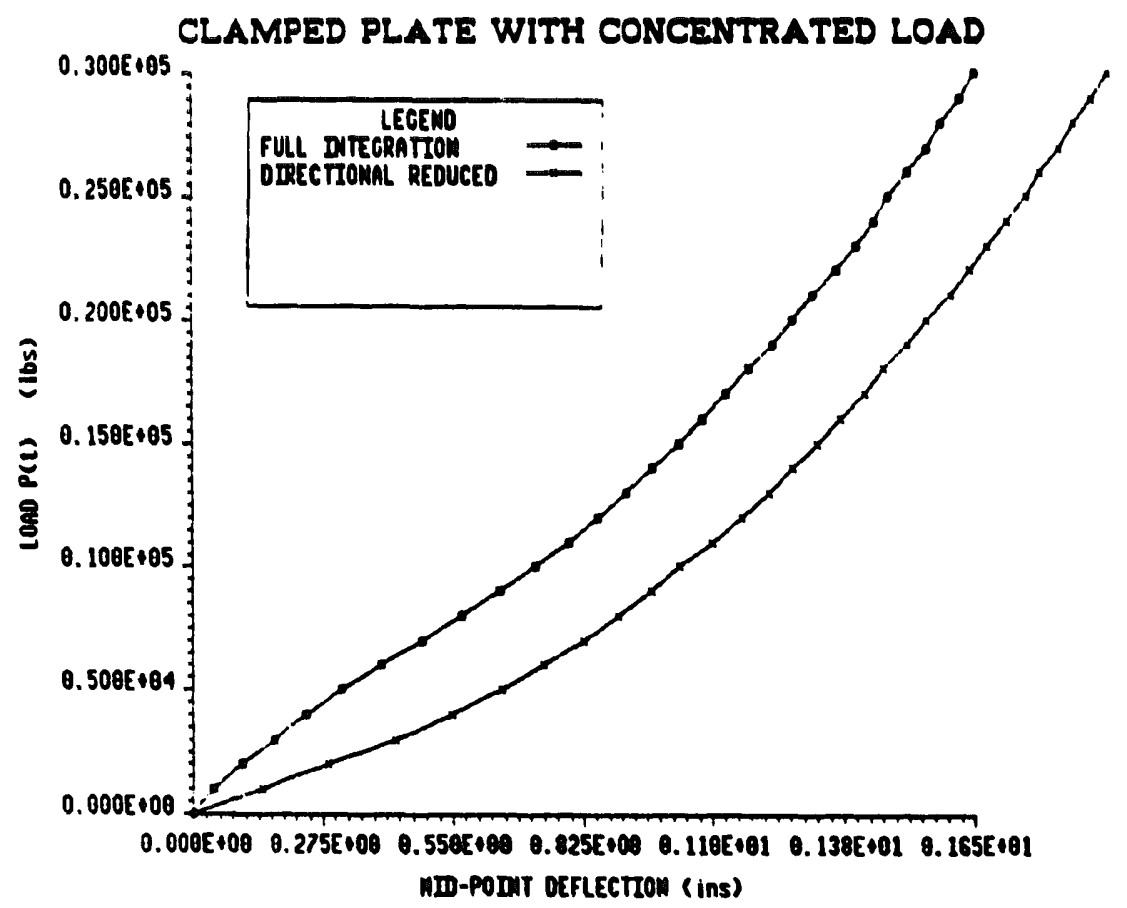

Fig. 26. Applied concentrated load (lbs) versus mid-point deflection (in) for the clamped plate. 
Young's Modulus $=3000000$ psi

Poisson Ratio $=0.3 \quad 3$

Yield stress $\quad-16 \times 10$ psi

Viscosity parameter $=0.001 \mathrm{sec}$

Piston velocity $\quad=0.005$ ins $/ \mathrm{sec}$

Force $P(t) \quad-152 n$ lbs

$n=\operatorname{siep~no.~}$

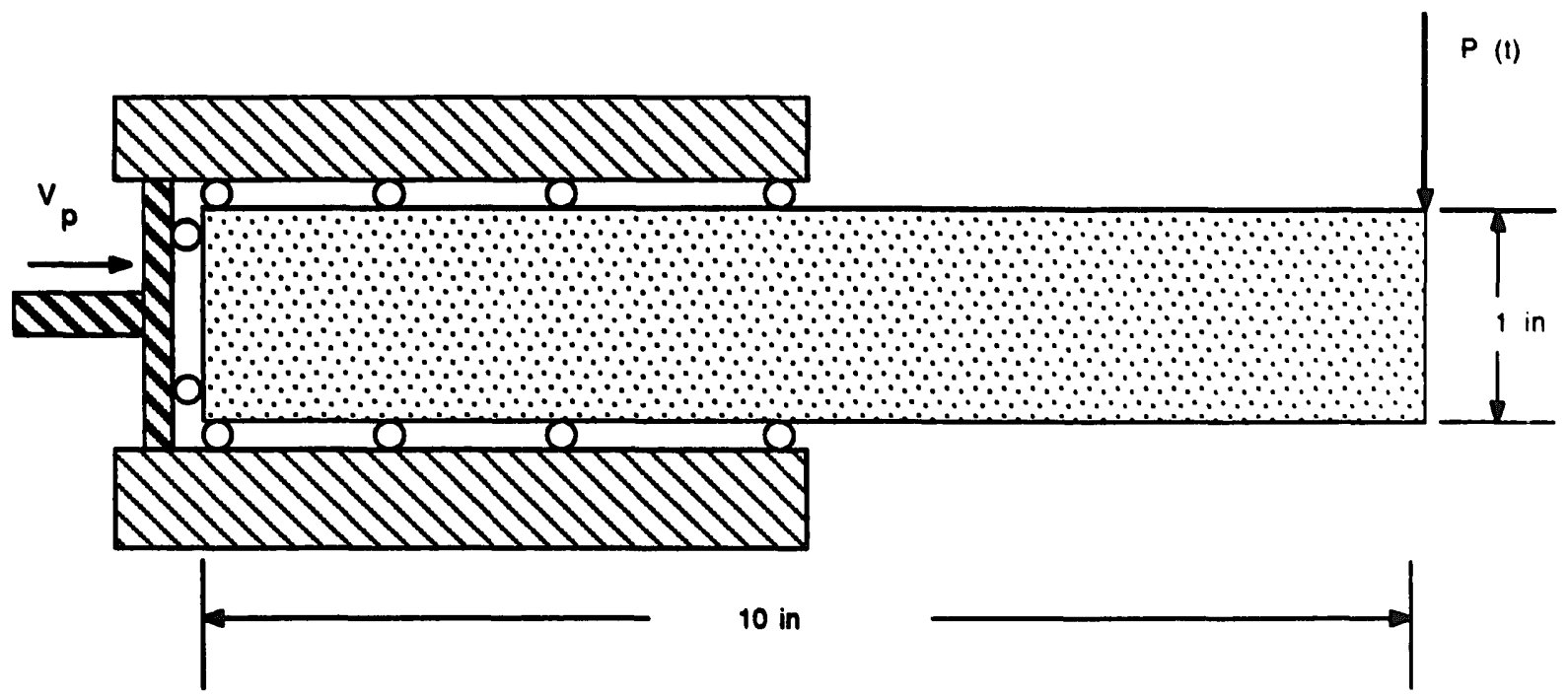

Fig. 27. Section view of 3-D beam pushed by a piston at one end and loaded by a variable concentrated force at the other end.

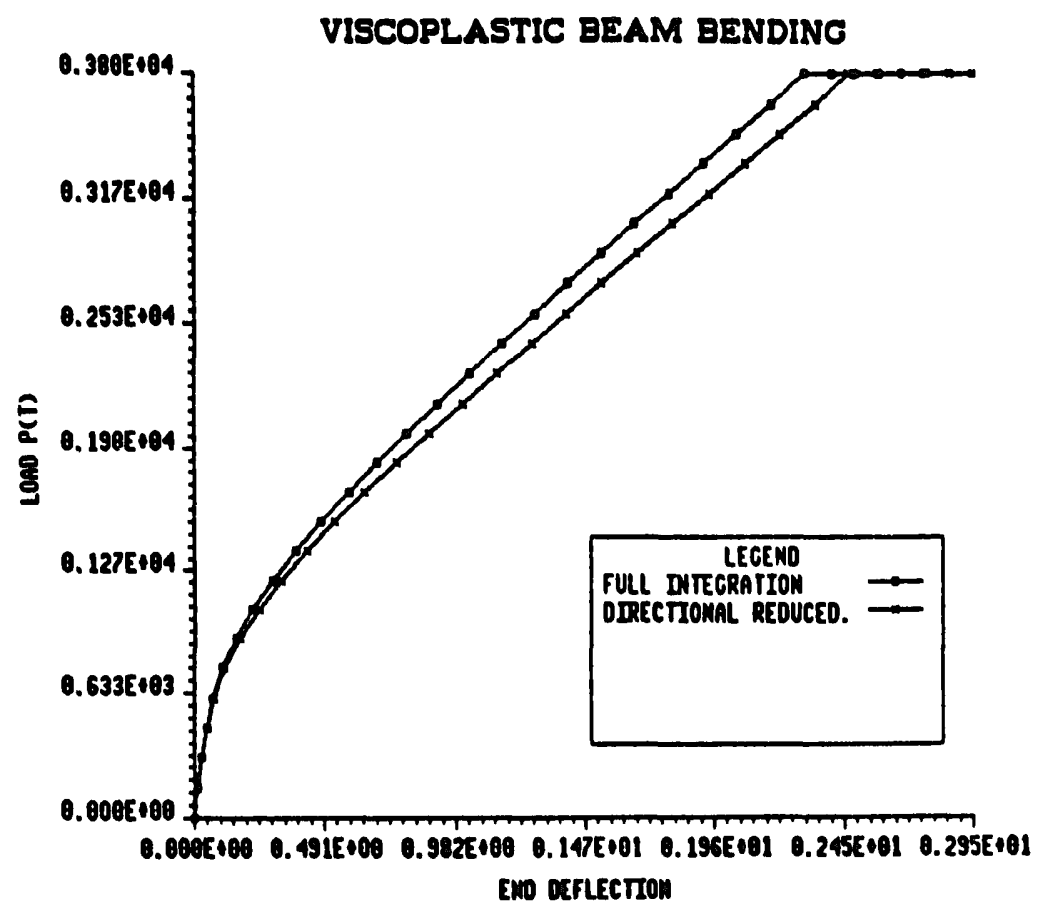

Fig. 28. Load (lbs) versus end deflection (in) in viscoplastic beam bending with relaxation. 


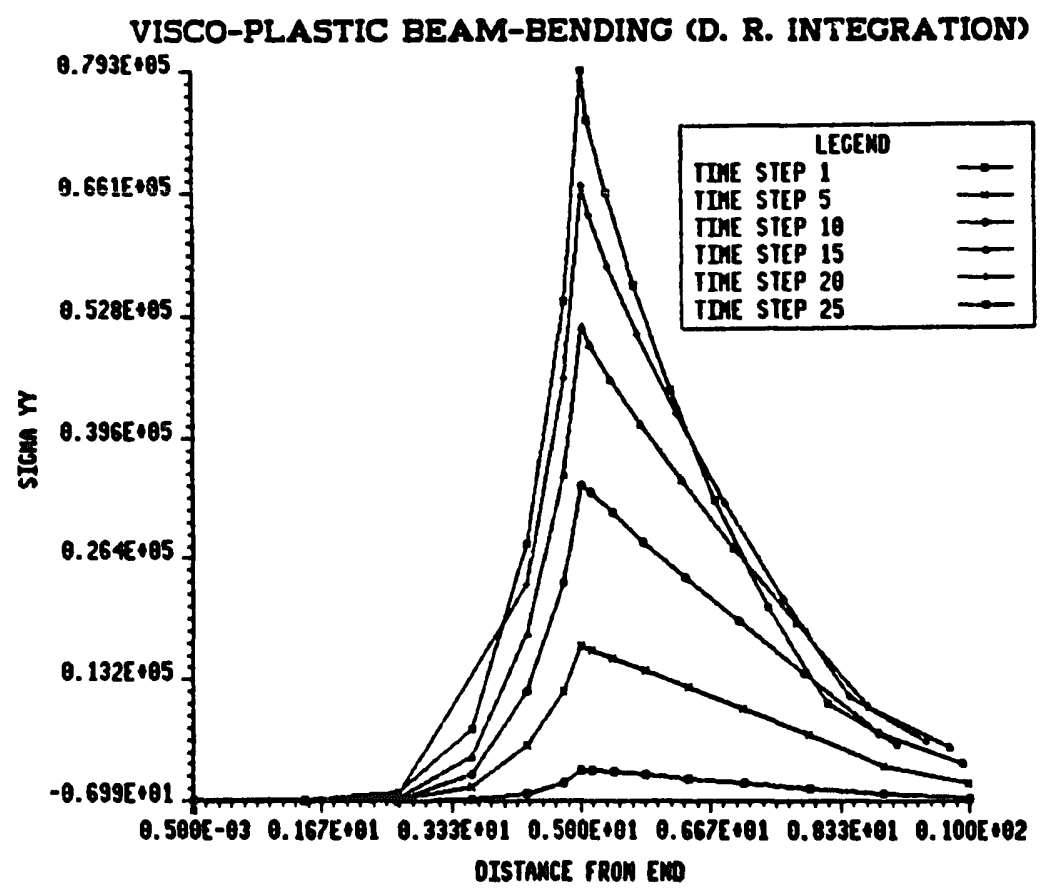

Fig. 29. Flexural stress (psi) versus distance from one end (in) in beam bending.

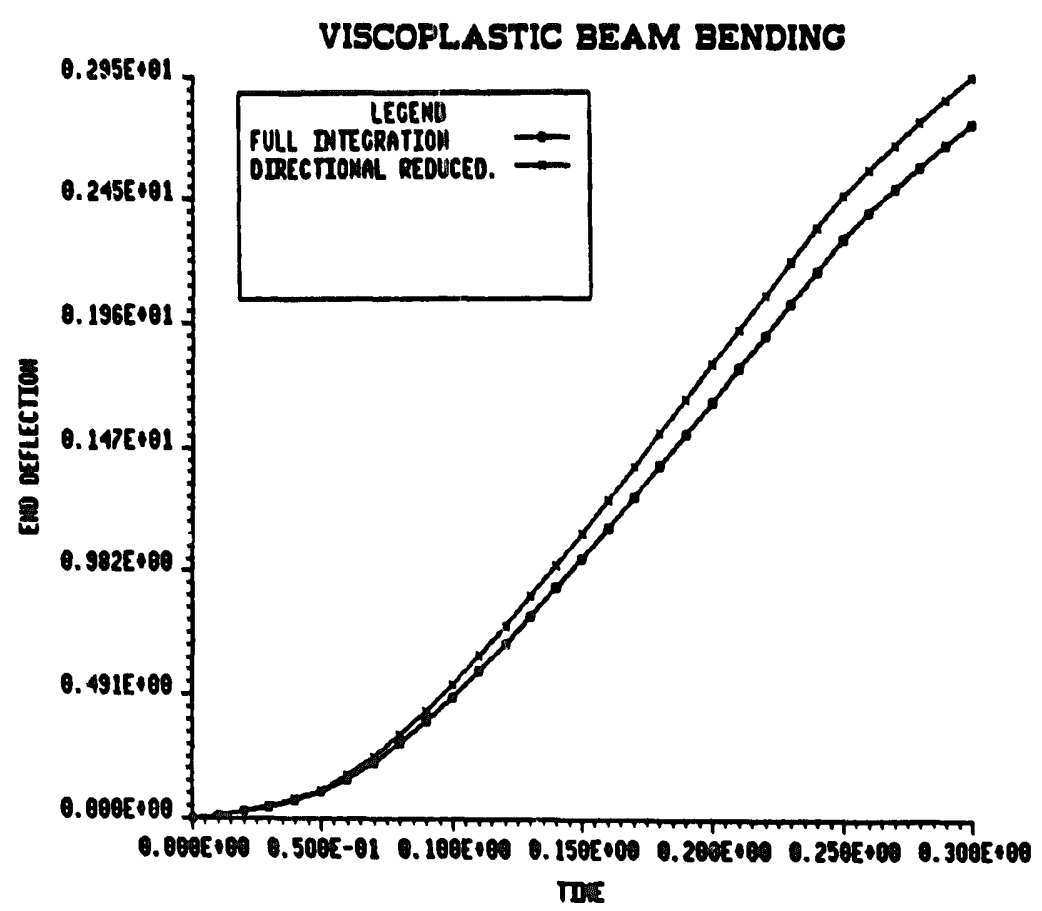

Fig. 30. End deflection (in) versus time (sec) in beam bending. 

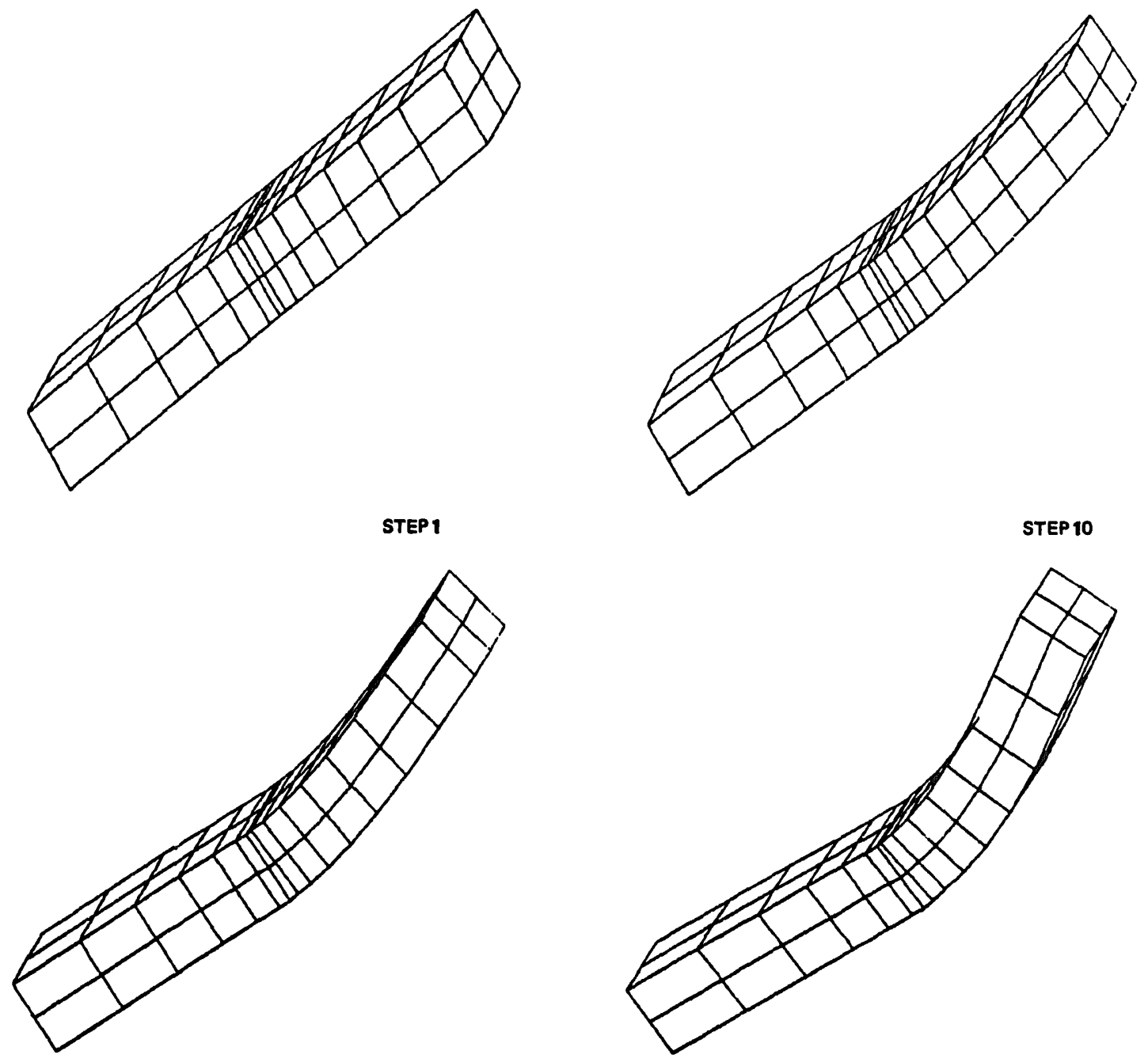

STEP 15

STEP 20
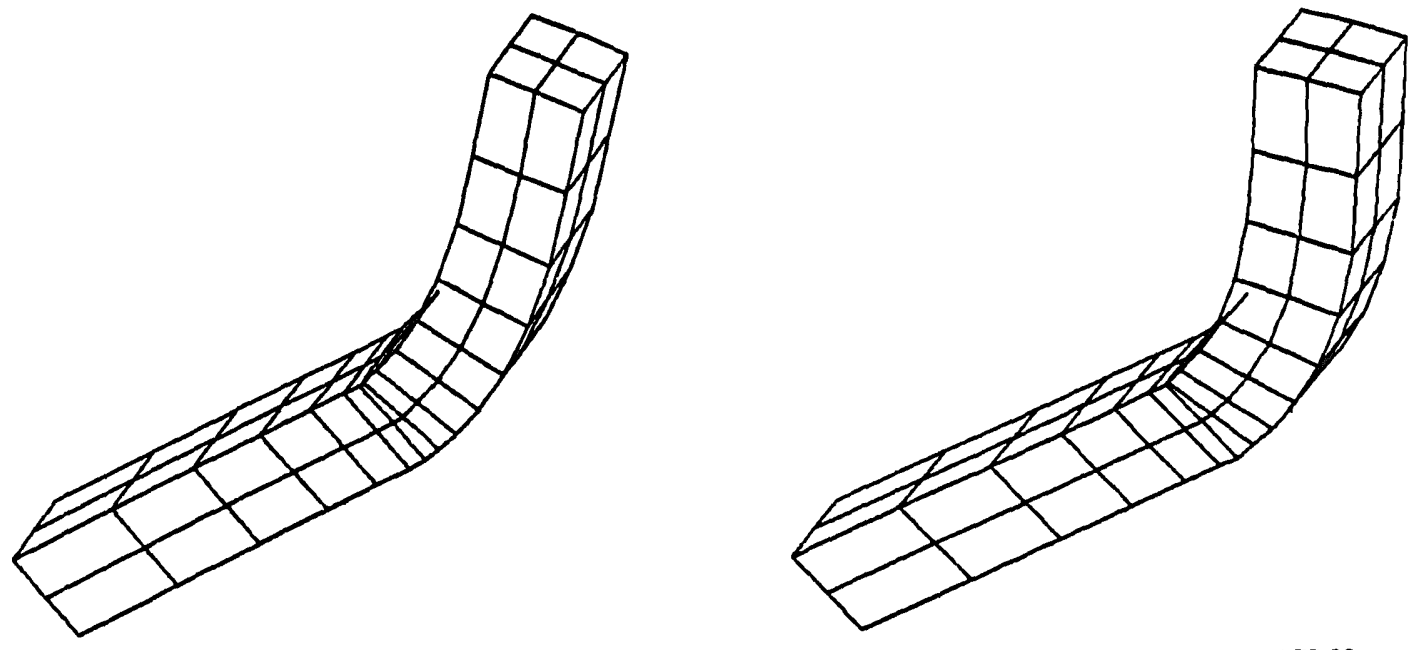

STEP 25

STEP 30

Fig. 31. Deformation patterns for beam bending. 


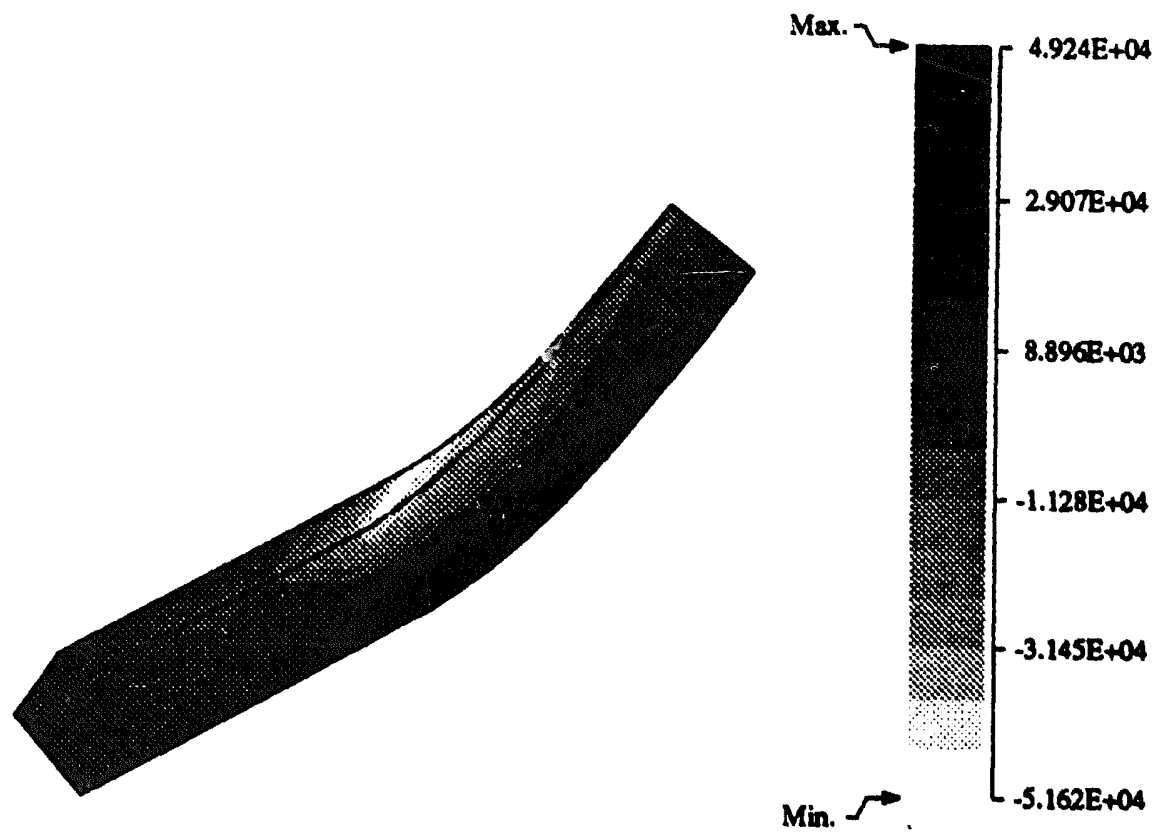

STEP NUMBER $=15$
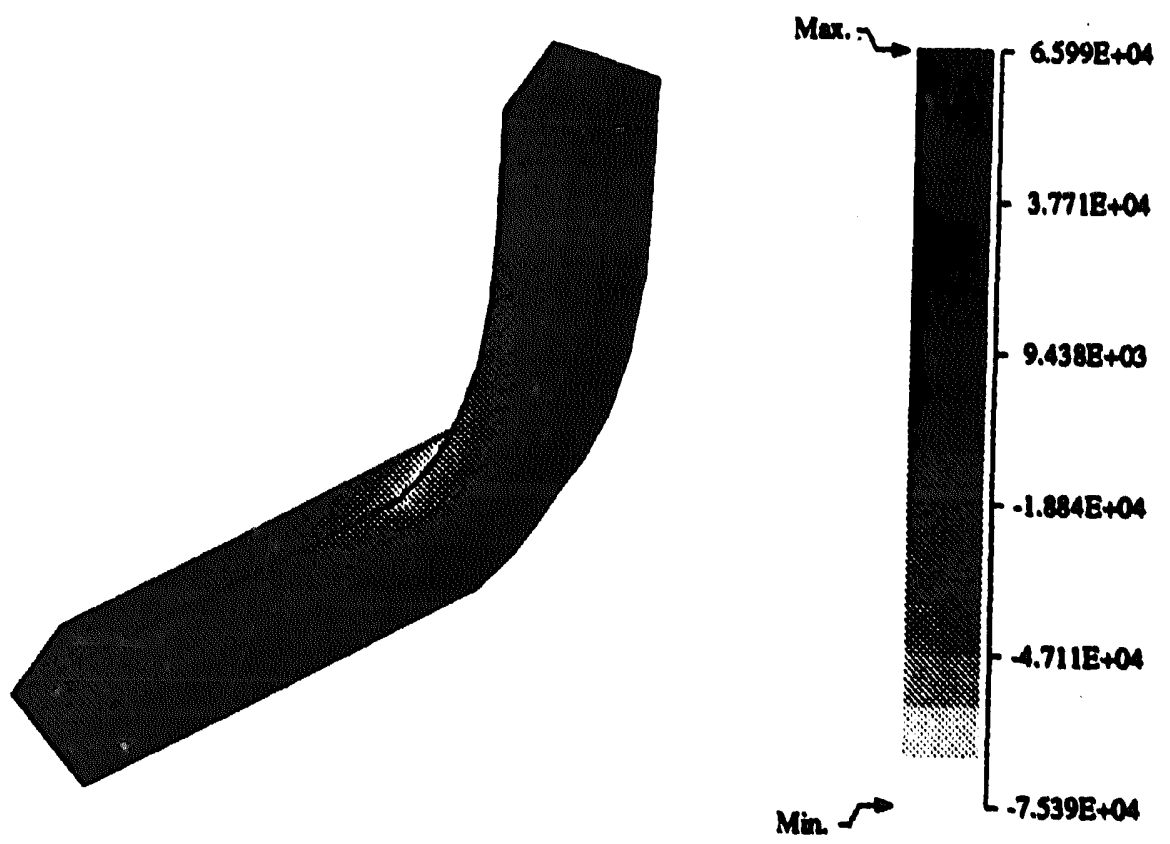

STEP NUMBER $=30$

Fig. 32. Flexural stress contours (psi) in beam bending problem. 
EXAMPLE 5. Indentation by a semi-cylindrical rigid punch. This example is aimed at testing the contact algorithms in the context of large deformation. A semi-cylindrical frictionless punch is made to indent an elastic-viscoplastic slab with a constant velocity of $15 \mathrm{in} / \mathrm{sec}$. The dimensions of the punch and the slab and the material constants are given in Fig. 33. The movement of the punch is restrained in the transverse direction by introducing frictionless walls. Only the right half of the slab is analyzed because of symmetry. The process is carried out for a period of $0.067 \mathrm{sec}$, during which time the punch progressively sinks through approximately its own radius. Owing to the absence of any sharp discontinuities in boundary conditions, the nodes in this analysis are made to be Lagrangian. Of course, in the event that the elements may become squeezed due to sudden impact by the punch, ALE nodes may have to be created under the punch. However, such circumstances have been avoided in the present work and hence ordinary Lagrangian nodes suffice.

The computed results of the analysis are compared with the elastic-plastic counterpart performed by Dumas and Baronet [45]. Deformation profiles at various stages of the process can be seen from Fig. 34. The extent of the viscoplastic zone propagation, which originate inside the material under the punch tip, is effectively observed from the shades of equivalent stress contours in Fig. 35 and these in good harmony with the schematic representation of plastic zones in [45]. Figure 36 is a depiction of the pressure profiles in the contacting zone as a function of the angle made by the line joining the center of the punch of the contact node with

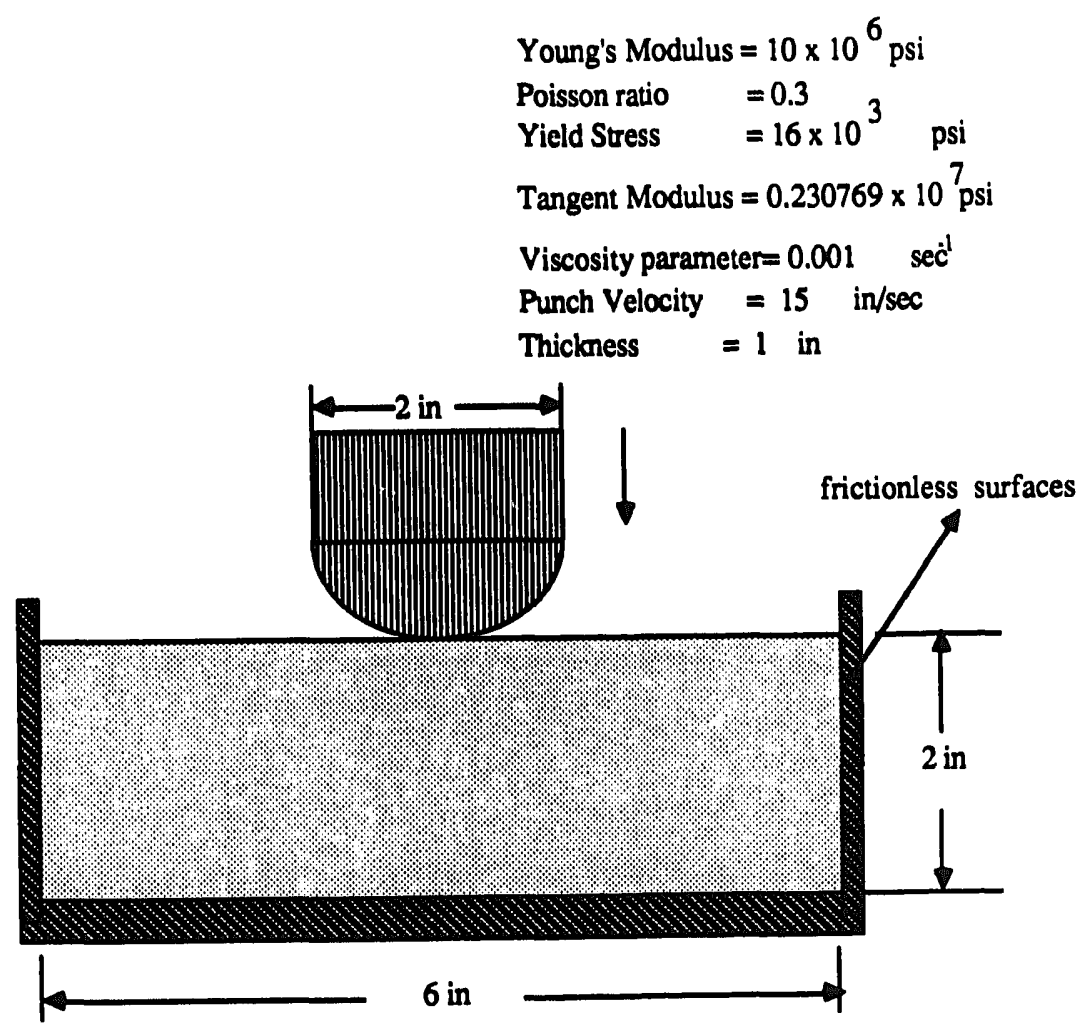

Fig. 33. Section view of indentation of a solid block by a rigid punch. 


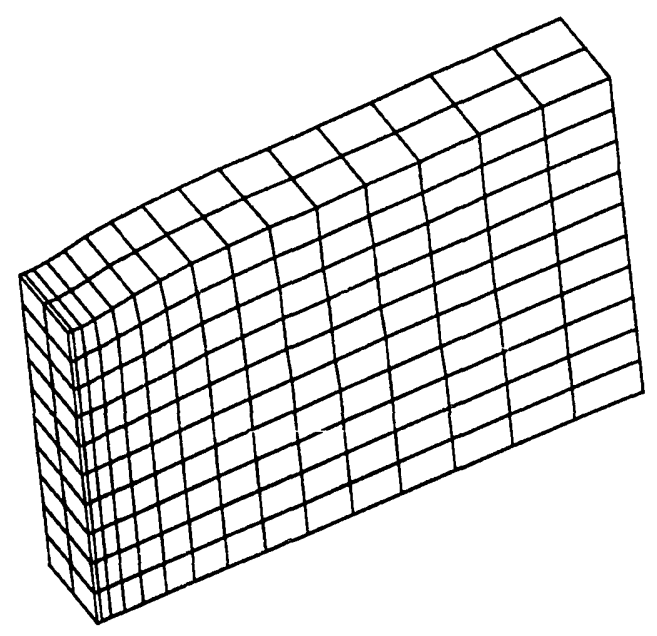

PUNCH TRAVEL $=0.15$ ins

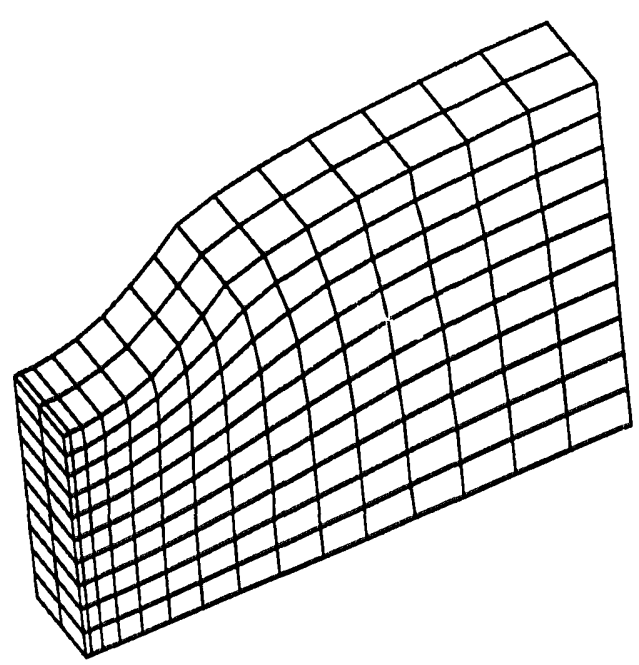

PUNCH TRAVEL $=0.75$ ins

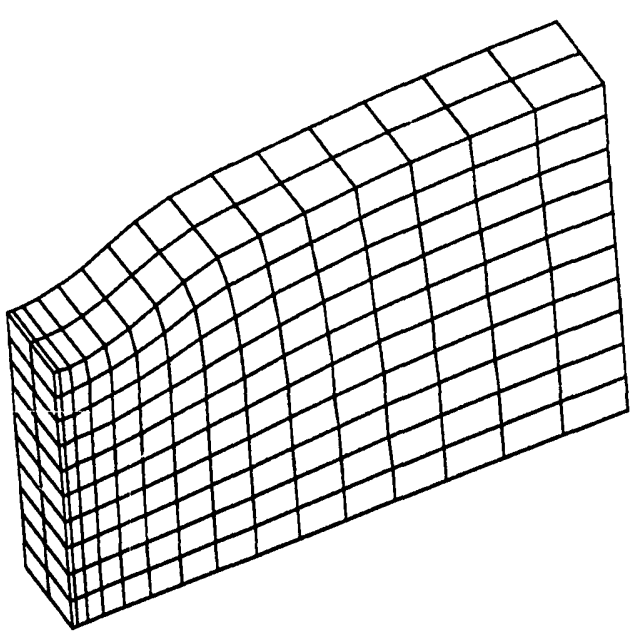

PUNCH TRAVEL $=0.45$ ins

Fig. 34. Deformation patterns at various stages in the indentation problem.

the vertical. The vertical force exerted by the punch on the slab was calculated from the pressure in the contact zone and this is shown for various stages in Fig. 37. Also Fig. 38 shows the variation of maximum contact pressure with the movement of the punch. Finally the sinking depth of the punch is plotted in terms of the vertical load in Fig. 39 and these show good agreement with [45].

EXAMPLE 6. Side extrusion problem. This is a problem of metal forming in which the Lagrangian description proves to be ineffective in boundary representation. In such forming 


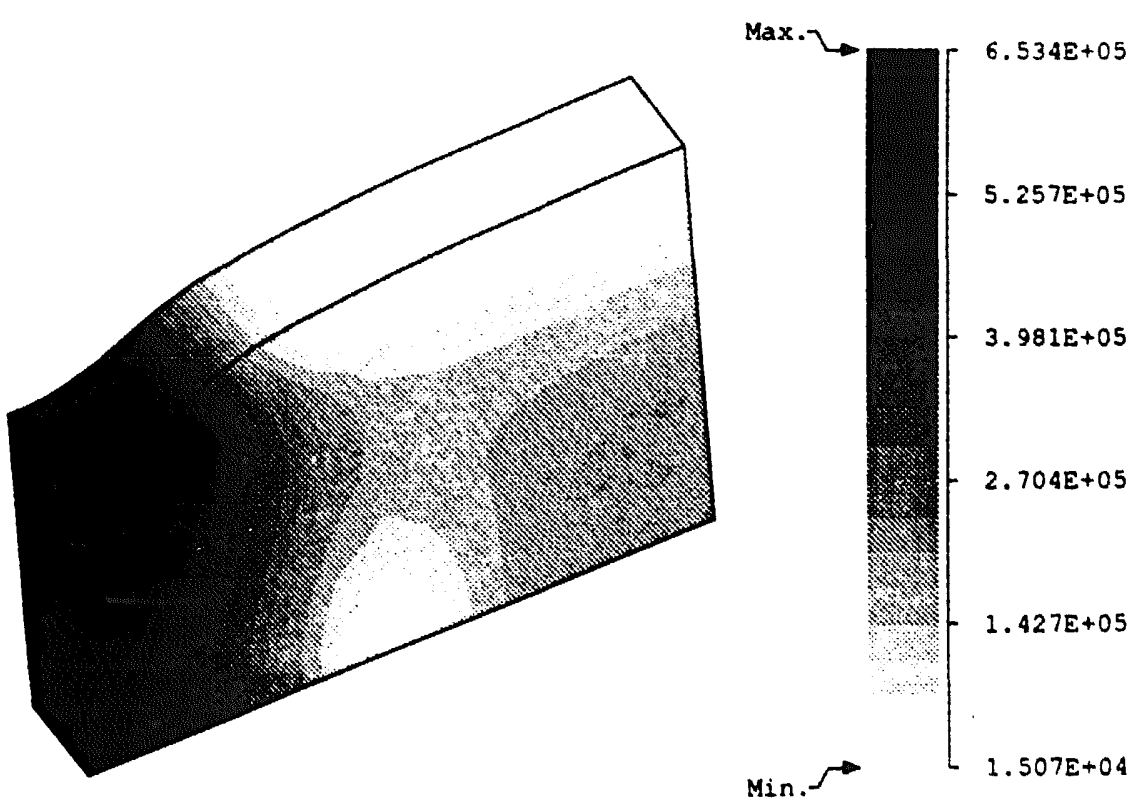

PUNCH TRAVEL $=0.45$ ins
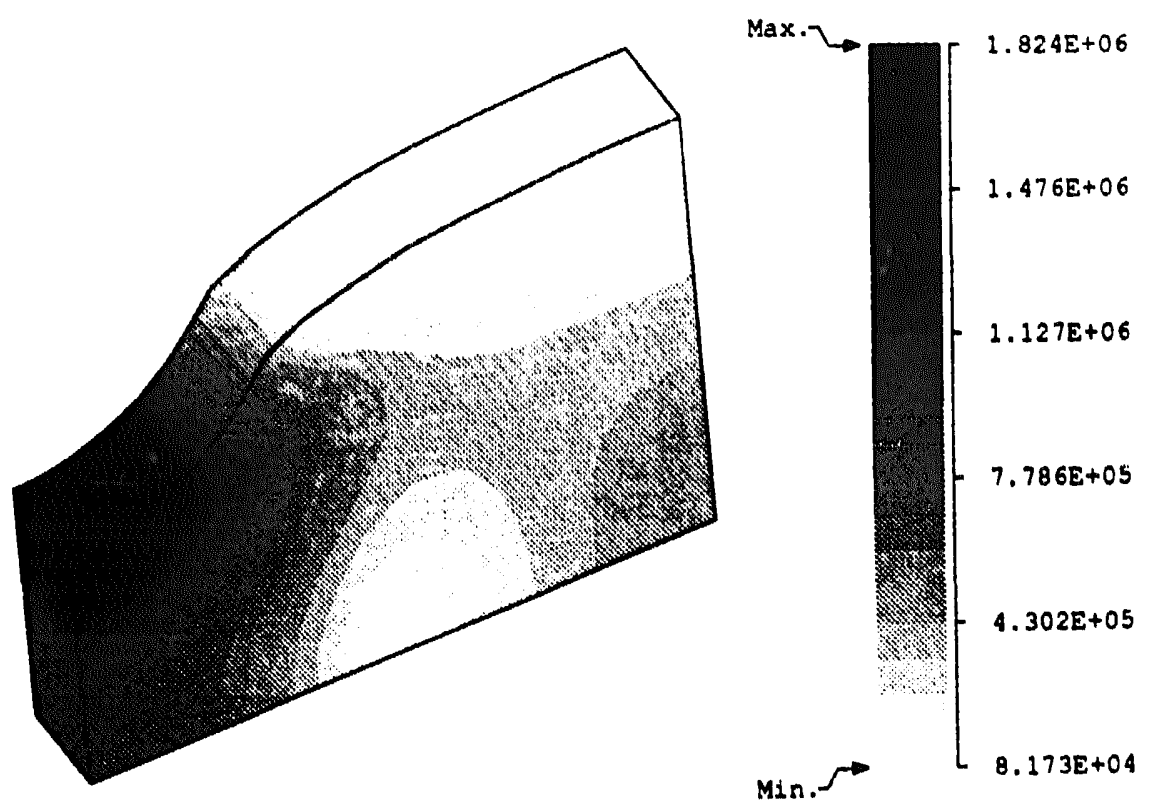

PUNCH TRAVEL $=0.99$ ins

Fig. 35. Equivalent stress contours (psi) in the indentation problem. 


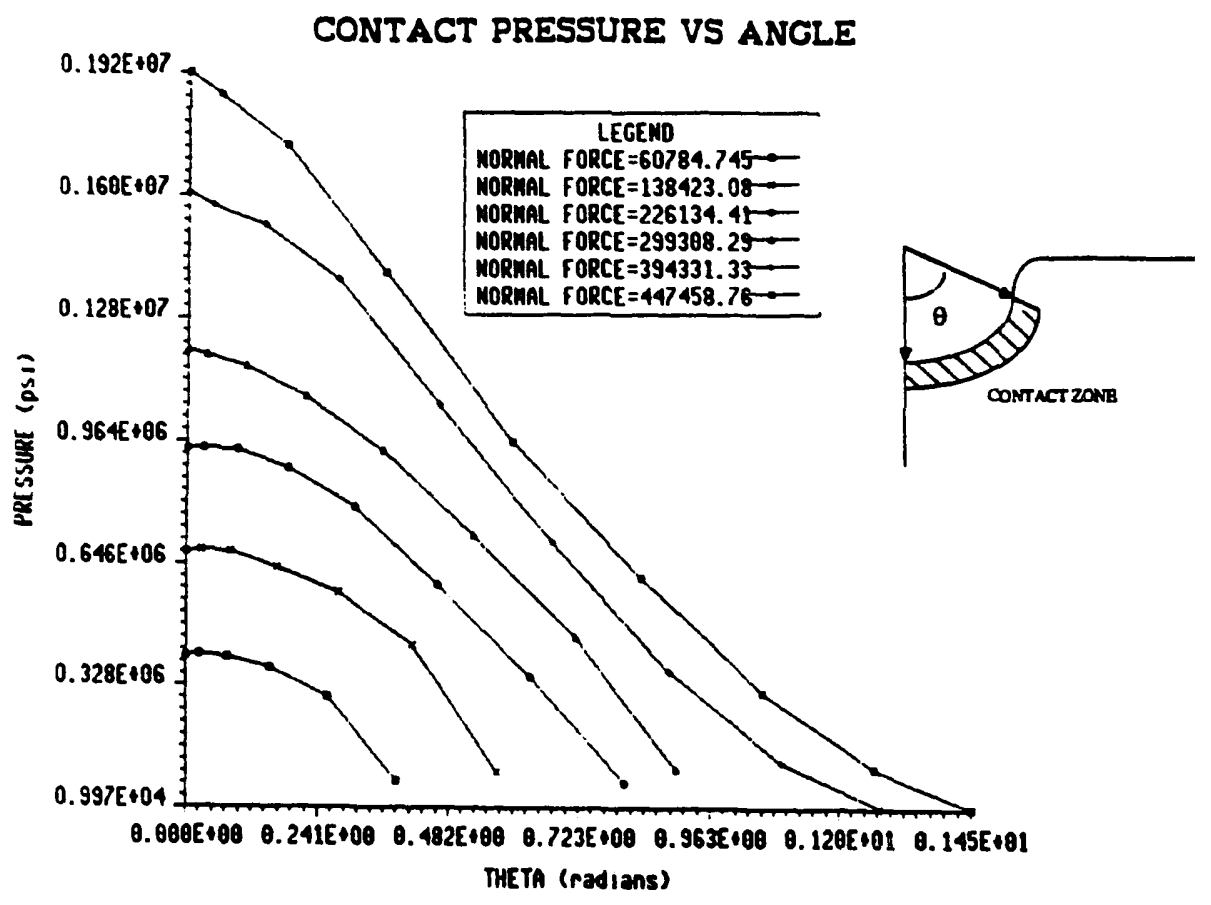

Fig. 36. Contact pressure (psi) as a function of contacting angles (radians).

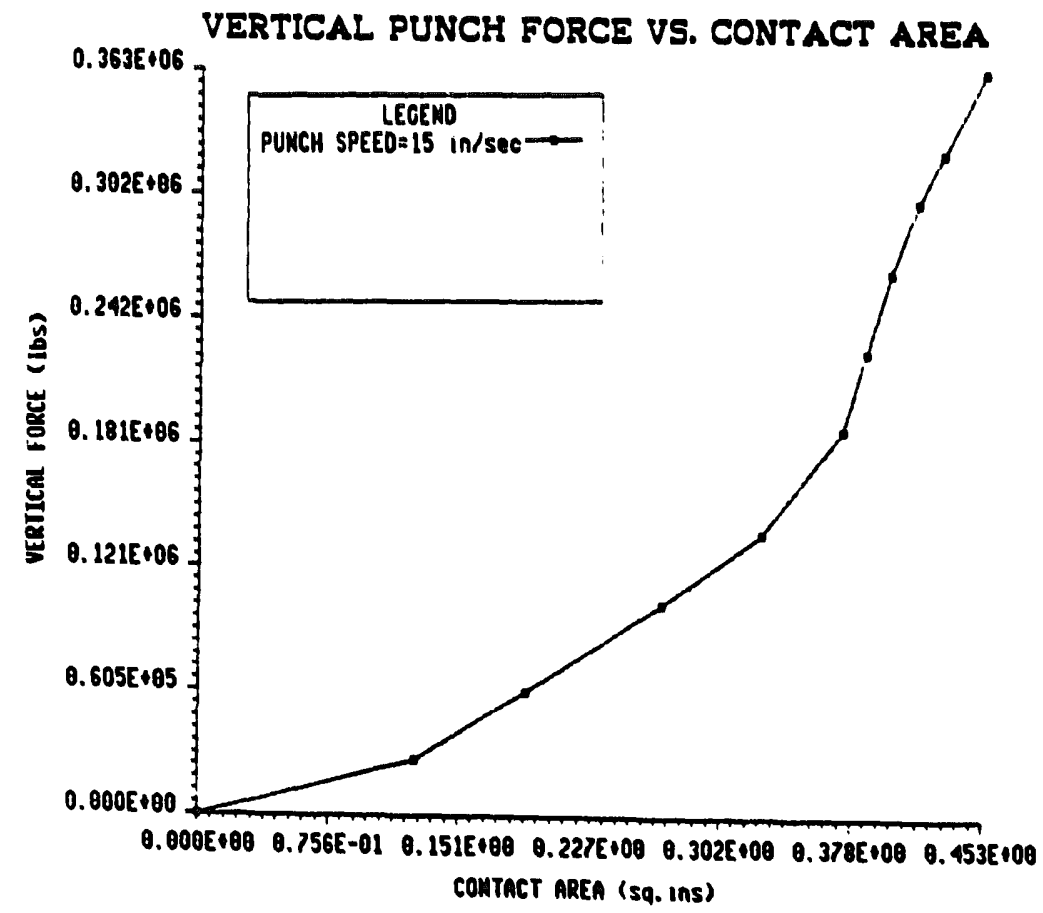

Fig. 37. Vertical punch force (lbs) as a function of contact area $\left(\mathrm{in}^{2}\right)$. 


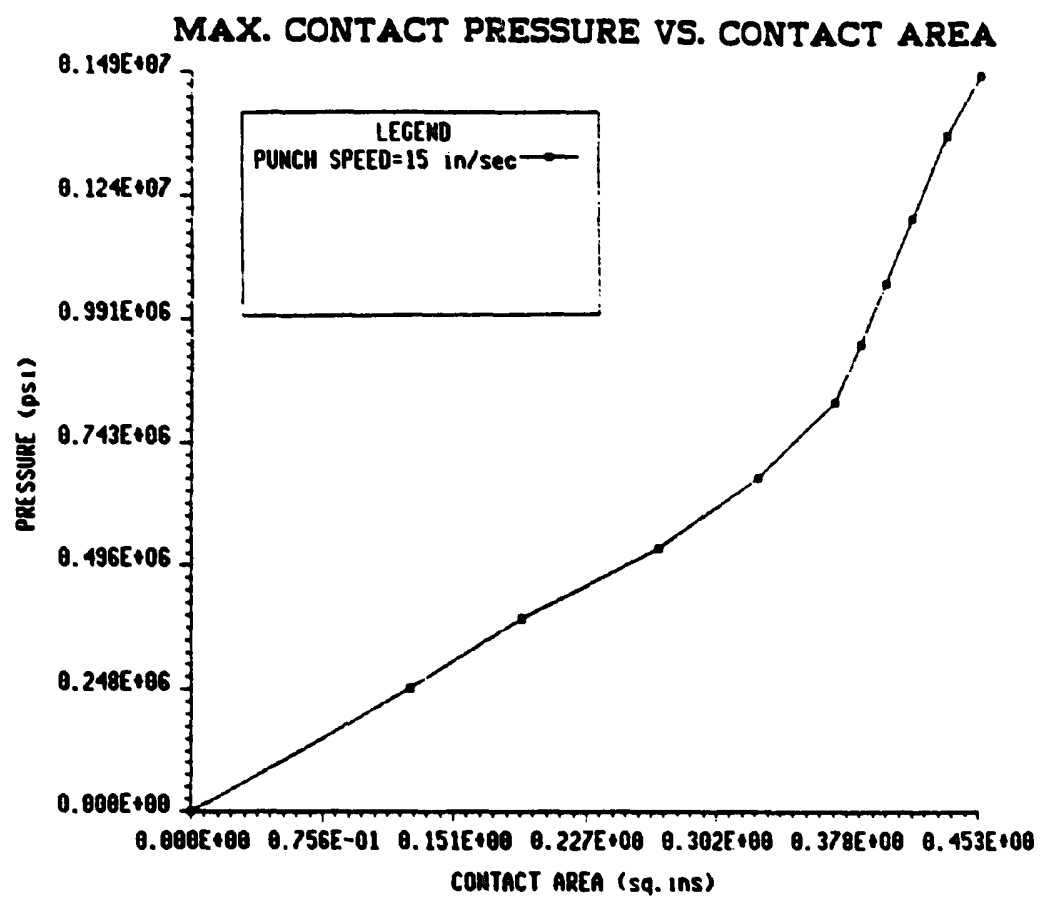

Fig. 38. Maximum contact pressure (psi) as a function of contact area $\left(\mathrm{in}^{2}\right)$.

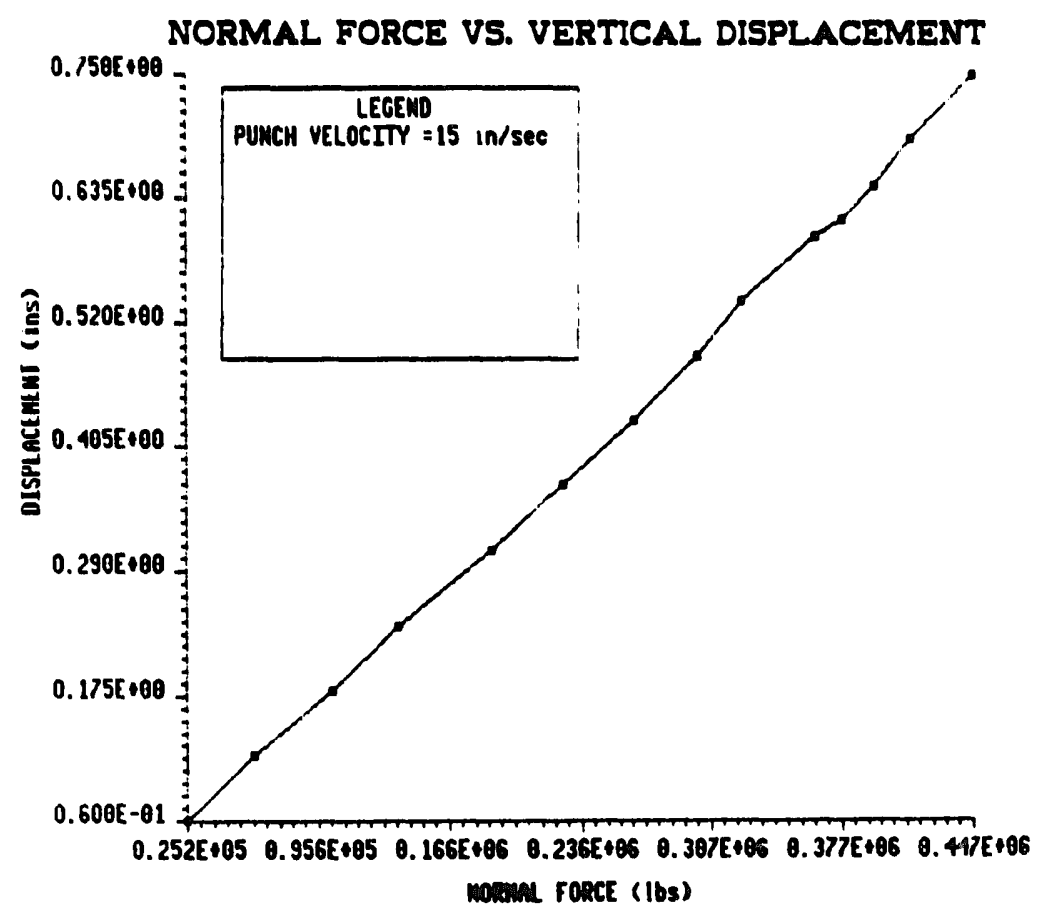

Fig. 39. Punch travel (in) as a function of vertical punch force (lbs). 
operations, material is extruded by a punch through a slit on the side of the die as is shown in Fig. 40. The traditional Lagrangian methods cannot represent the edge of the slit properly even with remeshing. Also the mesh associated with the material which is being extruded out of the slit becomes extremely distorted in this description. As a result, the use of the ALE description for this problem is almost indispensible in this analysis.

Only a half of the elastic-viscoplastic extrudate is modelled in this example from symmetry considerations. The contact between the surfaces of the die and punch and the material is assumed to be frictionless. The dimensions and material parameters are indicated in Fig. 40. The process is executed for $0.2 \mathrm{sec}$ corresponding to 40 percent reduction in the height of the extruded material and the computation is carried out in 20 equal steps. Evidently, the mesh moving scheme is one of the most demanding aspects of the ALE description in such a problem. Several different combinations of mesh descriptions and node movements were experimented with, in this example. It is apparent that in order to represent the slit precisely, nodes coinciding with the top of the slit should be made Eulerian. However, as shown in Fig. 41, certain difficulties are encountered with most of the schemes if we keep these nodes Eulerian at all times. For example, Fig. 41(a) shows the results obtained by an entirely Eulerian mesh with Lagrangian nodes, at the opening. The analysis stopped after 12 steps corresponding to a reduction of 24 percent, because the elements outside of the die became

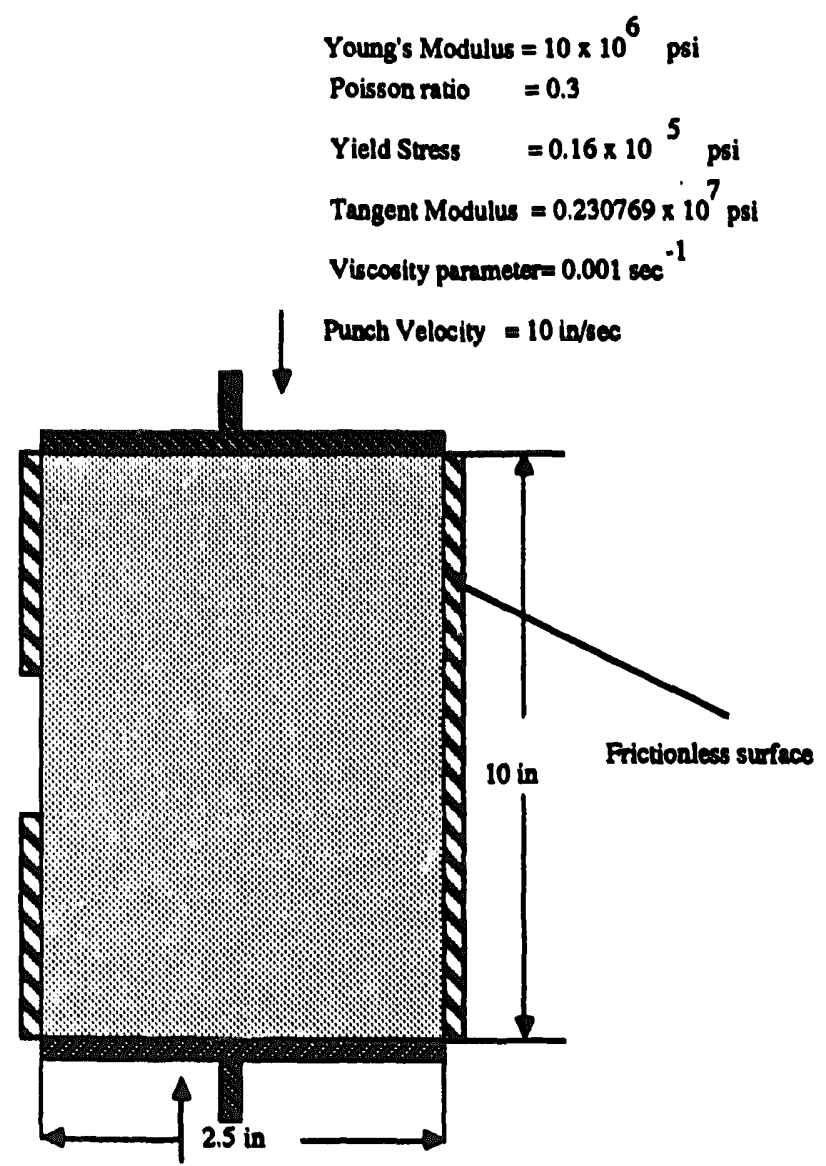

Fig. 40. Section view of a solid material in a die being side extruded by a rigid punch. 


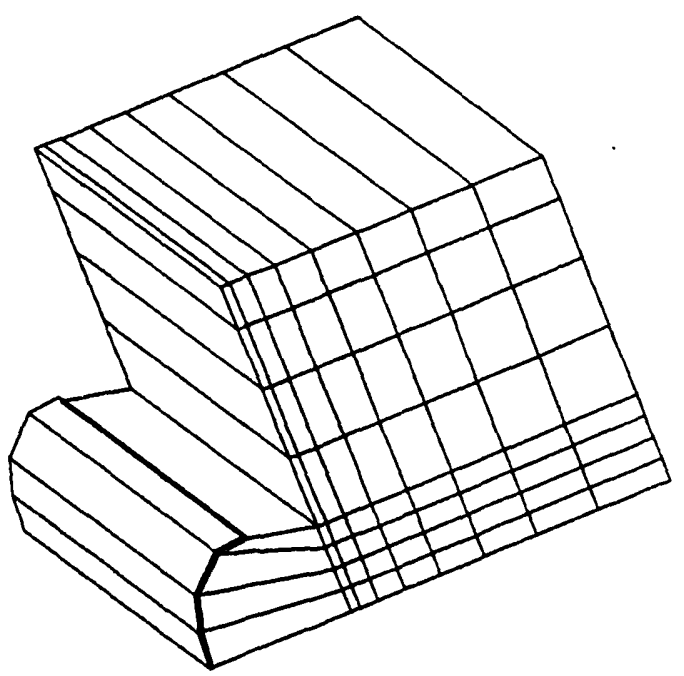

STEP NR. 12

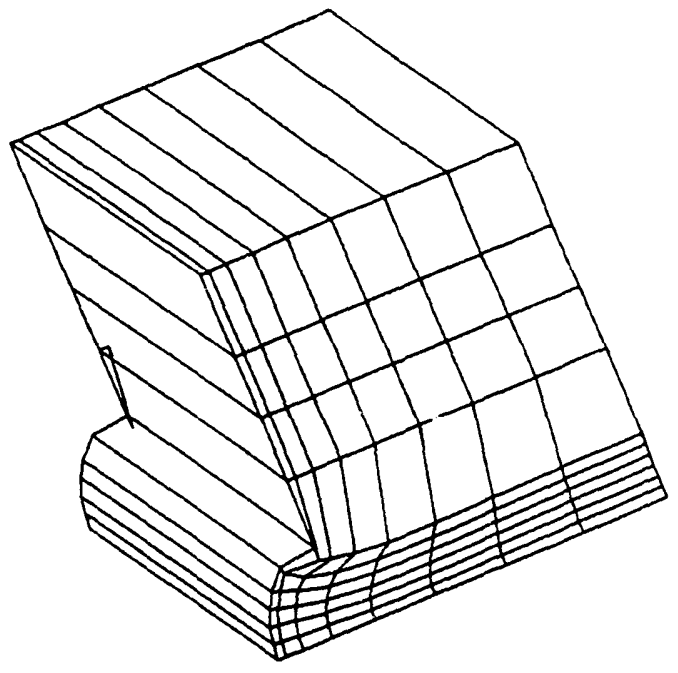

STEP NR. 7

Fig. 41. Some unsuccessful mesh moving schemes.

very long. This resulted in difficulties with proper interpolation for the material path and hence unreasonable material displacements were obtained. A second description, shown in Fig. 41(b) represents a mesh in which the nodes below the level of the top edge of slit are made Lagrangian. Clearly, the elements become entangled after a height reduction of 14 percent. This prompted the use of partial Eulerian description of the nodes in contact with the edge of the opening. Here, a set of nodes are held Eulerian at the edge till 20 percent reduction (10 steps) is achieved, after which they become Lagrangian and move with their respective material points. Meanwhile the row above these are ALE nodes, which are made to move down in such a way that they replace the former set of nodes from the 11th step. This can be seen from Fig. 42. The nodes at the lower portion within the opening are made Lagrangian to portray the material movement and also for computational efficiency. Obviously this necessitates the introduction of transient modes to smoothen the behavioral difference between the Lagrangian and Eulerian nodes. A weighted averaging scheme given by

$$
W_{l}^{n+1}=\frac{1}{N} \sum_{J} w_{j} W_{J}^{n}
$$

is used to assign nodal velocities in this region. Here $w_{J}$ represents a weighting function associated with a $J$-th node that surrounds the $I$-th node, $N$ is the total number of nodes around the $I$-th node and $n$ corresponds to the time step. The success of this description can be easily inferred from Fig. 42 in which the analysis was successfully carried out till $40 \%$ reduction and further. The corresponding motion of the material points and the equivalent 


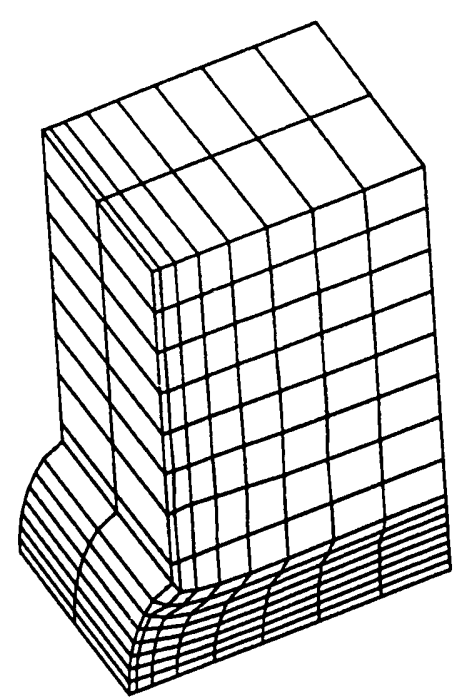

$12 \%$ REDUCTION

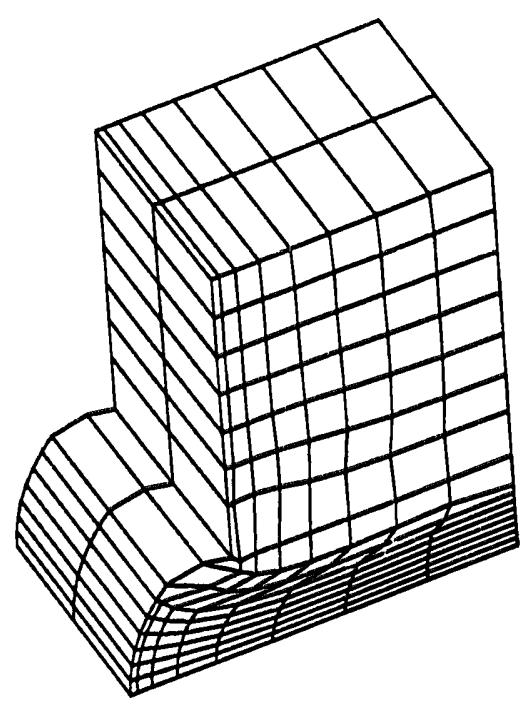

$22 \%$ REDUCTION

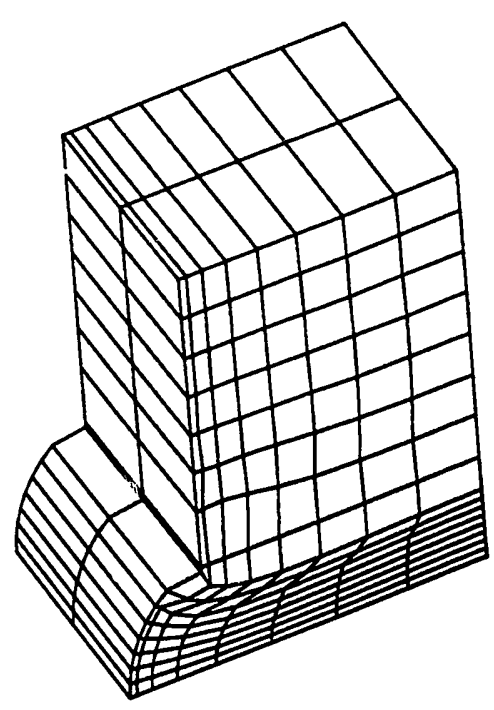

$18 \%$ REDUCTION

Fig. 42. Mesh movements in a side extrusion problem.

stress contours are shown in Figs. 43 and 44. The sudden opening in the wall of the die introduces some instabilities which are visible in Fig. 43.

EXAMPLE 7. Back extrusion problem. As a final example, we demonstrate the ability of the ALE description to alleviate the typical problems encountered in the simulation of a back extrusion process by the Lagrangian formulation. The description of the problem is illustrated in Fig. 45, where a rigid prismatic punch of transverse thickness 1.5 inches is back extruding 


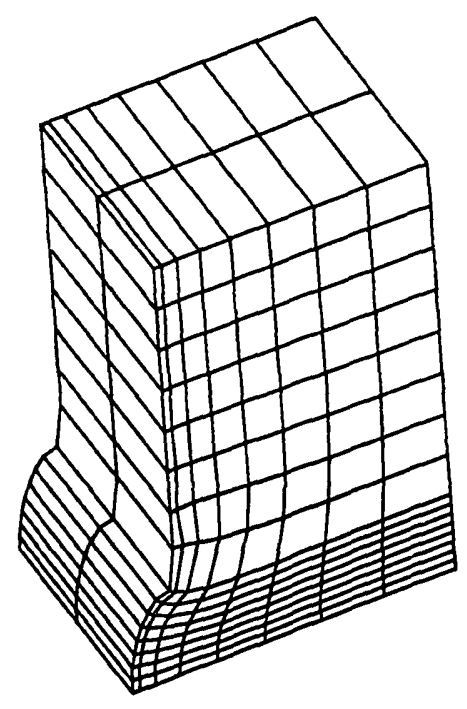

$12 \%$ REDUCTION

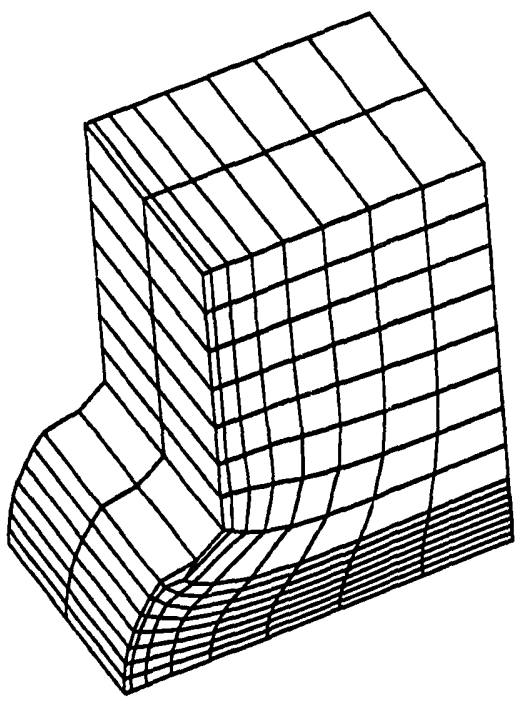

$22 \%$ REDUCTION

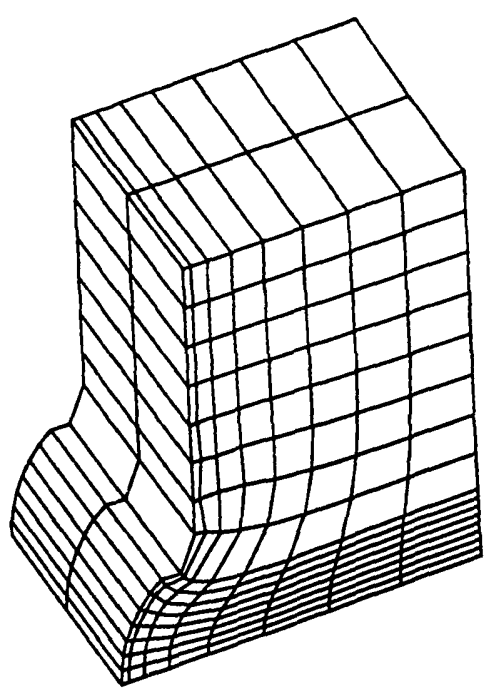

$18 \%$ REDUCTION

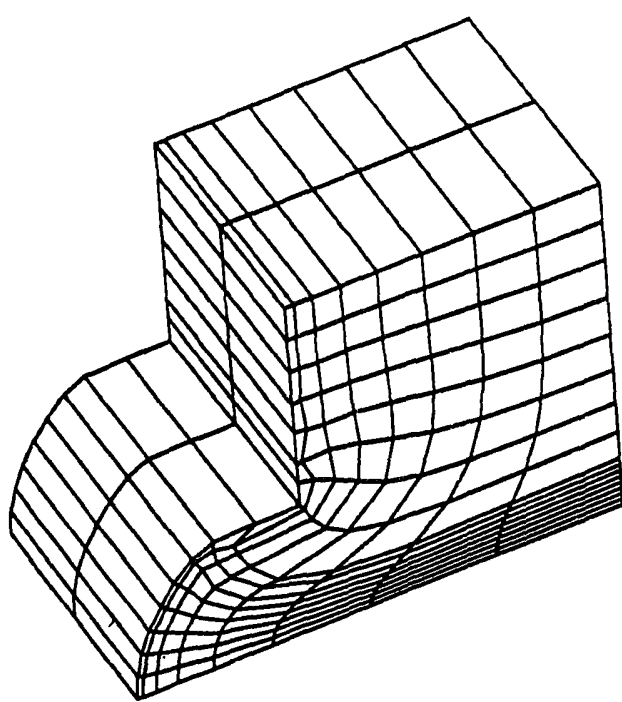

$40 \%$ REDUCTION

Fig. 43. Material movements in a side extrusion problem.

an elastic-viscoplastic billet of the same thickness. The material parameters are indicated in Fig. 45. An assumption that all contact surfaces are frictionless is made. It is intended for the punch to cause a $40 \%$ reduction in the height of the billet under the flat punch surface with successive strokes. The process continues for an interval of $0.2 \mathrm{sec}$ which is temporally discretized into 20 equal steps for analysis. 


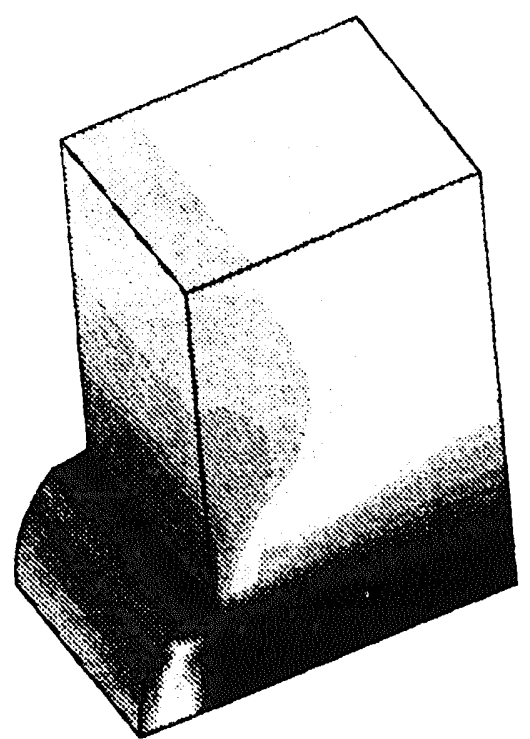

$18 \%$ REDUCTION

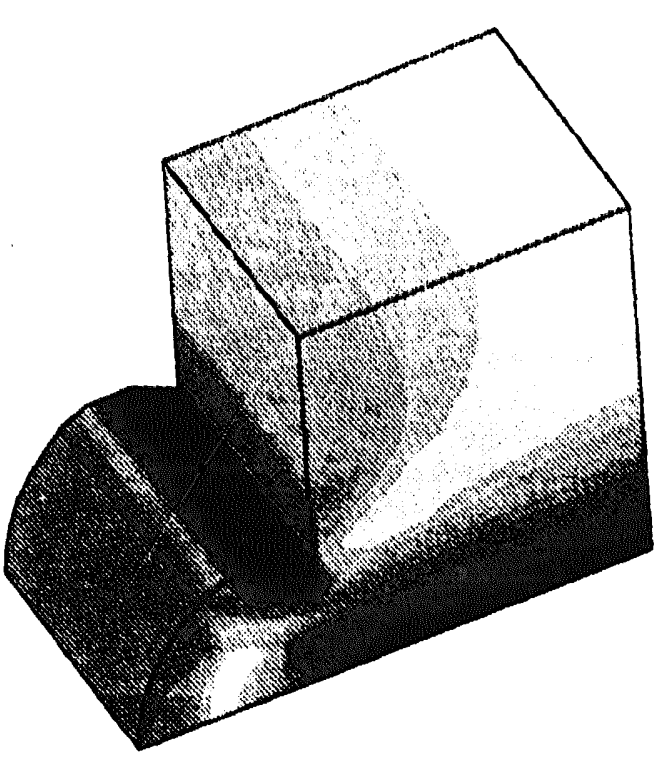

4) \% REDUCTION
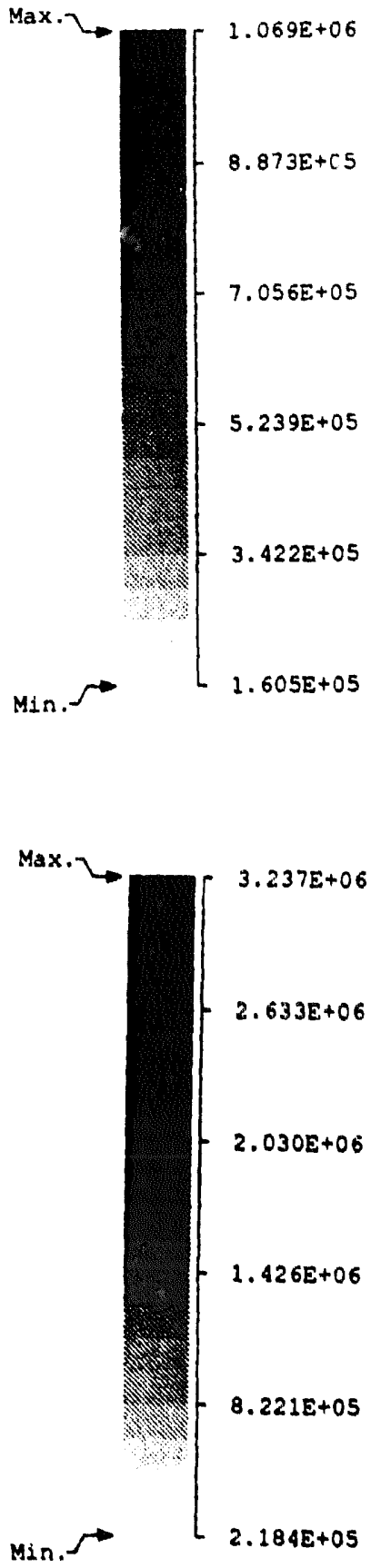

Fig. 44. Equivalent stress (psi) contours in the side extrusion problem. 


\begin{tabular}{|c|c|c|}
\hline Young's Modulus & $=10 \times 10^{6}$ & psi \\
\hline Poisson ratio & $=0.3$ & \\
\hline Yield Stress & $=16 \times 10^{3}$ & \\
\hline $\begin{array}{l}\text { Tangent Modul } \\
\text { Viscosity paran }\end{array}$ & $\begin{array}{l}s=0.230769 \\
\text { eter }=0.001 s\end{array}$ & $\begin{array}{l}x_{-1}^{7} \mathrm{psi} \\
\mathrm{sec}\end{array}$ \\
\hline Punch Velocity & $=10 \mathrm{in} / \mathrm{sec}$ & \\
\hline
\end{tabular}

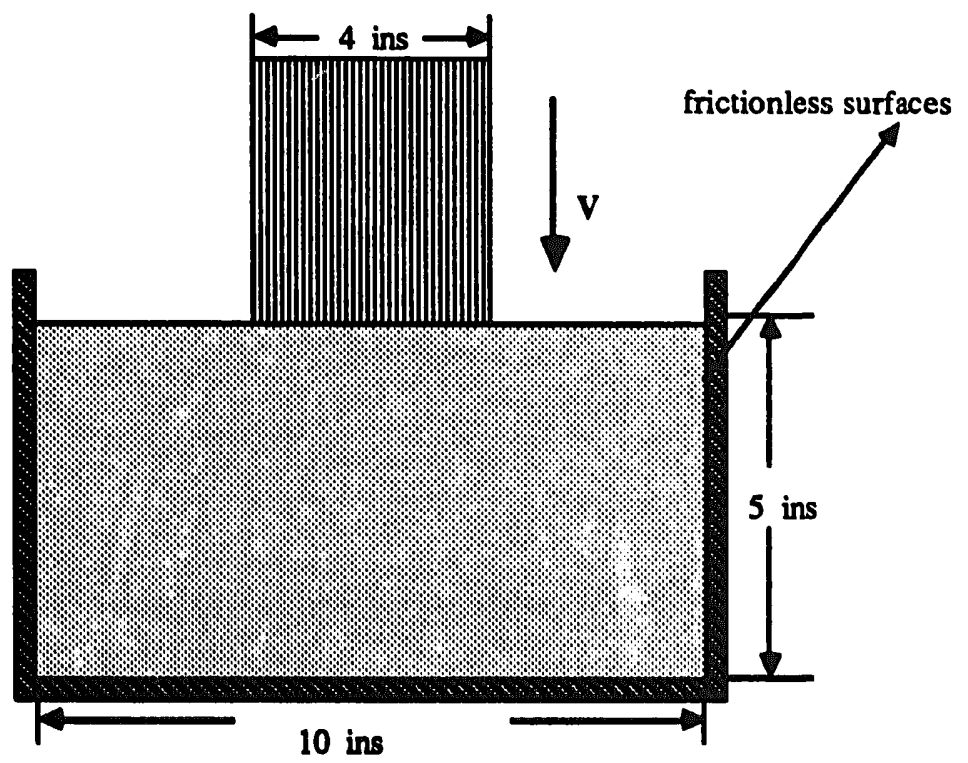

Fig. 45. Section view of the back extrusion problem.

Similar problems with the Lagrangian formulation have been solved by Cheng and Kikuchi [1] and Gelten and Konter [46] using mesh rezoning techniques for rate-independent plasticity. However, it is beneficial to use the ALE description to solve this problem with adequate accuracy and efficiency. Because of the problem symmetry, only half of the workpiece is analyzed. To start with, we model the billet with a majority of Lagrangian nodes with the exception of nodes coinciding with the edge of the punch, which are glued to the punch. The sharp corner of the punch causes the material around it to deform excessively and as a result the elements in the vicinity are severely distorted. This can be seen from Figs. 46 and 47. The interaction between neighboring Lagrangian and ALE nodes causes element entangling, thus stopping the analysis. The blow up of the distorted zone in Fig. 47 is just before this entanglement occurs at $20 \%$ height reduction.

A few schemes for effectively moving the nodes around the sharp edge of the extruding punch were experimented with. First, a portion of the mesh which is vulnerable to excessive distortion is automatically constructed in each step by elliptic mesh generation techniques after 


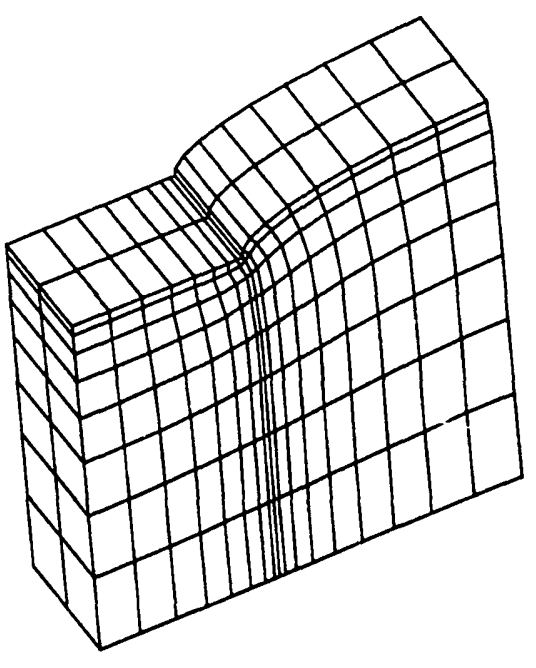

$10 \%$ REDUCTION

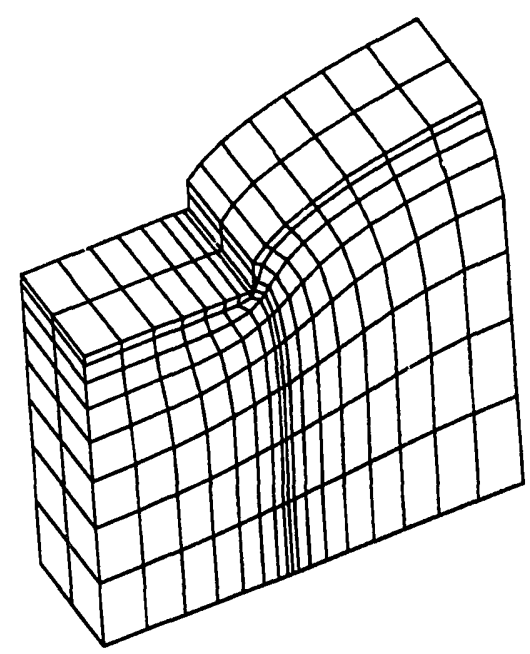

$18 \%$ REDUCTION

Fig. 46. Element crashing in a nearly Lagrangian mesh.

$18 \%$ height reduction. In this method, variational techniques may be applied to minimize a linear combination of a measure of grid smoothness, orthogonality and volume variation resulting in a set of elliptic partial differential systems, e.g. the Laplace equation or the more general Poisson equation

$$
\nabla^{2} \xi^{i}=P^{i}, \quad i=1,2,3,
$$

where $\xi^{i}$ refer to the curvilinear coordinate system of the physical domain and $P^{i}$ are the control functions which may be implemented to control the spacing and orientation of coordinate lines. The deformed configurations of the billet demonstrated in Fig. 48 are results of this scheme. The control function $\boldsymbol{P}$ was adjusted to yield favorable meshes. A typical partially elliptically generated mesh at an intermediate step may be observed from Fig. 49(a).

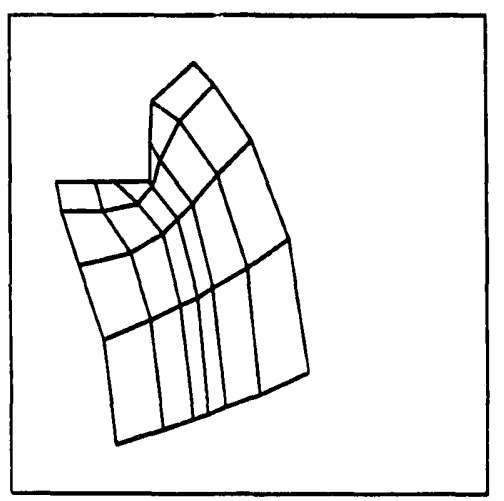

Fig. 47. Blow up of the section of element crashing in a nearly Lagrangian mesh. 


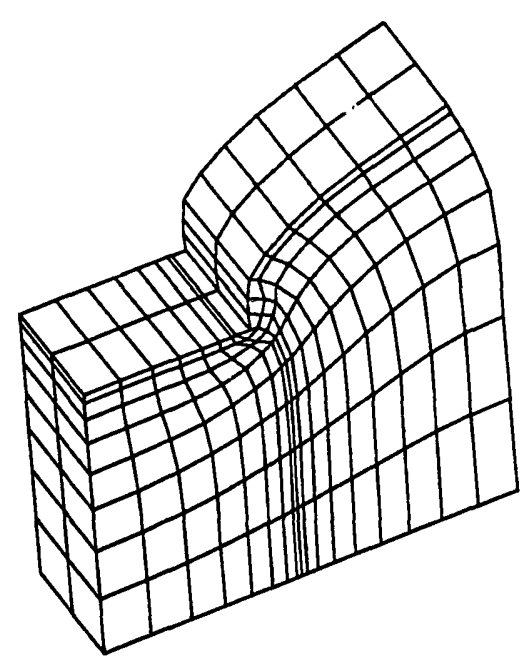

$26 \%$ REDUCTION

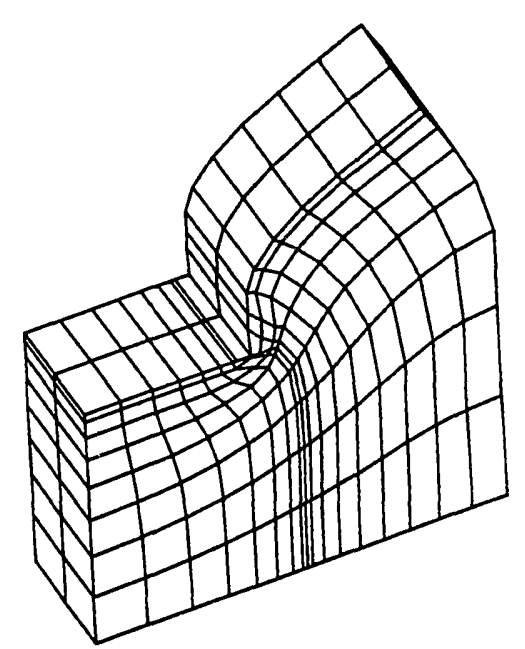

$34 \%$ REDUCTION

Fig. 48. Deformation patterns with the mesh by elliptic generator.

However, as the contacts with the vertical edges of the punch increase, the boundary of the zone for elliptic generator admits a sharp corner and this results in the excessive distortion of the mesh as seen in Fig. 49(b). This caused the process to stop after 34\% deformation under the punch.

Alternately, the algebraic mesh generator, which involves transformation into a computational domain to compute the interior points of the domain was tried. As in the previous case, this generator was activated every alternate step after $18 \%$ height reduction. A section view of the consequently generated mesh is seen in Fig. 53. The process was successfully executed for

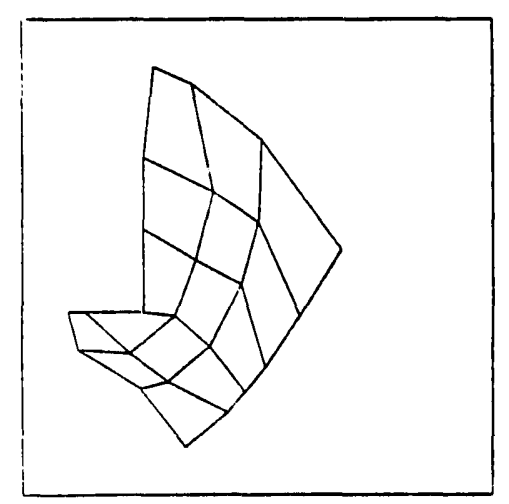

(a)

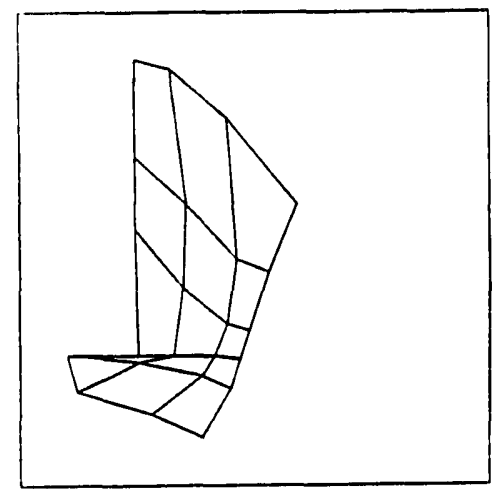

(b)

Fig. 49. Blow up of the sections of elliptically generated mesh (a) at an intermediate step and (b) when the elements become overdistorted. 


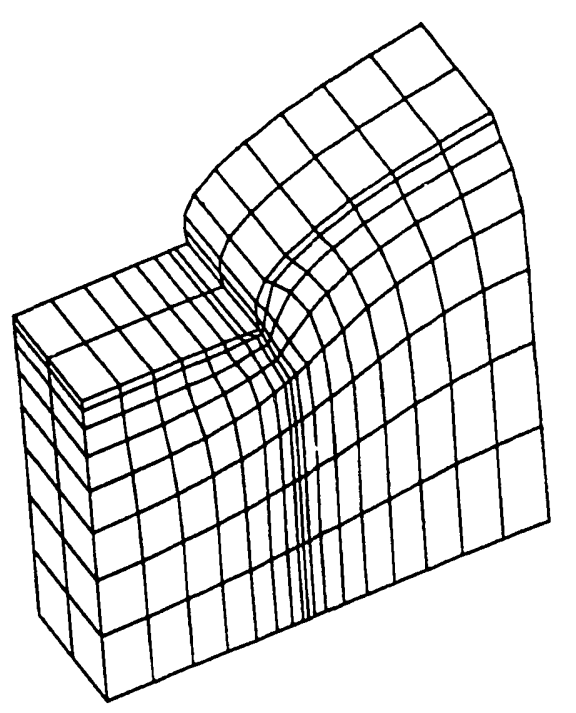

$20 \%$ REDUCTION

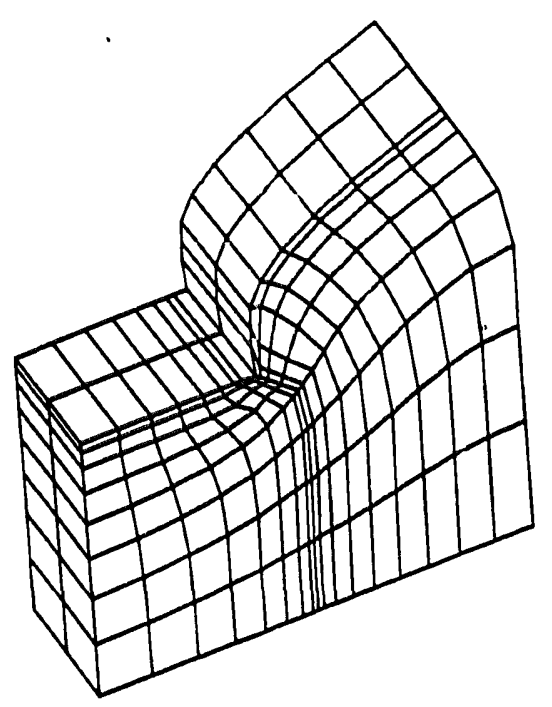

$32 \%$ REDUCTION

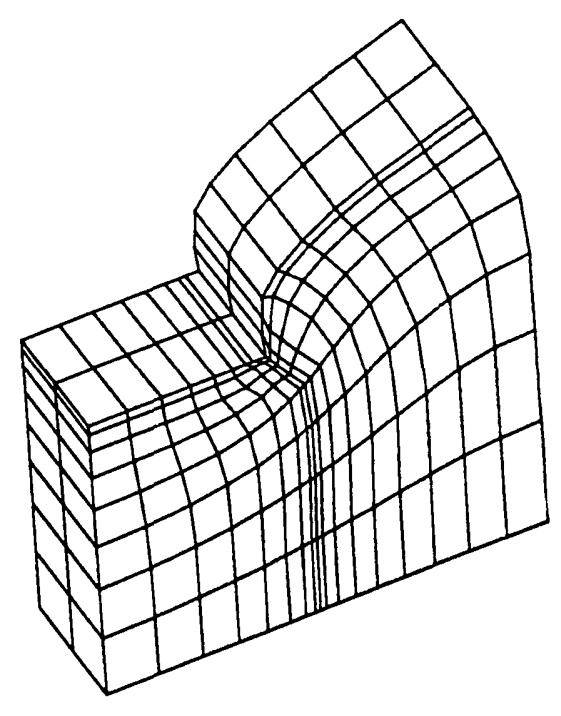

$28 \%$ REDUCTION

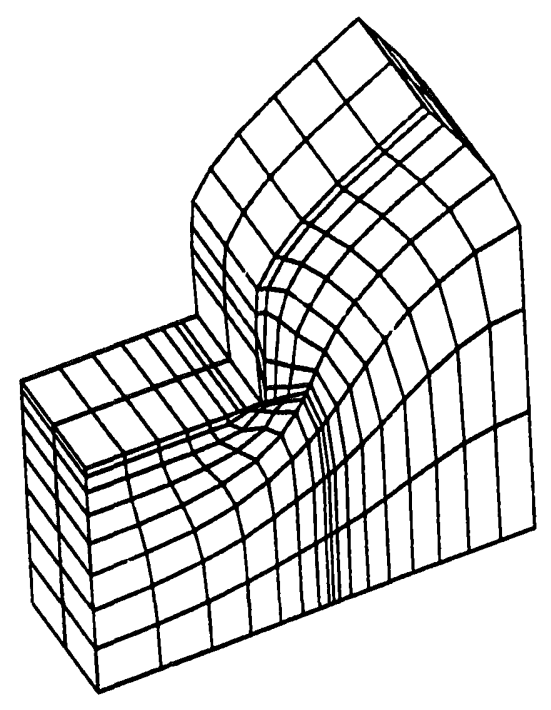

$40 \%$ REDUCTION

Fig. 50. Deformation patterns with contact edge zone generated by algebraic mesh generator.

$40 \%$ deformation with this scheme. Figure 50 clearly exhibits the FEM mesh at various stages of the process. The corresponding material flow is given in Fig. 51, while Fig. 52 illustrates the equivalent stress contours for some representative steps. The applied load under the punch is computed from the pressure distribution and is plotted with the punch movement in Fig. 54, while Fig. 55 shows the pressure distribution. 

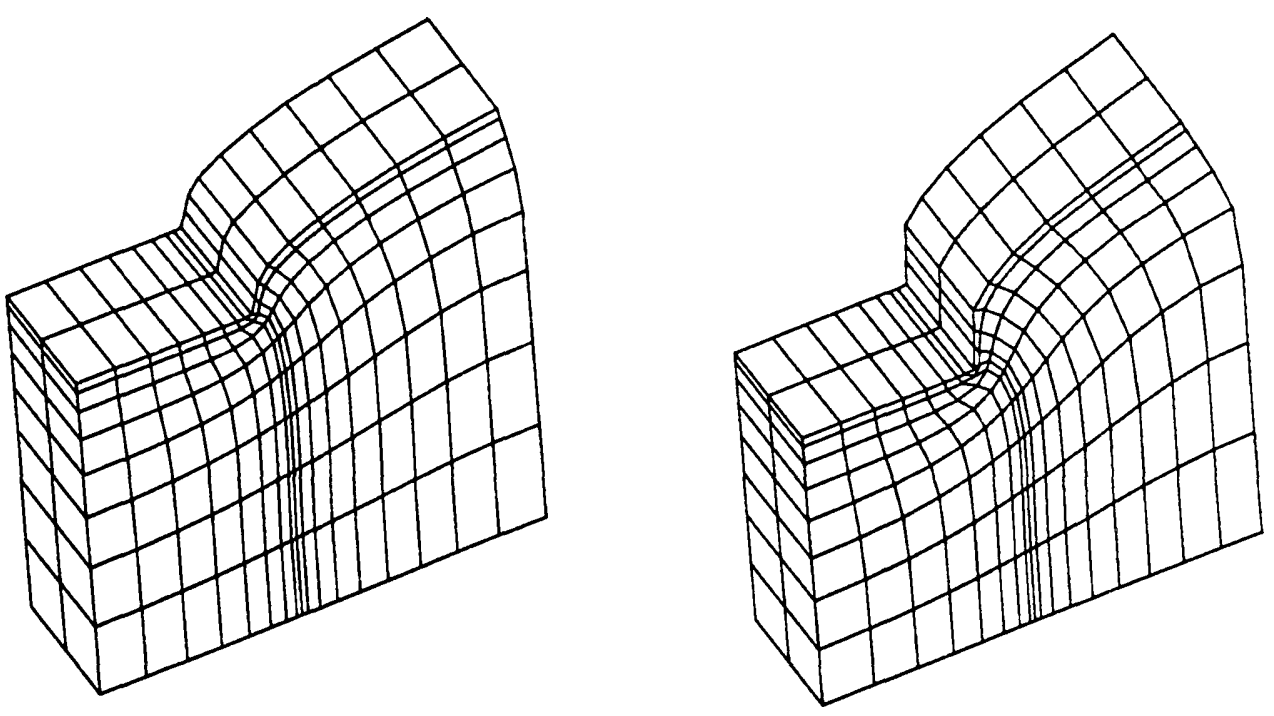

$20 \%$ REDUCTION

$28 \%$ REDUCTION

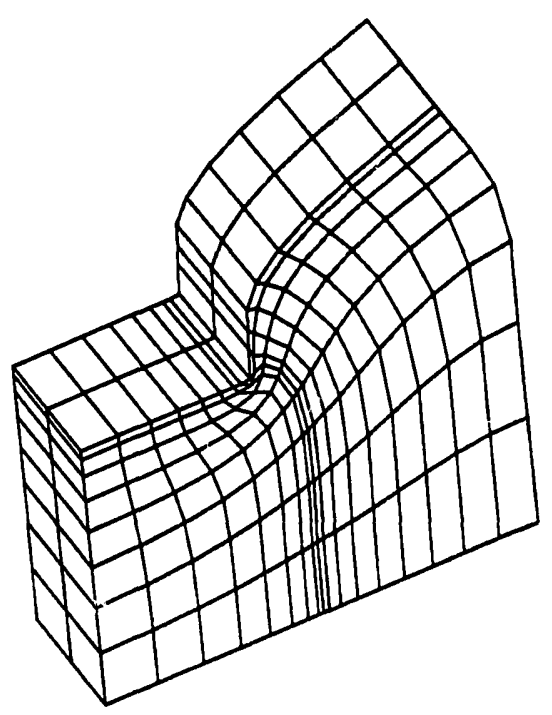

$32 \%$ REDUCTION

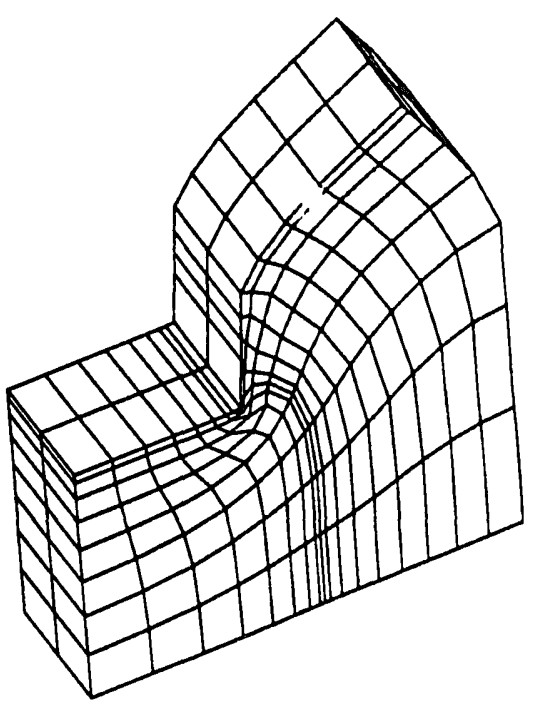

$40 \%$ REDUCTION

Fig. 51. Material movement in the back extrusion problem. 

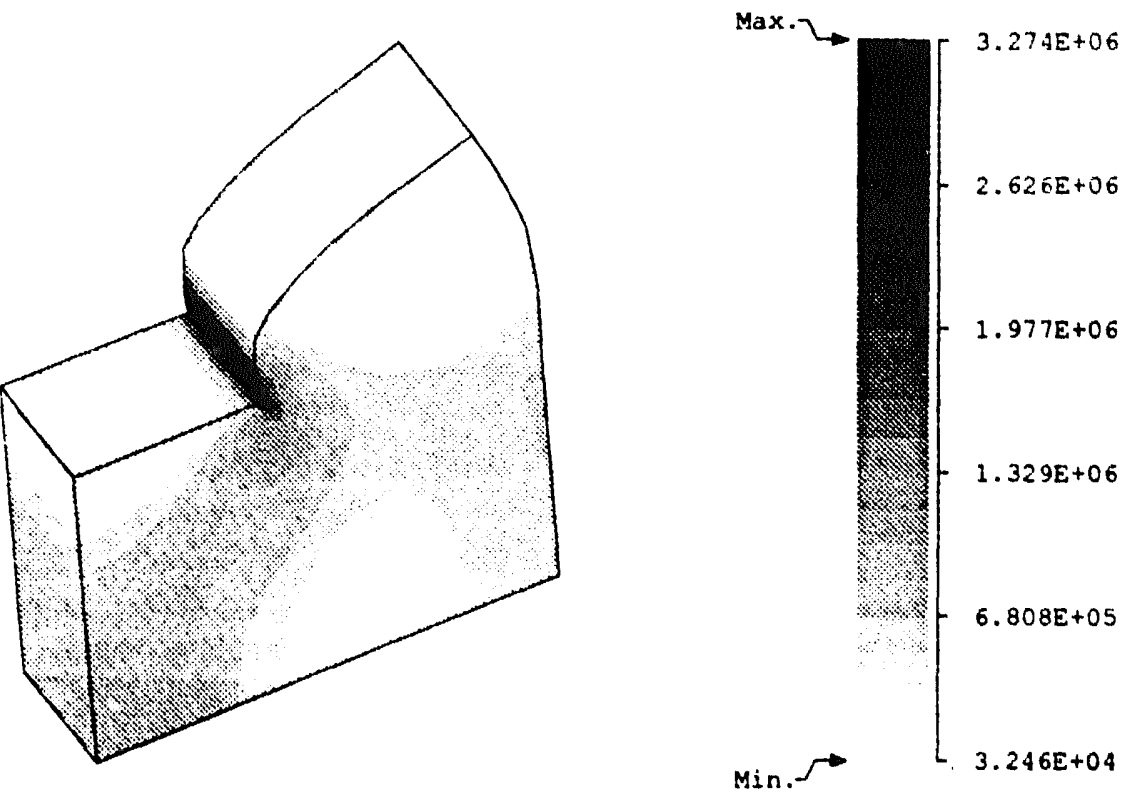

$28 \%$ REDUCTION
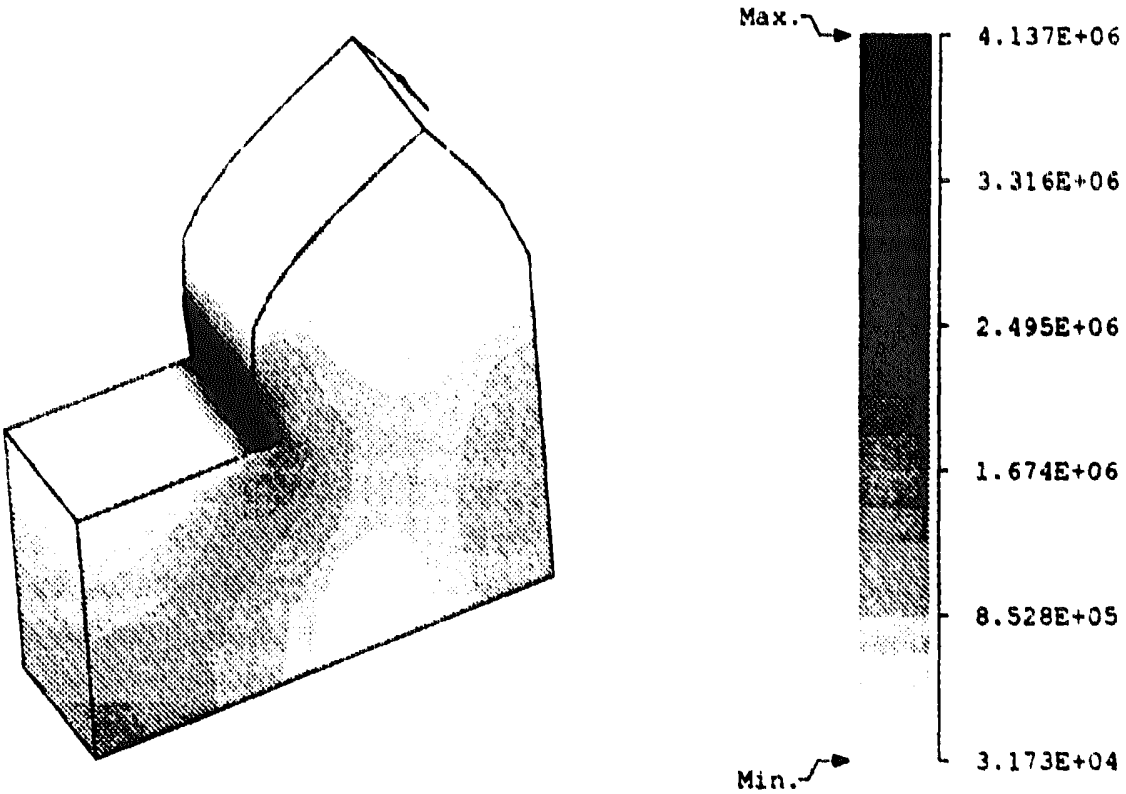

$40 \%$ REDUCTION

Fig. 52. Equivalent stress contours (psi) in the back extrusion problem. 


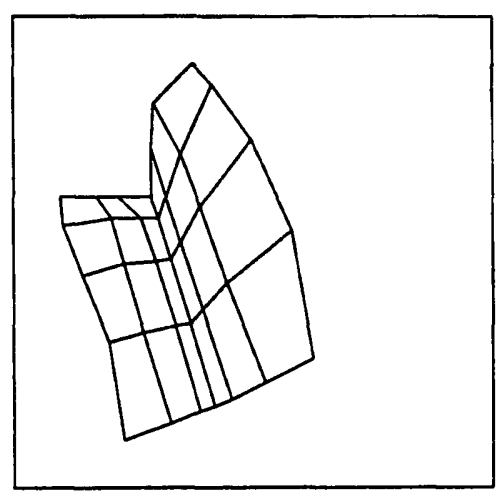

Fig. 53. Blow up of the section of algebraically generated mesh at a time step.

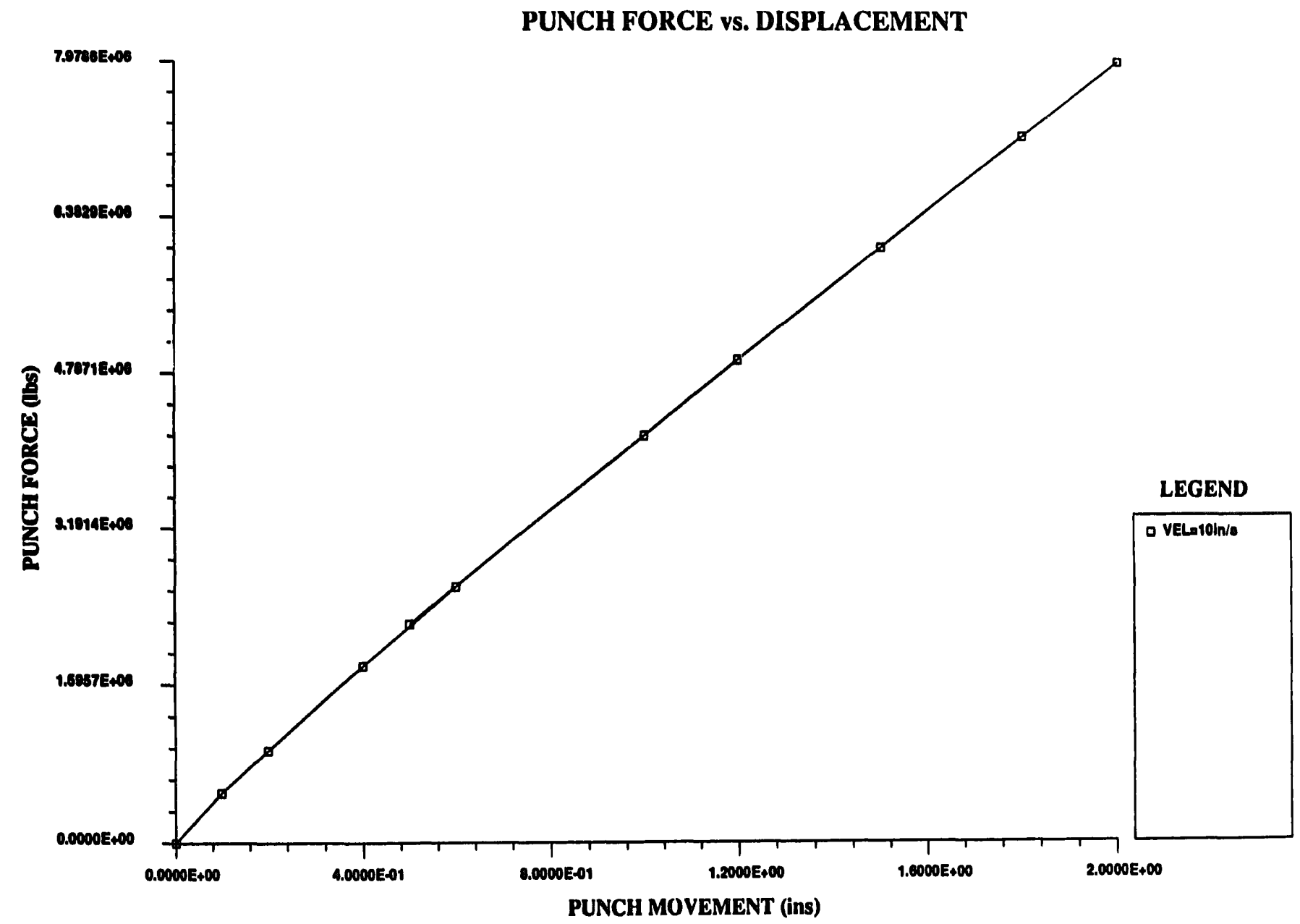

Fig. 54. Total force (lbs) exerted by the punch with respect to the punch movement (in). 


\section{CONTACT PRESSURE UNDER PUNCH}

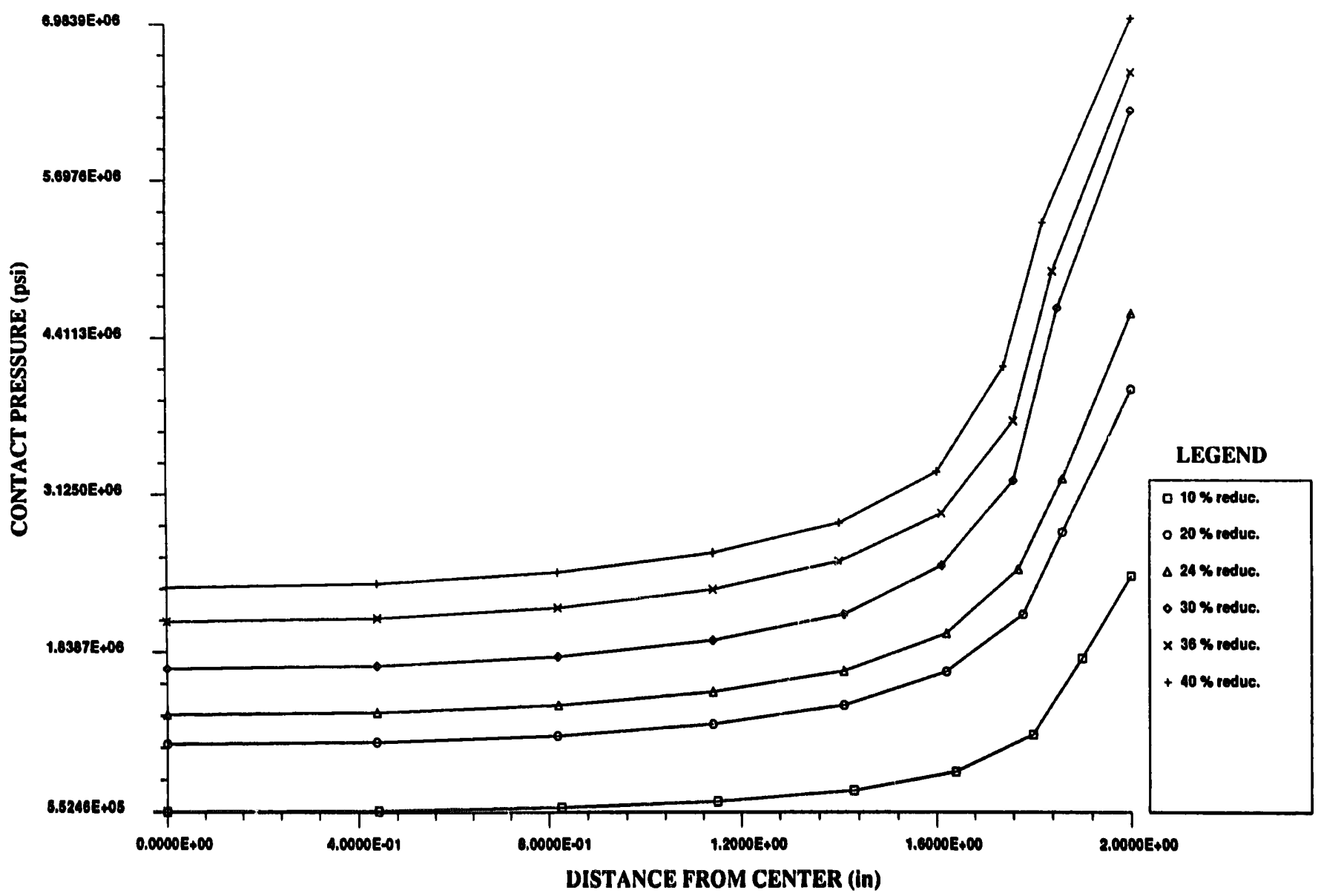

Fig. 55. Contact pressure (psi) under the punch at different stages.

\section{Conclusion}

This paper elucidates the power of the arbitrary Lagrangian Eulerian finite element method to solve various large deformation problems of elastic-viscoplastic materials, especially with complex boundary descriptions. The algorithms described in this paper may find direct application in metal forming analysis and fracture mechanics problems where many compromises are often made in accordance with the limitations of the conventional methods. The overall algorithm proved to be numerically stable and reasonably accurate in the sense that the results obtained are in very good accordance with physical experience. Temperature effects and more efficient adaptive mesh moving schemes based on error analysis have not been considered in this paper and will be addressed in a forthcoming paper.

\section{Acknowledgment}

The authors are grateful to Professor M. Ortiz for his valuable comments on the constitutive modeling and linearization techniques used in this paper. They would also like to 
acknowledge Dr. A. Kushner of ONR for his support of this research through ONR grant No. 0001485 k 0799.

\section{References}

[1] J.H. Cheng and N. Kikuchi, A mesh rezoning technique for finite element simulations of metal forming processes, Internat. J. Numer. Methods Engrg. 23 (1986) 219-228.

[2] C.W. Hirt, A.A. Amsden and J.L. Cook, An arbitrary Lagrangian-Eulerian computing method for all flow speed, J. Comput. Phys. 14 (1974) 227-253.

[3] J. Donea, P. Fasoli-Stella and S. Giuliani, Lagrangian and Eulerian finite element techniques for transient fluid-structure interaction problems. Trans. 4th Internat. Conf. on SMIRT, Vol. B (1977) 1-12.

[4] J.M. Kennedy and T.B. Belytschko, Theory and application of a finite element method for arbitrary Lagrangian-Eulerian and solids, Nucl. Engrg. Des. 68 (1981) 129-146.

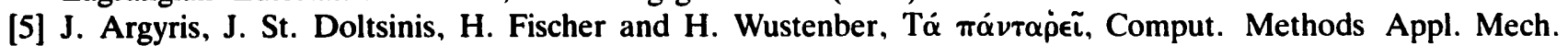
Engrg 51 (1985) 289-362.

[6] R.B. Haber, A mixed Eulerian-Lagrangian displacement model for large-deformation analysis in solid mechanics, Comput. Methods Appl. Mech. Engrg. 43 (1984) 277-292.

[7] D.J. Benson, An efficient, accurate, simple ALE method for nonlinear finite element programs, Comput. Methods Appl. Mech. Engrg. 72 (1989) 305-350.

[8] W.K. Liu, T. Belytschko and H. Chang, An arbitrary Lagrangian-Eulerian finite element method for path-dependent materials, Comput. Methods Appl. Mech. Engrg. 58 (1986) 227-245.

[9] T. Belytschko, An overview of semidiscretization and time integration procedures, in: T. Belytschko and T.J.R. Hughes, eds., Computational Methods for Transient Analysis, Vol. 1 (North-Holland, Amsterdam, 1983).

[10] I. Cormeau, Numerical stability in quasistatic elasto/viscoplasticity, Internat. J. Numer. Methods Engrg. 9 (1975) 109-127.

[11] V. Kumar, M. Morjaria and S. Mukherjee, Numerical integration of some stiff constitutive models of inelastic deformation, J. Engrg. Math. Tech. 102 (1980) 92-96.

[12] T.J.R. Hughes and R.L. Taylor, Unconditionally stable algorithms for quasi-static elasto/viscoplastic finite element analysis, Comput. \& Structures 8 (1978) 169-173.

[13] J.H. Argyris, L.E. Vaz and K.J. William, Integrated finite-element analysis of coupled thermoviscoplastic problems, J. Thermal Stresses 4 (1981) 121-153.

[14] M. Ortiz and E.P. Popov. Accuracy and stability of integration algorithms for elastoplastic constitutive relations, Internat. J. Numer. Methods Engrg. 21 (1985) 1561-1576.

[15] T. Belytschko and R. Mullen, Mesh partitions of explicit-implicit time integrations, U.S.-Germany Symposium on Formulations and Computational Algorithms in Finite Element Analysis (MIT Press, Cambridge, MA, 1978).

[16] P. Perzyna, Fundamental problems in viscoplasticity, Adv. Appl. Mech. 9 (1966) 243-377.

[17] S. Ghosh and N. Kikuchi, Finite element formulation for the simulation of hot sheet metal forming processes, Internat. J. Engrg. Sci. 26 (2) (1988) 143-161.

[18] M.L. Wilkins, Calculation of elastic-plastic flow, in: B. Adler et al., eds., Methods of Computational Physics, Vol. 3 (Academic Press, New York, 1964).

[19] R.D. Krieg and D.B. Krieg, Accuracies of numerical solution methods for the elastic-perfectly plastic model, ASME J. Pressure Vessel Tech. 99 (1977) 510-515.

[20] M. Ortiz, P.M. Pinsky and R.L. Taylor, Operator split method for the numerical solution of the elastoplastic dynamic problem, Comput. Methods Appl. Mech. Engrg. 39 (1983) 137-157.

[21] J.R. Rice and D.M. Tracey, Computational fracture mechanics, in: S.J. Fenves, ed., Proc. Symp. Numerical Methods in Structural Mechanics (Academic Press, New York, 1973) 585.

[22] M. Ortiz and J.C. Simo, An analysis of a new class of integration algorithms for elasto-plastic constitutive relations, Internat. J. Numer. Methods Engrg. 23 (1986) 353-366. 
[23] A.E. Green and P.M. Naghdi, A general theory of an elastic-plastic continuum, Arch. Rat. Mech. Anal. 18 (1965) 251-281.

[24] A.E. Green and B.C. McInnis, Generalized hypo-elasticity, Proc. Roy. Soc. Edinburgh A57 (1967) 220.

[25] J.K. Dienes, On the analysis of rotation and stress in deforming bodies, Acta Mech. 32 (1979) 217.

[26] G.C. Johnson and D.J. Bammann, A discussion of stress rates in finite deformation problems, Sandia Report, SAND 82-8821, 1982.

[27] J. Eftis and D.L. Jones, Evaluation and development of constitutive relations for inelastic behavior, Technical Report, Air Force Office of Scientific Research, Washington DC, 1983.

[28] T.J.R. Hughes, Unconditionally stable algorithms for nonlinear heat conduction, Comput. Methods Appl. Mech. Engrg. 10 (1977) 135-139.

[29] T.J.R. Hughes and T. Belytschko, A precis of developments in computational methods for transient analysis, J. Appl. Mech. 50 (1983) 1033-1041.

[30] T.J.R. Hughes, Analysis of transient algorithms with particular reference to stability behaviour, in: T. Belytschko and T.J.R. Hughes, eds., Computational Methods for Transient Analysis, Vol. 1 (North-Holland, Amsterdam, 1983).

[31] T.J.R. Hughes and J. Winget, Finite rotation effects in numerical integration of rate constitutive arising in large-deformation analysis, Internat. J. Numer. Methods Engrg. 15 (12) (1980) 1862-1867.

[32] N. Kikuchi and J.T. Oden, Contact problems in elastostatics, in: J.T. Oden and G.F. Carey, eds., Finite Elements; Special Problems in Solid Mechanics, Vol. V (Prentic-Hall, Englewood Cliffs, NJ, 1984).

[33] N. Kikuchi and Y.J. Song, Penalty/finite element approximations of a class of unilateral problems in linear elasticity, Quart. Appl. Math. XXXIX (1981) 1-22.

[34] P.M. Pinsky, M. Ortiz and K.S. Pister, Numerical integration of rate constitutive equations in finite deformation analysis, Comput. Methods Appl. Mech. Engrg. 40 (1983) 137-158.

[35] T.J.R. Hughes, Numerical implementation of constitutive models: rate-independent deviatoric plasticity, in: S. Nemat-Nasser, R.J. Asaro and G.A. Hegemier, eds., Theoretical Foundations for Large Computations of Nonlinear Behavior (Martinus Nijhoff, Dordrecht, 1984).

[36] K.J. Bathe and A.P. Cimento, Some practical procedures for the solution of nonlinear finite element equations, Comput. Methods Appl. Mech. Engrg. 22 (1980) 59-85.

[37] H. Matthies and G. Strang. The solution of nonlinear finite element equations, Internat. J. Numer. Methods Engrg. 14 (11) (1979) 1613-1626.

[38] H.F. Walker, Numerical solutions of nonlinear equation, University of California, Lawrence Livermore National Laboratory, Rept. UCID-18285, 1979.

[39] J.O. Hallquist, Nike 2D: An implicit, finite deformation, finite element code for analyzing the static and dynamic response of two-dimensional solids, University of California, Lawrence Livermore Laboratory, Rept. UCRL-52678.

[40] T.J.R. Hughes, Generalization of selective integration procedures to anisotropic and nonlinear media, Internat. J. Numer. Methods Engrg. 15 (1980) 1413-1418.

[41] T. Belytschko and W.E. Bachrach, Simple quadrilaterals with high coarse mesh accuracy, AMD-73 (1985) 39-56.

[42] B.C. Koh and N. Kikuchi, New improved hourglass control for bilinear and trilinear elements in anisotropic linear elasticity, Comput. Methods Appl. Mech. Engrg. 65 (1987) 1-46.

[43] D.R.J. Owen and E. Hinton, Finite elements in Plasticity; Theory and practice (Pineridge, Swansea, 1980).

[44] S.W. Key, On an implementation of finite strain plasticity in transient dynamic large-deformation calculations, in: S. Nemat-Nasser, R.J. Asaro and G.A. Hegemier, eds. (Martinus Nijhoff, Dordrecht, 1984).

[45] G. Dumas and C.N. Baronet, Elastoplastic indentation of a half-space by an infinitely long rigid circular cylinder, Internat. J. Mech. Sci. 13 (1971) 519-530.

[46] C.J.M. Gelten and A.W.A. Konter, Application of mesh rezoning in the updated Lagrangian method to metal forming analyses, in: J.F.T. Pittman, R.D. Wood, J.M. Alexander and O.C. Zienkiewicz, eds., Numerical Methods in Industrial Forming Processes (Pineridge, Swansea, 1982). 